\title{
Reversible C-C Double Bond Cleavage to Form a Metal Carbene and an Alkene Enabled on an Iridium Complex Bearing a Pincer-type Alkoxycarbene Ligand
}

Kohei Takahashi $^{a}$ and Nobuharu Iwasawa ${ }^{a *}$

${ }^{a}$ Department of Chemistry, School of Science, Tokyo Institute of Technology, 2-12-1, O-okayama, Meguro-ku, Tokyo 152-8551, Japan

Table of contents

Synthesis of $\mathrm{S1}$ S2

Synthesis of $\mathbf{S 2}$ S2

Synthesis of $\mathrm{S3}$ S3

Synthesis of $L$ S4

Assignment of ${ }^{1} \mathrm{H}$ and ${ }^{13} \mathrm{C}$ NMR signals (signals of $\mathrm{BAr}{ }^{\mathrm{F}}$ were omitted) of 56 Ir_C2_alkene

Assignment of ${ }^{1} \mathrm{H}$ and ${ }^{13} \mathrm{C}$ NMR signals (signals of $\mathrm{BAr}^{\mathrm{F}}$ were omitted) of S7 Ir_C 8_ally

Assignment of ${ }^{1} \mathrm{H}$ and ${ }^{13} \mathrm{C}$ NMR signals (signals of $\mathrm{BAr}{ }^{\mathrm{F}}$ were omitted) of S8 Ir_C8_alkene

Comparison of ${ }^{1} \mathrm{H}$ and ${ }^{13} \mathrm{C}$ NMR chemical shifts of Ir- $\mathrm{C}_{2}$ alkene and Ir- $\mathrm{C}_{8}$ alkene S9 Assignment of ${ }^{1} \mathrm{H}$ and ${ }^{13} \mathrm{C}$ NMR signals (signals of $\mathrm{BAr}^{\mathrm{F}}$ were omitted) of S11 Ir'_C2_alkene

Assignment of ${ }^{1} \mathrm{H}$ and ${ }^{13} \mathrm{C}$ NMR signals (signals of $\mathrm{BAr}{ }^{\mathrm{F}}$ were omitted) of Ir'_C 2_allyI

Table S13. Crystal data and structure refinement for Ir-CI, Ir-CO and Ir- $\mathrm{C}_{2}$ alkene DFT analysis

Observation of the regeneration of Ir' $-C_{2}$ alkene from Ir' $-C_{2}$ allyl under CO NMR spectra 


\section{Synthesis of S1}

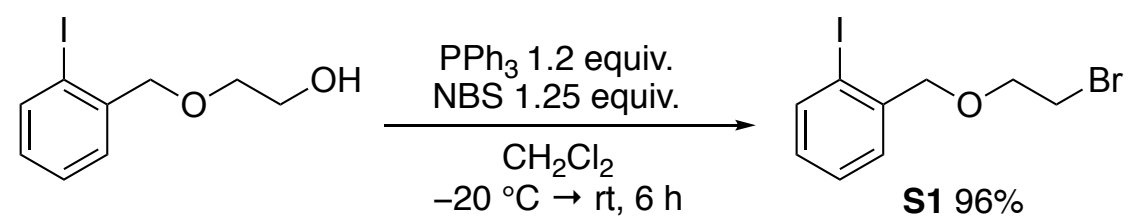

To a $50 \mathrm{~mL}$ two-neck round-bottomed flask charged with 2-(2-iodobenzyloxy)ethanol ${ }^{1}$ (1.15 $\mathrm{g}, 4.12$ mmol), $\mathrm{PPh}_{3}(1.30 \mathrm{~g}, 4.96 \mathrm{mmol})$ and $\mathrm{CH}_{2} \mathrm{Cl}_{2}(15 \mathrm{~mL})$ was added NBS $(0.917 \mathrm{~g}, 5.15 \mathrm{mmol})$ portionwise at $-20{ }^{\circ} \mathrm{C}$. The mixture was slowly warmed up to room temperature and stirred at room temperature for 6 h. Water was added to the mixture, which was extracted three times with $\mathrm{CH}_{2} \mathrm{Cl}_{2}$, and the combined organic extracts were washed with water, brine, and dried over $\mathrm{MgSO}_{4}$. After evaporation of the solvent, the resulting oil was purified by passing through a short pad of silica gel (eluted with Hexane : EtOAc $=$ $4: 1)$ to give the desired compound as a yellow oil (1.35 g, $3.95 \mathrm{mmol}, 96 \%$ yield).

${ }^{1} \mathrm{H}$ NMR $\left.\left(\mathrm{CDCl}_{3}, 500 \mathrm{MHz}\right): \delta=7.83(\mathrm{~d}, J=7.5 \mathrm{~Hz}, 1 \mathrm{H})\right), 7.47,(\mathrm{~d}, J=7.5 \mathrm{~Hz}, 1 \mathrm{H}), 7.46(\mathrm{t}, J=7.5 \mathrm{~Hz}$, $1 \mathrm{H}), 7.00(\mathrm{t}, J=7.5 \mathrm{~Hz}, 1 \mathrm{H}), 4.58(\mathrm{~s}, 2 \mathrm{H}), 3.88(\mathrm{t}, J=6.0 \mathrm{~Hz}, 2 \mathrm{H}), 3.54(\mathrm{t}, J=6.0 \mathrm{~Hz}, 2 \mathrm{H})$.

${ }^{13} \mathrm{C} \mathrm{NMR}\left(\mathrm{CDCl}_{3}, 125 \mathrm{MHz}\right): \delta=139.9,139.1,129.3,128.7,128.2,97.7,76.6,70.4,30.2$.

Anal. Calcd for $\mathrm{C}_{9} \mathrm{H}_{10}$ BrIO: C, 31.70; H, 2.96; Found: C, 31.69; H, 2.99.

IR (ATR): $\mathrm{cm}^{-1}:$ 2935, 2857, 2052, 2037, 2000, 1584, 1560, 1459, 1435, 1422, 1353, 1273, 1198, 1101, $1090,1042,1010,982,947,867,829,811,746$.

\section{Synthesis of S2}

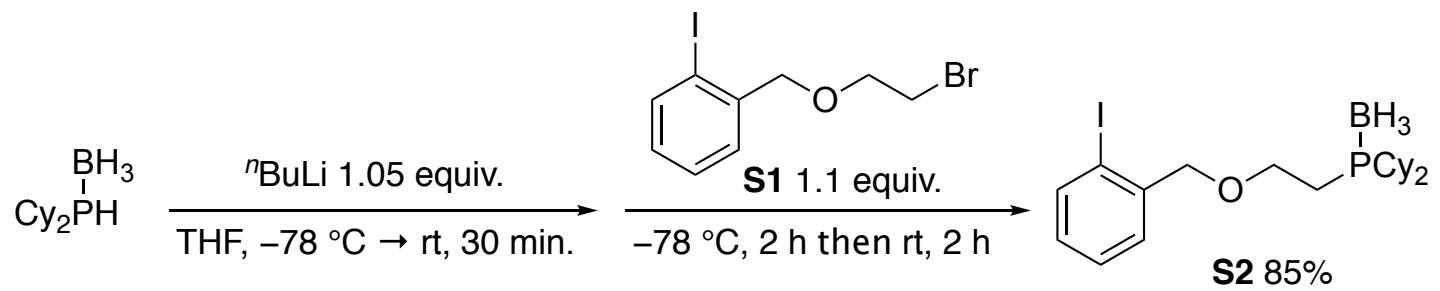

To a $500 \mathrm{~mL}$ two-neck round-bottomed flask charged with dicyclohexylphosphine-borane ${ }^{2}(8.53 \mathrm{~g}, 29.0$ mmol) and dry THF $(100 \mathrm{~mL})$ was added ${ }^{n} \mathrm{BuLi}(1.6 \mathrm{M}$ in hexane, $19.0 \mathrm{~mL}, 30.4 \mathrm{mmol})$ dropwise at -78 ${ }^{\circ} \mathrm{C}$. The mixture was warmed up to room temperature and stirred for 30 minutes. The solution was cooled to $-78{ }^{\circ} \mathrm{C}$ again, and was added via cannula to a $500 \mathrm{~mL}$ round-bottomed flask charged with $\mathbf{S 1}(10.9 \mathrm{~g}$, $32.0 \mathrm{mmol})$ and $\mathrm{THF}(50 \mathrm{~mL})$ at $-78{ }^{\circ} \mathrm{C}$. The mixture was stirred at $-78{ }^{\circ} \mathrm{C}$ for $2 \mathrm{~h}$, then warmed up to room temperature and stirred for $2 \mathrm{~h}$. Water (c.a. $100 \mathrm{~mL}$ ) and EtOAc (c.a. $100 \mathrm{~mL}$ ) were added to the mixture to give a white suspension. The resulting white solid was collected by filtration, and dissolved in $\mathrm{CH}_{2} \mathrm{Cl}_{2}$, which was washed with brine and dried over $\mathrm{Na}_{2} \mathrm{SO}_{4}$. Evaporation of the solvent gave the pure desired compound $(10.1 \mathrm{~g})$. The filtrate of the initial work-up was transferred to another separatory funnel and extracted twice with EtOAc, which was washed with water and brine, and dried over $\mathrm{Na}_{2} \mathrm{SO}_{4}$. 
After evaporation of the solvent, the resulting solid was washed twice with EtOAc to give the desired compound $(1.5 \mathrm{~g})$ as a white solid. The two fractions of the desired compound were combined to determine the yield (11.6 g, $24.6 \mathrm{mmol}, 85 \%$ yield).

${ }^{1} \mathrm{H}$ NMR $\left(\mathrm{CDCl}_{3}, 500 \mathrm{MHz}\right): \delta=7.83(\mathrm{dd}, J=8.0,1.0 \mathrm{~Hz}, 1 \mathrm{H}), 7.41(\mathrm{dd}, J=8.0,1.5 \mathrm{~Hz}, 1 \mathrm{H}), 7.34(\mathrm{dt}, J$ $=1.0,7.5 \mathrm{~Hz}, 1 \mathrm{H}), 6.99(\mathrm{dt}, J=2.0,7.5 \mathrm{~Hz}, 1 \mathrm{H}), 4.49(\mathrm{~s}, 2 \mathrm{H}), 3.79$ (q, $J=8.0 \mathrm{~Hz}, 2 \mathrm{H}), 2.03-1.65(\mathrm{~m}$, $14 \mathrm{H}), 1.44-1.14(\mathrm{~m}, 10 \mathrm{H}), 0.77--0.20$ (brq, 3H)

${ }^{13} \mathrm{C} \mathrm{NMR}\left(\mathrm{CDCl}_{3}, 125 \mathrm{MHz}\right): \delta=140.2,139.2,129.3,128.9,128.2,98.0,66.1,31.9(\mathrm{~d}, J=32 \mathrm{~Hz})$, 27.2-26.6, 25.9, 19.9, 19.6 (One carbon is missing, probably by overlapped with the signal of $\mathrm{CDCl}_{3}$ ).

${ }^{31} \mathrm{P} \mathrm{NMR}\left(\mathrm{CDCl}_{3}, 158 \mathrm{MHz}\right) \delta=23(\mathrm{brm})$

Anal. Calcd for $\mathrm{C}_{21} \mathrm{H}_{35}$ BIOP: C, 53.42; H, 7.47; Found: C, 53.11; H, 7.45.

HRMS (FD ${ }^{+}$) m/z: $[\mathrm{M}-\mathrm{H}]^{+}$Calcd for $\mathrm{C}_{21} \mathrm{H}_{34} \mathrm{BIOP} 471.14795$; Found 471.14851.

IR (ATR): $\mathrm{cm}^{-1}: 2928,2850,2783,2358,2333,1448,1437,1403,1387,1347,1275,1239,1202,1133$, 1120, 1103, 1060, 1046, 1014, 1006, 993, 984, 917, 887, 865, 854, 828, 818, 803, 793, 785, 751, 688, 658.

\section{Synthesis of S3}

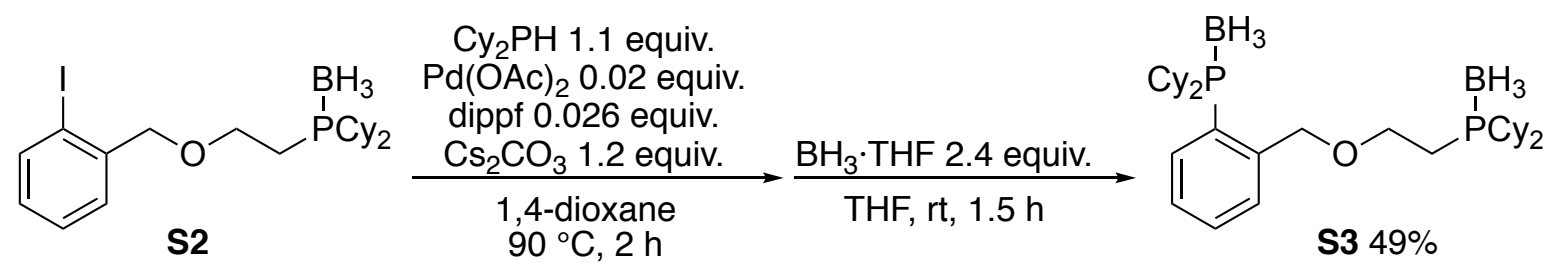

To a $20 \mathrm{~mL}$ Schlenk tube equipped with a J. Young valve was added $\mathbf{S 2}$ (100.3 $\mathrm{mg}, 0.212 \mathrm{mmol}$ ) and $\mathrm{Cs}_{2} \mathrm{CO}_{3}(82.7 \mathrm{mg}, 0.254 \mathrm{mmol})$ under air. Then the flask was brought into an argon-filled glove box, and $\mathrm{Pd}(\mathrm{OAc})_{2}(1.0 \mathrm{mg}, 4.5 \mu \mathrm{mol}$ ), 1,1'-bis(diisopropylphosphino)ferrocene (2.3 $\mathrm{mg}, 5.5 \mu \mathrm{mol})$, dry 1,4-dioxane $(0.2 \mathrm{~mL})$ and dicyclohexylphosphine $(48 \mu \mathrm{L}, 47 \mathrm{mg}, 0.24 \mathrm{mmol})$ were added. The J. Young valve was closed and the mixture was stirred at $90{ }^{\circ} \mathrm{C}$ for $2 \mathrm{~h}$. In the glove box, resulting solids were removed by filtration, washed with THF, and the filtrate was concentrated to c.a. $2 \mathrm{~mL}$ by evaporation of the solvent. To the solution, $\mathrm{BH}_{3} \bullet \mathrm{THF}(0.9 \mathrm{M}, 0.55 \mathrm{~mL}, 0.50 \mathrm{mmol})$ was added and this mixture was stirred at room temperature for $1.5 \mathrm{~h}$. Then the excess $\mathrm{BH}_{3}$ was carefully quenched with water at $0{ }^{\circ} \mathrm{C}$. Resulting mixture was transferred to a separating funnel, and extracted three times with EtOAc. The combined extract was washed with water and brine, and dried over $\mathrm{MgSO}_{4}$. Evaporation of the solvent, and silica gel column chromatography (Hexane : EtOAc $=15: 1$ to $9: 1$ ) gave the desired compound (57.4 mg, $0.103 \mathrm{mmol}, 49 \%$ yield) as a colorless solid.

${ }^{1} \mathrm{H}$ NMR $\left(\mathrm{CDCl}_{3}, 500 \mathrm{MHz}\right): \delta=7.74(\mathrm{t}, J=9.0 \mathrm{~Hz}, 1 \mathrm{H}), 7.59(\mathrm{dd}, J=7.5,2.5 \mathrm{~Hz}, 1 \mathrm{H}), 7.47(\mathrm{t}, J=7.5$ $\mathrm{Hz}, 1 \mathrm{H}), 7.35$ (t, $J=7.5 \mathrm{~Hz}, 1 \mathrm{H}), 4.75$ (s, 2H), 3.80 (q, $J=7.5 \mathrm{~Hz}, 2 \mathrm{H}), 2.15$ (q, $J=12 \mathrm{~Hz}, 2 \mathrm{H}), 2.02-$ 1.57 (m, 22H), $1.54-1.10$ (m, 22H), $1.10--0.10$ (brm, 6H) 
${ }^{13} \mathrm{C} \mathrm{NMR}\left(\mathrm{CDCl}_{3}, 125 \mathrm{MHz}\right): \delta=143.4,134.3,131.0,130.1(\mathrm{~d}, J=8 \mathrm{~Hz}), 127.4(\mathrm{~d}, J=9 \mathrm{~Hz}), 124.3(\mathrm{~d}$, $J=43 \mathrm{~Hz}), 71.1(\mathrm{~d}, J=3 \mathrm{~Hz}), 66.0(\mathrm{~d}, J=3 \mathrm{~Hz}), 34.0(\mathrm{~d}, J=33 \mathrm{~Hz}), 31.8$ (d, $J=33 \mathrm{~Hz}), 27.5,27.2-26.4$ (m), 25.9, 25.8, 19.8, 19.6.

${ }^{31} \mathrm{P} \mathrm{NMR}\left(\mathrm{CDCl}_{3}, 200 \mathrm{MHz}\right): \delta=30$ (brs, 1P), 24.8 (brm, 1P)

Anal. Calcd for $\mathrm{C}_{33} \mathrm{H}_{60} \mathrm{~B}_{2} \mathrm{OP}_{2}$ : C, 71.24; H, 10.87; Found: C, 71.32; H, 10.94 .

HRMS (FD ${ }^{+}$) m/z: [M - H] $]^{+}$Calcd for $\mathrm{C}_{33} \mathrm{H}_{59} \mathrm{~B}_{2} \mathrm{OP}_{2}$ 555.42218; Found 555.42466.

IR (ATR): $\mathrm{cm}^{-1}:$ 2924, 2850, 2365, 2343, 1448, 1355, 1295, 1272, 1204, 1180, 1133, 1118, 1096, 1062, $1003,992,917,887,852,822,798,762,753$.

\section{Synthesis of $L$}

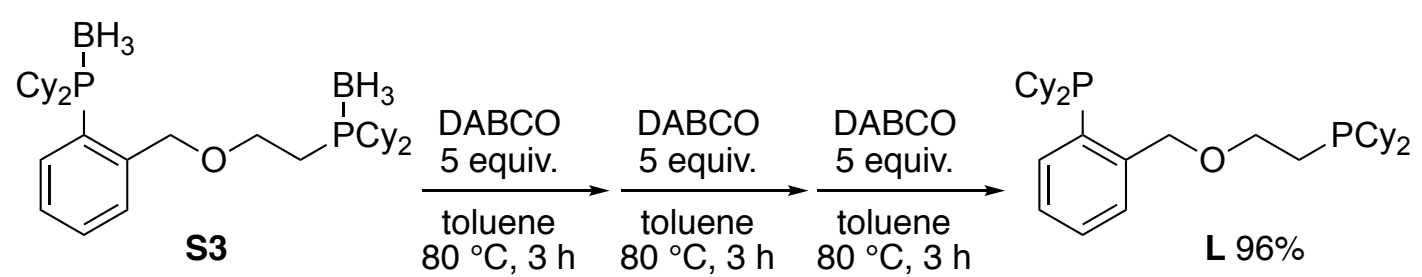

For the complete deprotection of the phosphine-borane, the reaction with DABCO needed to be repeated three times.

To a $20 \mathrm{~mL}$ Schlenk tube equipped with a J. Young valve was added S3 (25.5 mg, $45.8 \mu \mathrm{mol})$, DABCO (25.6 mg, $0.228 \mathrm{mmol}$ ) and toluene $(0.25 \mathrm{~mL})$, and the mixture was degassed by three freeze-pump-thaw cycles and filled with $\mathrm{N}_{2}$. The solution was stirred at $80{ }^{\circ} \mathrm{C}$ for $3 \mathrm{~h}$. In an argon-filled glove box, the solution was passed through a short pad of silica gel $(\varphi 0.5 \mathrm{~cm} \times 4 \mathrm{~cm})$, by eluting with $\mathrm{Et}_{2} \mathrm{O}$ (c.a. $\left.10 \mathrm{~mL}\right)$. After the solvent was removed under vacuum, DABCO $(26.1 \mathrm{mg}, 0.233 \mathrm{mmol})$ and toluene $(0.25 \mathrm{~mL})$ were added to the mixture, and the mixture was stirred at $80^{\circ} \mathrm{C}$ for $3 \mathrm{~h}$. In the argon-filled glove box, the solution was passed through a short pad of silica gel $(\varphi 0.5 \mathrm{~cm} \times 4 \mathrm{~cm})$ by eluting with $\mathrm{Et}_{2} \mathrm{O}$ (c.a. $\left.10 \mathrm{~mL}\right)$. After the solvent was removed under vacuum, DABCO $(25.6 \mathrm{mg}, 0.228 \mathrm{mmol})$ and toluene $(0.25 \mathrm{~mL})$ were added to the mixture, and the mixture was stirred at $80^{\circ} \mathrm{C}$ for $3 \mathrm{~h}$. In the argon-filled glove box, the solution was passed through a short pad of silica gel $(\varphi 0.5 \mathrm{~cm} \times 4 \mathrm{~cm})$ by eluting with $\mathrm{Et}_{2} \mathrm{O}$ (c.a. $5 \mathrm{~mL}$ ). Evaporation of the solvent gave the desired compound (23.3 mg, $44.1 \mu \mathrm{mol}, 96 \%$ yield) as a white solid. ${ }^{1} \mathrm{H}$ NMR $\left(\mathrm{C}_{6} \mathrm{D}_{6}, 500 \mathrm{MHz}\right)$ : 7.88-7.77 (m, 1H), 7.42 (d, $\left.J=7.0 \mathrm{~Hz}, 1 \mathrm{H}\right), 7.25(\mathrm{t}, J=7.5 \mathrm{~Hz}, 1 \mathrm{H}), 7.14$ $\left(1 \mathrm{H}\right.$, overlapped with $\left.\mathrm{C}_{6} \mathrm{HD}_{5}\right), 5.14(\mathrm{~s}, 2 \mathrm{H}), 3.86(\mathrm{q}, J=6.5 \mathrm{~Hz}, 2 \mathrm{H}), 2.10-1.45(\mathrm{~m}, 26 \mathrm{H}), 1.40-1.00(\mathrm{~m}$, 20H).

${ }^{13} \mathrm{C}$ NMR $\left(\mathrm{C}_{6} \mathrm{D}_{6}, 125 \mathrm{MHz}\right): \delta=146.4(\mathrm{~d}, J=25 \mathrm{~Hz}), 134.2(\mathrm{~d}, J=15 \mathrm{~Hz}), 132.6(\mathrm{~d}, J=2 \mathrm{~Hz}), 128.9$, $128.7(\mathrm{~d}, J=6 \mathrm{~Hz}), 126.9,71.7(\mathrm{~d}, J=31 \mathrm{~Hz}), 70.8(\mathrm{~d}, J=34 \mathrm{~Hz}), 34.4(\mathrm{~d}, J=14 \mathrm{~Hz}), 33.7(\mathrm{~d}, J=14$ $\mathrm{Hz}), 31.0(\mathrm{~d}, J=18 \mathrm{~Hz}), 30.6(\mathrm{~d}, J=16 \mathrm{~Hz}), 29.7$ (d, $J=10 \mathrm{~Hz}), 29.2(\mathrm{~d}, J=8 \mathrm{~Hz}), 27.9-27.2(\mathrm{~m}), 26.9$, 26.8, 23.2, 23.1.

${ }^{31} \mathrm{P}$ NMR $\left(\mathrm{C}_{6} \mathrm{D}_{6}, 200 \mathrm{MHz}\right): \quad-9.6(\mathrm{~s}, 1 \mathrm{P}),-15.2(\mathrm{~s}, 1 \mathrm{P})$. 
Anal. Calcd for $\mathrm{C}_{33} \mathrm{H}_{54} \mathrm{OP}_{2}$ : C, 74.96; H, 10.29; Found: C, 74.67; H, 9.96.

HRMS $\left(\mathrm{ESI}^{+}\right) \mathrm{m} / \mathrm{z}:[\mathrm{M}+\mathrm{H}]^{+}$Calcd for $\mathrm{C}_{33} \mathrm{H}_{55} \mathrm{OP}_{2}$ 529.37223; Found 529.3727.

IR (ATR): $\mathrm{cm}^{-1}$; 2919, 2846, 1448, 1346, 1292, 1264, 1238, 1200, 1178, 1167, 1129, 1096, 1077, 1060, 1049, 1001, 958, 911, 885, 848, 816, 742, 723, 714. 


\section{Assignment of ${ }^{1} \mathrm{H}$ and ${ }^{13} \mathrm{C}$ NMR signals (signals of BArF were omitted) of Ir_ $\mathrm{C}_{2}$ alkene}

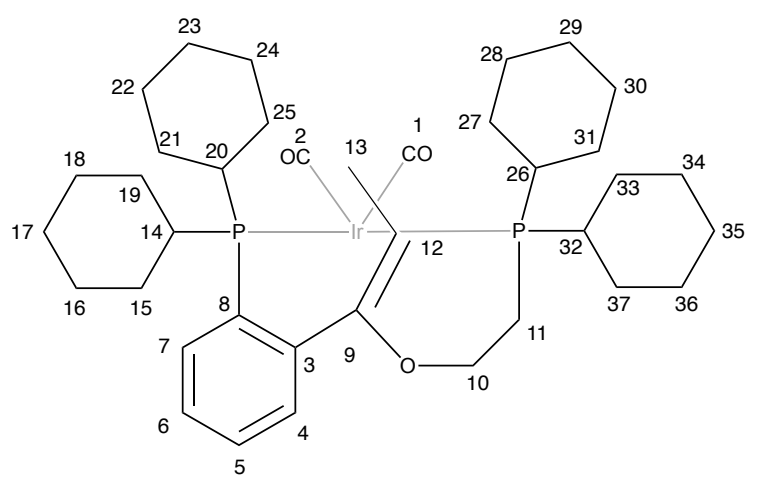

Table S1. Assignment of ${ }^{1} \mathrm{H}$ NMR signals of $\mathbf{I r} \mathbf{C}_{2}$ alkene $\left(\mathrm{CD}_{2} \mathrm{Cl}_{2}\right)$

\begin{tabular}{ll}
\hline Proton & Signals \\
\hline $4-7$ & $7.80-7.65(1 \mathrm{H}), 7.61(1 \mathrm{H}), 7.48(1 \mathrm{H})$, \\
& $7.41(1 \mathrm{H})$. \\
10 & $4.46-4.32(1 \mathrm{H}), 4.02-3.90(1 \mathrm{H})$ \\
12 & $3.42-3.30(1 \mathrm{H})$ \\
13 & $1.14(3 \mathrm{H})$ \\
$11,14-37$, & $2.64-2.50(1 \mathrm{H}), 2.44-1.20(\mathrm{~m}, 43 \mathrm{H})$, \\
& $1.12-0.98(1 \mathrm{H}), 0.44-0.30(1 \mathrm{H})$ \\
\hline
\end{tabular}

Table S2. Assignment of ${ }^{13} \mathrm{C}$ NMR signals of $\mathbf{I r} \_\mathbf{C}_{2}$ alkene

\begin{tabular}{ll}
\hline Carbon & Signals \\
\hline 1,2 & $173.2,172.4$ \\
3 & 151.5 \\
$4-7$ & $133.6,130.5,129.6,127.2$ \\
8 & 123.4 \\
9 & 105.4 \\
10 & 69.3 \\
12 & 40.7 \\
13 & 34.9 \\
$14,20,26,32$ & $40.7,38.9,35.7,34.7$ \\
$11,15-19,21-25,27-31,33-37$ & $30.8-30.6,29.8-25.8,19.0,16.5,16.3$ \\
\hline
\end{tabular}




\section{Assignment of ${ }^{1} \mathrm{H}$ and ${ }^{13} \mathrm{C}$ NMR signals (signals of BAr ${ }^{\mathrm{F}}$ were omitted) of $\mathrm{Ir}_{-} \mathrm{C}_{8_{-} \text {allyl }}$}

Assignments for major $(\alpha)$ or minor $(\beta)$ isomer are indicated if possible.

Proposed two isomers are indicated below.
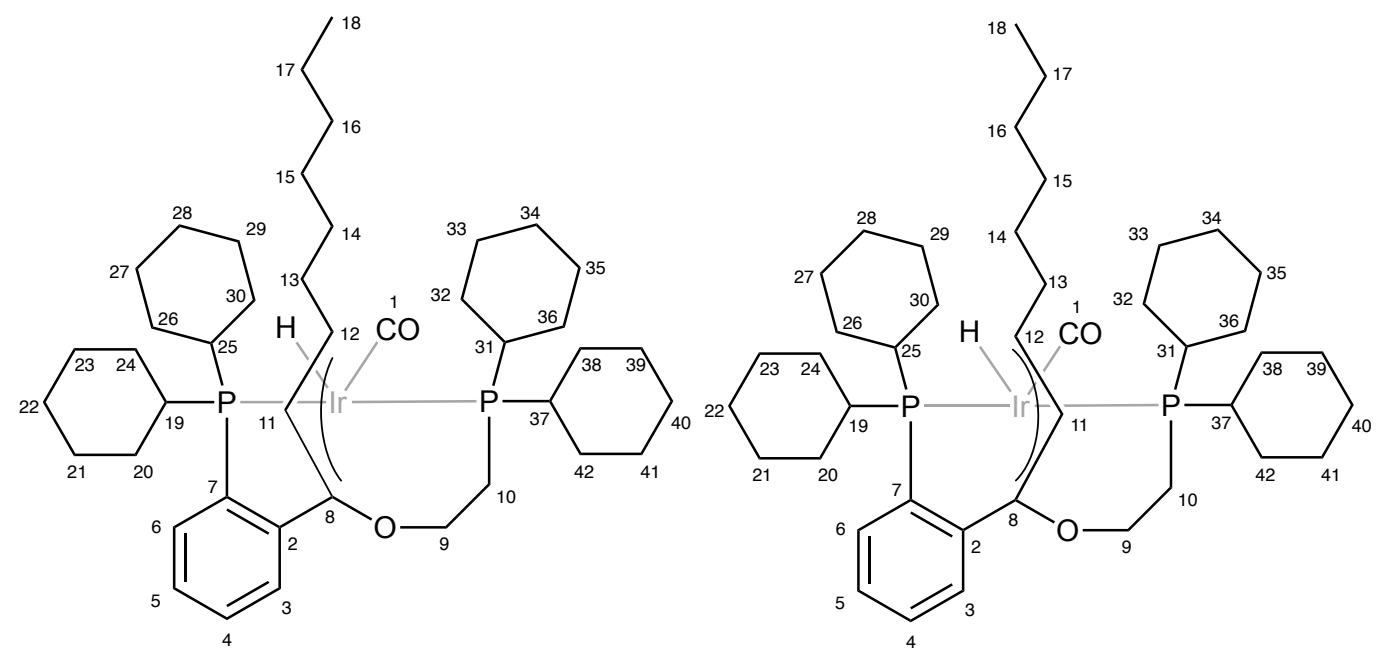

Table S3. Assignment of ${ }^{1} \mathrm{H}$ NMR signals of $\mathbf{I r} \mathbf{C}_{\mathbf{8} \_ \text {allyl }}$

\begin{tabular}{ll}
\hline Proton & Signals \\
\hline $3-6$ & $7.51-7.28(4 \mathrm{H} \alpha$ and $4 \mathrm{H} \beta)$ \\
9 & $4.36-4.13(1 \mathrm{H} \alpha$ and $1 \mathrm{H} \beta), 3.12(1 \mathrm{H} \beta), 2.77(1 \mathrm{H} \alpha)$ \\
11 & $4.97(1 \mathrm{H} \beta), 4.78-4.60(1 \mathrm{H} \alpha)$ \\
12 & $4.78-4.60(1 \mathrm{H} \alpha), 3.46-3.35(1 \mathrm{H} \beta)$ \\
$13-42$ & $2.56(1 \mathrm{H} \beta), 2.45-0.73(59 \mathrm{H} \alpha$ and $58 \mathrm{H} \beta)$ \\
$\mathrm{Ir}-\mathrm{H}$ & $-12.4(1 \mathrm{H} \alpha),-12.7(1 \mathrm{H} \beta)$ \\
\hline
\end{tabular}

Table S4. Assignment of ${ }^{13} \mathrm{C}$ NMR signals of $\mathbf{I r} \_\mathbf{C}_{\mathbf{8} \_ \text {allyl }}$

\begin{tabular}{ll}
\hline carbon & signals \\
\hline 1 & $171.9(\beta), 170.8(\alpha)$ \\
2 & $156.0,149.8$ \\
$3,4,5$ & $133.1,132.3,131.2,129.7,129.6,129.5$ \\
6 & $128.6,124.3$ \\
7 & $126.5,126.1$ \\
8 & $99.4(\beta), 91.9(\alpha)$ \\
9 & $74.7(\beta), 71.4(\alpha)$ \\
11 & $90.8(\alpha), 90.3(\beta)$ \\
12 & $81.0(\beta), 78.9(\alpha)$ \\
$10,13-17,20-24,26-30,32-36,38-42$ & $33.7-25.0,23.1-22.2,17.1,16.9$ \\
$19,25,31,37$ & $42.5,41.2,40.6,39.6,38.2-37.5,36.4$ \\
18 & $13.9,13.8$ \\
\hline
\end{tabular}




\section{Assignment of ${ }^{1} \mathrm{H}$ and ${ }^{13} \mathrm{C}$ NMR signals (signals of BArF were omitted) of Ir_ $\mathrm{C}_{8}$ alkene}

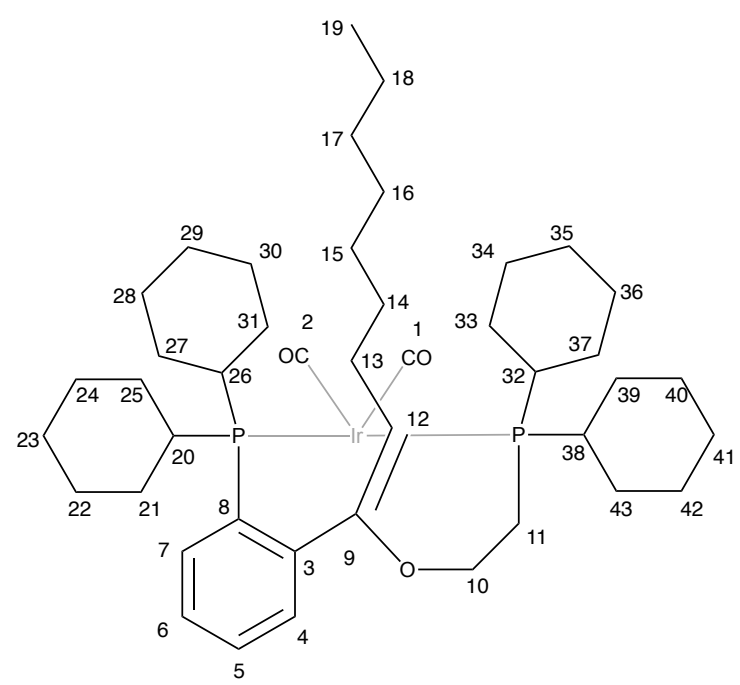

Table S5. Assignment of ${ }^{1} \mathrm{H}$ NMR signals of $\mathbf{I r} \mathbf{C}_{\boldsymbol{8}_{\text {alkene }}}$

\begin{tabular}{ll}
\hline Proton & Signals \\
\hline $4-7$ & $7.57(1 \mathrm{H}), 7.02(1 \mathrm{H}), 6.86(1 \mathrm{H})$ and $1 \mathrm{H}$ \\
& overlapped with $\mathrm{C}_{6} \mathrm{H} \mathrm{D}_{5}$. \\
10 & $3.86-3.70(1 \mathrm{H}), 3.44-3.22(1 \mathrm{H})$ \\
12 & $3.01-2.82(1 \mathrm{H})$ \\
$11,13-43$ & $2.50-0.30(\mathrm{~m}, 60 \mathrm{H}), 0.21-0.00(1 \mathrm{H})$ \\
\hline
\end{tabular}

Table S6. Assignment of ${ }^{13} \mathrm{C}$ NMR signals of $\mathbf{I r}_{-} \mathbf{C}_{\mathbf{8}}$ alkene

\begin{tabular}{ll}
\hline Carbon & Signals \\
\hline 1,2 & $172.9-172.5,172.4-172.2$ \\
3 & 151.4 \\
$4-7$ & $133.0,130.1,129.2,127.1$ \\
8 & 122.9 \\
9 & 104.0 \\
10 & 69.4 \\
12 & 41.4 \\
$20,26,32,38$ & $40.7,38.8,36.5-35.8,34.3$ \\
$11,13-18,21-25,27-31,33-37,39-43$ & $33.0,32.2-25.0,22.8,15.9,15.6$ \\
19 & 14.1 \\
\hline
\end{tabular}


Table S7. Comparison of ${ }^{1} \mathrm{H}$ NMR signals of $\mathbf{I r} \_\mathbf{C}_{2}$ alkene $\left(\mathrm{C}_{6} \mathrm{D}_{6}\right)$ and $\mathbf{I r} \_\mathbf{C}_{\mathbf{8}}$ alkene $\left(\mathrm{C}_{6} \mathrm{D}_{6} / \mathrm{CDCl}_{3}=9: 1\right)$
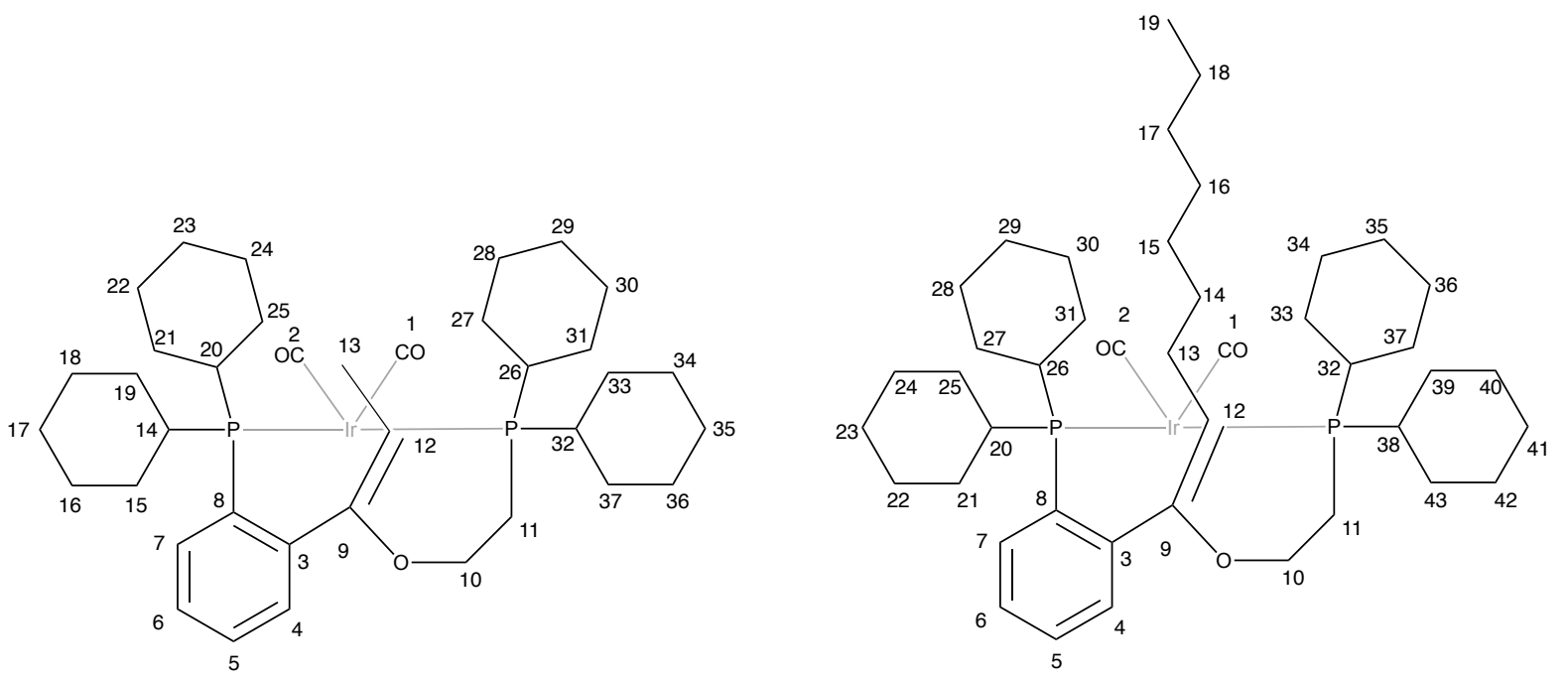

\begin{tabular}{|c|c|c|c|}
\hline Proton & Signals & Proton & Signals \\
\hline $4-7$ & $\begin{array}{l}7.56(1 \mathrm{H}), 6.99(1 \mathrm{H}), 6.73(1 \mathrm{H}) \text { and } 1 \mathrm{H} \\
\text { probably overlapped with } \mathrm{C}_{6} \mathrm{HD}_{5} \text {. }\end{array}$ & $4-7$ & $\begin{array}{l}7.57(1 \mathrm{H}), 7.02(1 \mathrm{H}), 6.86(1 \mathrm{H}) \\
\text { and } 1 \mathrm{H} \text { overlapped with } \mathrm{C}_{6} \mathrm{HD}_{5} .\end{array}$ \\
\hline 10 & 3.75-3.63 (1H), 3.37-3.27 (1H), & 10 & 3.86-3.70 (1H), 3.44-3.22 (1H) \\
\hline 12 & $3.02-2.91(1 \mathrm{H})$ & 12 & $3.01-2.82(1 \mathrm{H})$ \\
\hline 13 & $0.69(3 \mathrm{H})$ & - & - \\
\hline $11,14-37$ & $\begin{array}{lccc}2.10-1.96 & (1 \mathrm{H}), & 1.94-1.84 & (1 \mathrm{H}), \\
2.44-1.20 & (41 \mathrm{H}), & 0.78-0.68 & (1 \mathrm{H}), \\
0.57-0.48(1 \mathrm{H}), 0.16-0.02(1 \mathrm{H}) . & \end{array}$ & $11,13-43$ & $\begin{array}{l}2.50-0.30 \quad(m, \quad 60 H), \quad 0.21-0.00 \\
(1 \mathrm{H})\end{array}$ \\
\hline
\end{tabular}


Table S8. Comparison of ${ }^{13} \mathrm{C}$ NMR signals of $\mathbf{I r} \_\mathbf{C}_{\mathbf{2} \_ \text {alkene }}\left(\mathrm{CD}_{2} \mathrm{Cl}_{2}\right)$ and $\mathbf{I r} \_\mathbf{C}_{\mathbf{8} \_ \text {alkene }}\left(\mathrm{C}_{6} \mathrm{D}_{6} / \mathrm{CDCl}_{3}=9: 1\right)$
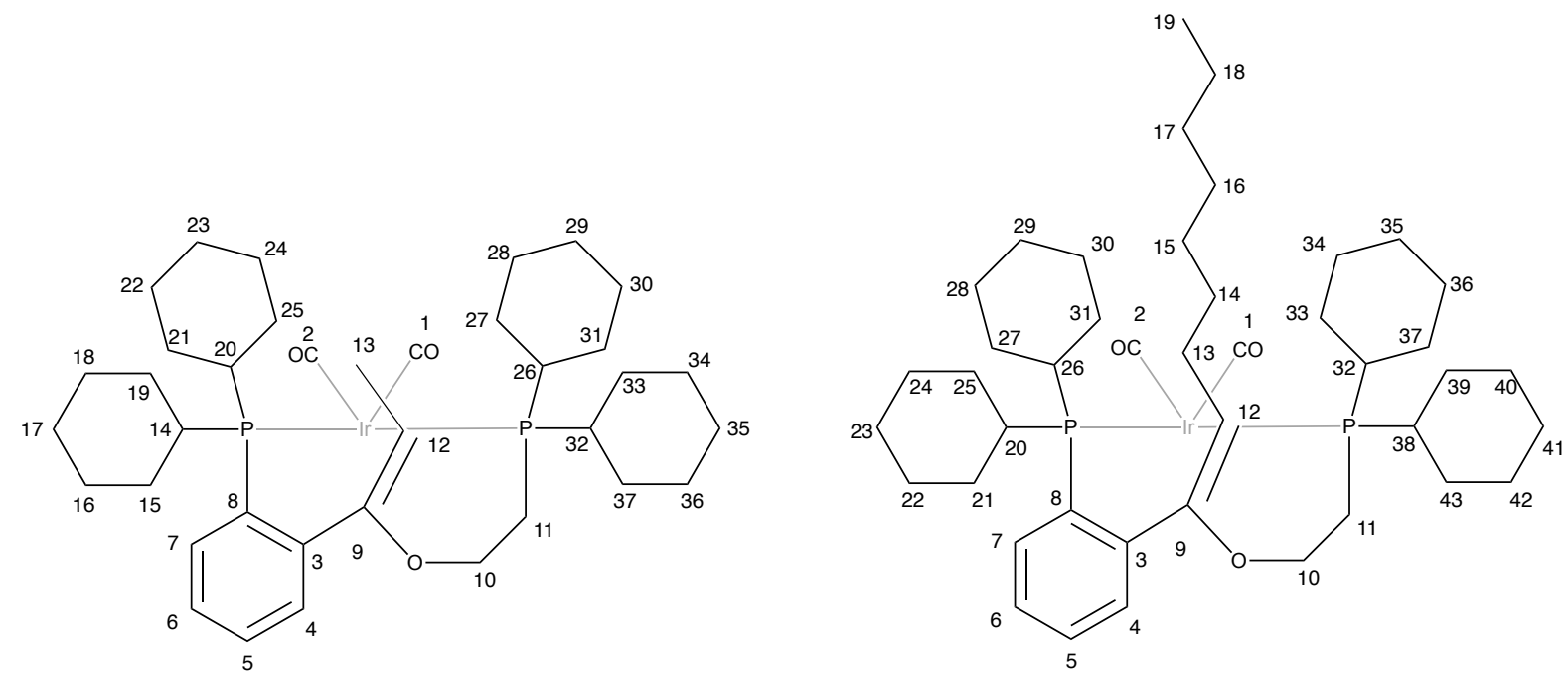

\begin{tabular}{llll}
\hline Carbon & Signals & Carbon & Signals \\
\hline 1,2 & $173.2,172.4$ & 1,2 & $172.9-172.5,172.4-172.2$ \\
3 & 151.5 & 3 & 151.4 \\
$4-7$ & $133.6,130.5,129.6,127.2$ & $4-7$ & $133.0,130.1,129.2,127.1$ \\
8 & 123.4 & 8 & 122.9 \\
9 & 105.4 & 9 & 104.0 \\
10 & 69.3 & 10 & 69.4 \\
12 & 40.7 & 12 & 41.4 \\
$14,20,26,32$ & $40.7,38.9,35.7,34.7$ & $20,26,32,38$ & $40.7,38.8,36.5-35.8,34.3$ \\
$11, \quad 13, \quad 15-19$, & $34.930 .8-30.6,29.8-25.8$, & $11,13-19,21-25$, & $33.0,32.2-25.0,22.8,15.9$, \\
$21-25,27-31,33-37$ & $19.0,16.5,16.3$ & $27-31,33-37,39-43$ & $15.6,14.1$ \\
\hline
\end{tabular}




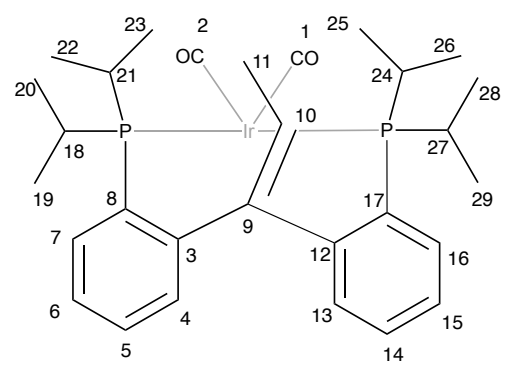

Table S9. Assignment of ${ }^{1} \mathrm{H}$ NMR signals of Ir'_C $\mathbf{C}_{2}$ alkene

\begin{tabular}{ll}
\hline Proton & Signals \\
\hline $4-7,13-16$ & $7.92(1 \mathrm{H}), 7.77(1 \mathrm{H}), 7.65(1 \mathrm{H}), 7.52(1 \mathrm{H})$, \\
& $7.45-7.30(3 \mathrm{H}), 7.23(1 \mathrm{H})$ \\
10 & $3.19-3.07(1 \mathrm{H})$ \\
11 & $1.03(3 \mathrm{H})$ \\
$18,21,24,27$ & $2.90-2.69(2 \mathrm{H}), 2.69-2.56(2 \mathrm{H})$ \\
$19,20,22,23,25,26,28,29$ & $1.54-1.40(6 \mathrm{H}), 1.36-1.22(6 \mathrm{H}), 0.98-0.78$ \\
& $(12 \mathrm{H})$ \\
\hline
\end{tabular}

Table S10. Assignment of ${ }^{13} \mathrm{C}$ NMR signals of Ir'_C $\mathbf{2}_{2}$ alkene

\begin{tabular}{ll}
\hline Carbon & Signals \\
\hline 1,2 & $171.1,170.4$ \\
3,12 & $157.4,146.6$ \\
$4,5,6,7,13,14,15,16$ & $133.6,132.9,131.7,131.0,130.9,128.8,127.1,123.8$ \\
8,17 & $128.6,123.9$ \\
9 & 80.3 \\
10 & 56.3 \\
11 & 20.8 \\
$18,21,24,27$ & $29.1,28.2,27.7,22.8$ \\
$19,20,22,23,25,26,28,29$ & $20.5,20.0,18.1,17.44,17.40,17.3,16.42,16.38$ \\
\hline
\end{tabular}




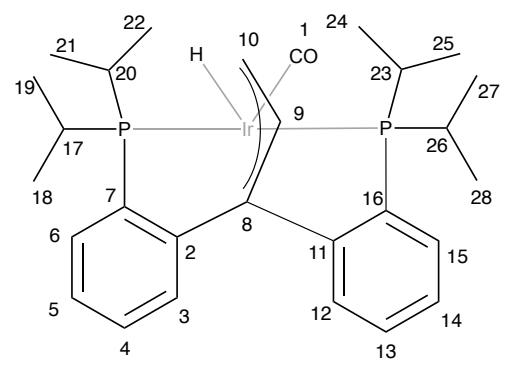

Table S11. Assignment of ${ }^{1} \mathrm{H}$ NMR signals of Ir'_ $\mathbf{C}_{2}$ allyl

\begin{tabular}{ll}
\hline Proton & Signals \\
\hline $3-6,12-15$ & $7.80-7.64(2 \mathrm{H}), 7.57-7.49(1 \mathrm{H}), 7.49-7.40(2 \mathrm{H})$, \\
& $7.36-7.27(3 \mathrm{H})$ \\
9 & $5.32-5.20(1 \mathrm{H})$ \\
10 & $3.85(1 \mathrm{H}), 2.65-2.42(1 \mathrm{H})$ \\
$17,20,23,26$ & $2.86-2.65(2 \mathrm{H}), 2.65-2.42(2 \mathrm{H})$ \\
$18,19,21,22,24,25,27,28$ & $1.51-1.35(9 \mathrm{H}), 1.20-1.07(6 \mathrm{H}), 1.03(3 \mathrm{H}), 0.82$ \\
$\mathrm{Ir}-\mathrm{H}$ & $(3 \mathrm{H}), 0.51(3 \mathrm{H})$ \\
\hline
\end{tabular}

Table S12. Assignment of ${ }^{13}$ C NMR signals of Ir'_C $\mathbf{C}_{2}$ allyl

\begin{tabular}{ll}
\hline Carbon & Signals \\
\hline 1 & $169.5-169.3$ \\
2,11 & $154.3,142.7$ \\
$3,4,5,6,12,13,14,15$ & $133.4,133.2,132.2,131.6,131.3,129.2$, \\
& $127.8,124.4$ \\
7,16 & $133.3,130.7$ \\
8 & 76.2 \\
9 & 98.5 \\
10 & 50.2 \\
$17,20,23,26$ & $32.2,30.8,26.6,25.8$ \\
$18,19,21,22,24,25,27,28$ & $22.6,20.0,19.8,18.5,18.2,17.6,17.24,17.21$ \\
\hline
\end{tabular}


Table S13. Crystal data and structure refinement for Ir-CI, Ir-CO and Ir- $\mathrm{C}_{2}$ alkene

\begin{tabular}{|c|c|c|c|}
\hline & Ir-Cl & Ir-CO $\bullet(1,4 \text {-dioxane })_{3}$ & Ir-C 2_alkene \\
\hline Empirical formula & $\mathrm{C}_{33} \mathrm{H}_{52} \mathrm{ClIrOP}_{2}$ & $\mathrm{C}_{79} \mathrm{H}_{88} \mathrm{BF}_{24} \mathrm{IrO}_{9} \mathrm{P}_{2}$ & $\mathrm{C}_{69} \mathrm{H}_{68} \mathrm{BF}_{24} \mathrm{IrO}_{3} \mathrm{P}_{2}$ \\
\hline Formula weight & 754.33 & 1902.44 & 1666.18 \\
\hline Temperature (K) & 123 & 123.15 & 123 \\
\hline Wavelength $(\AA)$ & 0.71075 & 0.71075 & 0.71075 \\
\hline Crystal system & monoclinic & triclinic & monoclinic \\
\hline Space group & $\mathrm{P} 2{ }_{1} / \mathrm{n}$ & $\mathrm{P}-1$ & $\mathrm{Cc}$ \\
\hline \multirow[t]{6}{*}{ Unit cell dimensions } & $a=12.02260(5) \AA$ & $a=13.3628(13) \AA$ & $a=13.263(2) \AA$ \\
\hline & $b=17.18933(7) \AA$ & $b=17.0291(17) \AA$ & $b=22.443(4) \AA$ \\
\hline & $c=16.93699(7) \AA$ & $c=18.6446(19) \AA$ & $c=25.725(4) \AA$ \\
\hline & $\alpha=90^{\circ}$ & $\alpha=101.5062(14)^{\circ}$ & $\alpha=90^{\circ}$ \\
\hline & $\beta=97.8457(4)^{\circ}$ & $\beta=101.2726(13)^{\circ}$ & $\beta=96.790(3)^{\circ}$ \\
\hline & $\gamma=90^{\circ}$ & $\gamma=96.3572(13)^{\circ}$ & $\gamma=90^{\circ}$ \\
\hline Volume $\left(\AA^{3}\right)$ & $3467.43(2)$ & $4027.4(7)$ & $7604(2)$ \\
\hline$Z$ & 4 & 2 & 4 \\
\hline Density (calculated) & 1.445 & 1.569 & 1.455 \\
\hline Absorption coefficient & 9.195 & 1.809 & 1.899 \\
\hline$F(000)$ & 1528.0 & 1924.0 & 3336.0 \\
\hline Crystal size $\left(\mathrm{mm}^{3}\right)$ & $0.138 \times 0.129 \times 0.059$ & $0.18 \times 0.09 \times 0.07$ & $0.04 \times 0.04 \times 0.03$ \\
\hline $2 \theta$ range for data collection & $7.362^{\circ}$ to $136.502^{\circ}$ & $6.006^{\circ}$ to $54.966^{\circ}$ & $6.006^{\circ}$ to $54.972^{\circ}$ \\
\hline \multirow[t]{2}{*}{ Index ranges } & $-14 \leq \mathrm{h} \leq 13,-20 \leq \mathrm{k} \leq$ & $-17 \leq \mathrm{h} \leq 17,-22 \leq \mathrm{k} \leq$ & $-17 \leq \mathrm{h} \leq 17,-29 \leq \mathrm{k} \leq$ \\
\hline & $20,-20 \leq 1 \leq 20$ & $21,-24 \leq 1 \leq 24$ & $29,-33 \leq 1 \leq 33$ \\
\hline Reflections collected & 45078 & 50148 & 47004 \\
\hline Independent reflections & 6357 & 18380 & 16996 \\
\hline Data / restraints / parameters & $6357 / 0 / 344$ & $18380 / 0 / 1045$ & $16996 / 2 / 938$ \\
\hline Goodness-of-fit on $F^{2}$ & 1.071 & 1.081 & 1.059 \\
\hline \multirow[t]{2}{*}{ Final $R$ indices $[I>2 \operatorname{sigma}(I)]$} & $\mathrm{R}_{1}=0.0161$ & $\mathrm{R}_{1}=0.0438$ & $\mathrm{R}_{1}=0.0686$ \\
\hline & $\mathrm{wR}_{2}=0.10387$ & $\mathrm{wR}_{2}=0.0972$ & $\mathrm{wR}_{2}=0.1605$ \\
\hline \multirow[t]{2}{*}{ Final $R$ indices (all data) } & $\mathrm{R}_{1}=0.0163$ & $\mathrm{R}_{1}=0.0498$ & $\mathrm{R}_{1}=0.0900$ \\
\hline & $\mathrm{wR}_{2}=0.0389$ & $\mathrm{wR}_{2}=0.1035$ & $\mathrm{wR}_{2}=0.1802$ \\
\hline Largest diff. peak and hole (e $\left.\AA^{-3}\right)$ & $0.40 /-0.34$ & $3.41 /-1.22$ & $2.58 /-1.73$ \\
\hline Flack parameter & - & - & $0.109(10)$ \\
\hline
\end{tabular}




\section{DFT analysis}

Geometrical optimizations, frequency and population calculations in the gas-phase were performed by density functional theory (DFT) with Gaussian 16 program, ${ }^{3}$ by using B3LYP ${ }^{4}$ functional with

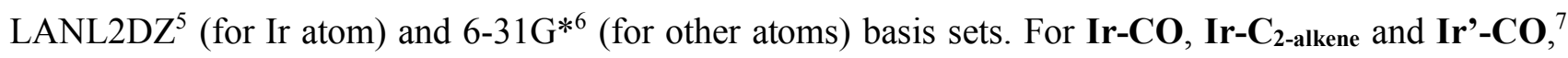
initial structures were extracted from the X-ray structure. The initial structure of Ir'-C $\mathbf{2}_{2}$ alkene was constructed by modifying the calculated structure of $\mathbf{I r}-\mathbf{C}_{\mathbf{2} \_ \text {alkene. }}$.

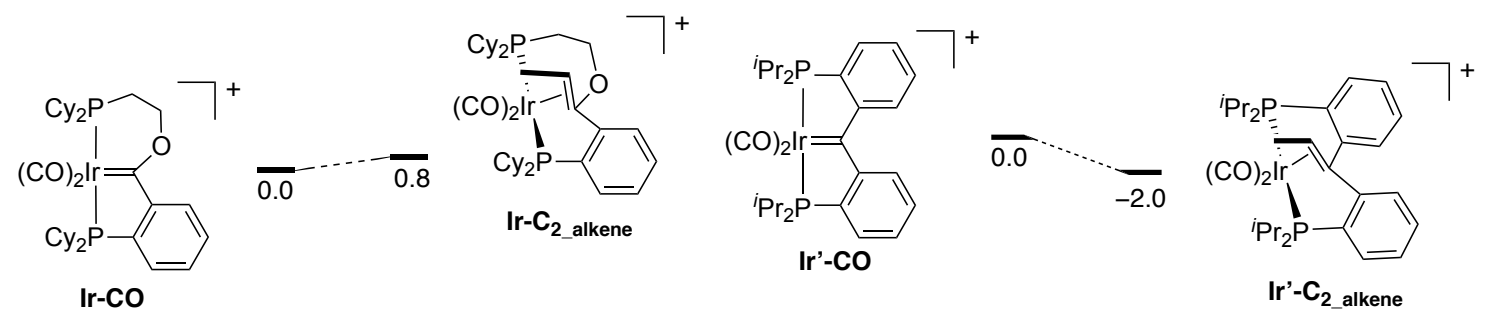

Figure S1. Summary of relative free energy estimated by DFT calculation (kcal/mol)

Similar calculation was also performed on the complexes bearing Me group on the $\mathrm{P}$ atoms instead of Cy or ${ }^{i} \operatorname{Pr}$ groups (Figure S2). In both cases, the reactions become more exothermic compared to those of Cy and ${ }^{i} \operatorname{Pr}$ groups, respectively. Thus, steric hinderance induced by the substituents on the $\mathrm{P}$ atoms was suggested to drive the reaction to Ir/Ir'-CO. However, the difference between Me vs Cy or ${ }^{i} \operatorname{Pr}$ groups were more significant in the case of Ir' system $(-8.6 \mathrm{kcal} / \mathrm{mol})$ than $\mathbf{I r}$ system $(-4.8 \mathrm{kcal} / \mathrm{mol})$. Therefore, steric hinderance does not explain the reason why reverse reaction did not take place in the case of Ir'/Ir'-C 2_allyl. $^{\prime}$

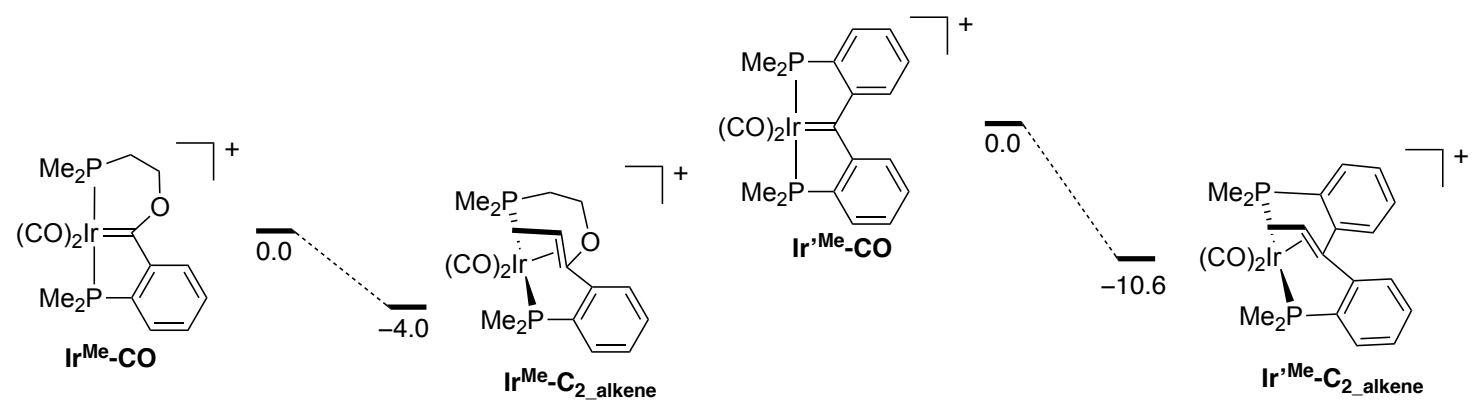

Figure S2. Summary of relative free energy estimated by DFT calculation $(\mathrm{kcal} / \mathrm{mol})$ 


\section{Observation of the regeneration of Ir'- $\mathbf{C}_{2_{-} \text {alkene }}$ from Ir'- $\mathbf{C}_{\mathbf{z}_{-} \text {allyl }}$ under $\mathrm{CO}$}

In order to get information about relative thermodynamic stability of $\mathbf{I r} \mathbf{P}^{-} \mathbf{C}_{\mathbf{2}_{-} \text {alkene }}$ and $\mathbf{I r} \mathbf{r}_{\mathbf{C}} \mathbf{C}_{\mathbf{2}}$ allyl at room temperature, following experiment was conducted.

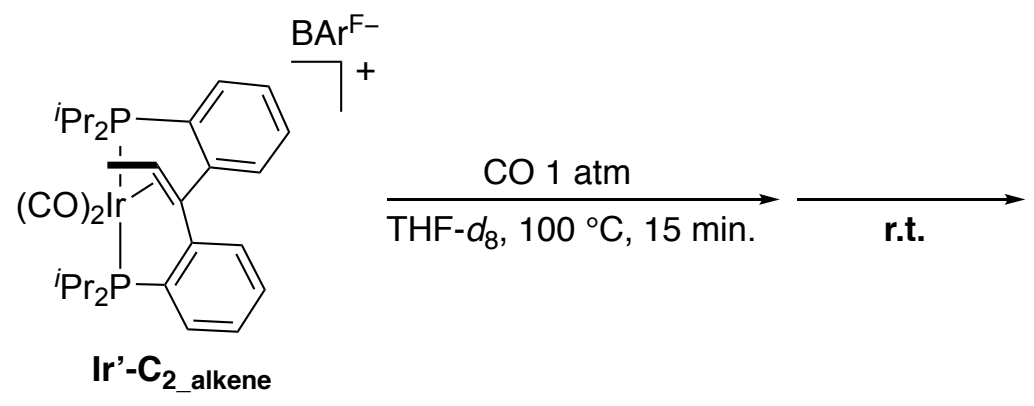

To an NMR tube equipped with a J. Young valve was added Ir'-C $\mathbf{C}_{-}$alkene $(2.3 \mathrm{mg}, 1.5 \mu \mathrm{mol}), \mathrm{THF}-d_{8}(0.5$ $\mathrm{mL})$ and 1,3,5-trimethoxybenzene $(2.0 \mathrm{mg}, 12 \mu \mathrm{mol})$ as an internal standard. To the solution was introduced an atmospheric pressure of carbon monoxide by a freeze-pump-thaw cycle. The resulting solution was analyzed by ${ }^{1} \mathrm{H}$ NMR to determine the molar ratio of $\mathbf{I r}$ '- $\mathbf{C}_{\mathbf{2}_{-} \text {alkene }}$ and the internal standard. The NMR tube was heated at $100{ }^{\circ} \mathrm{C}$ for 15 minutes to give a mixture of $\mathbf{I r}$ '- $\mathbf{C}_{\mathbf{2}}$ alkene and $\mathbf{I r}$ '- $\mathbf{C}_{2} \mathbf{z}_{\text {allyl }}$

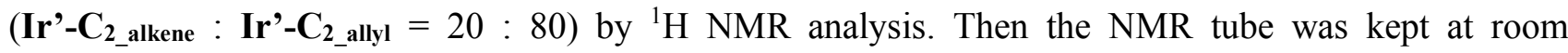
temperature and the reaction progress was monitored by ${ }^{1} \mathrm{H}$ and ${ }^{31} \mathrm{P}$ NMR. The ratio of Ir'- $\mathbf{C}_{2}$ alkene was

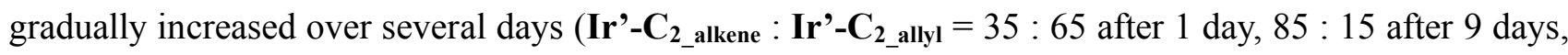
and 95 : 5 after 16 days, ). The yield (recovery) of $\mathbf{I r}$ '- $\mathbf{C}_{2}$ alkene after 16 days was $95 \%$ based on the internal standard. 


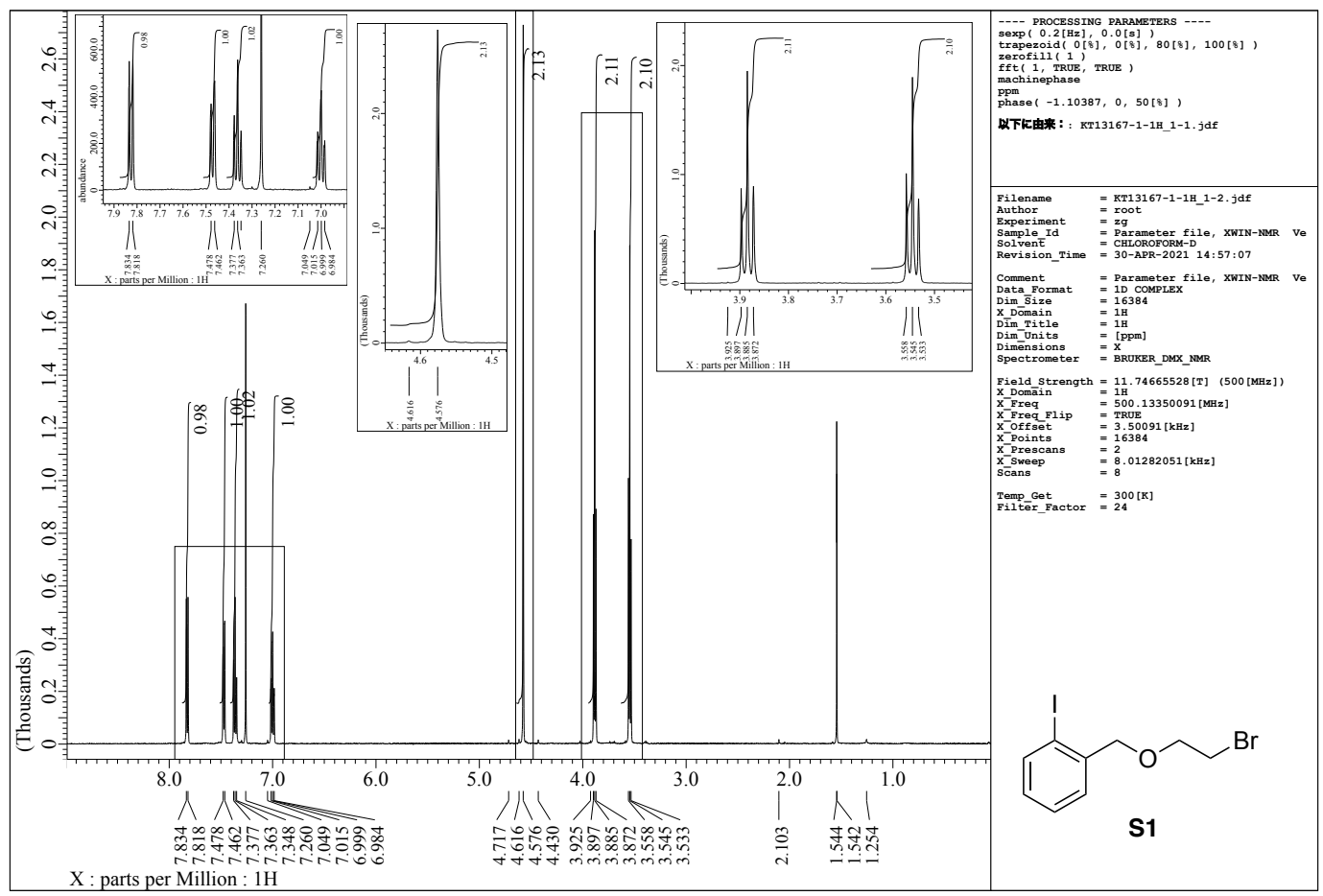

Figure S3. ${ }^{1} \mathrm{H}$ NMR spectrum of $\mathbf{S 1}$

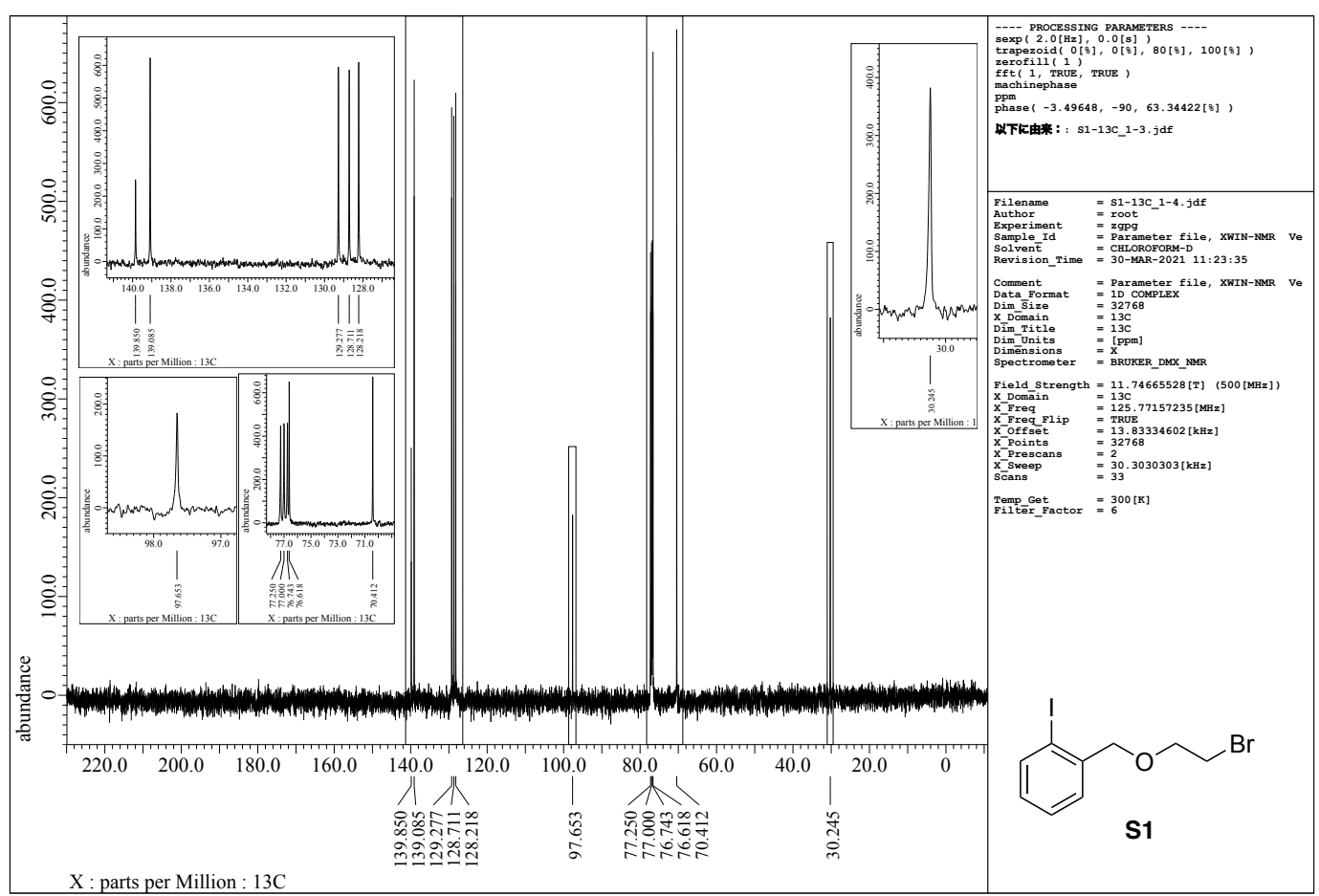

Figure S4. ${ }^{13} \mathrm{C}$ NMR spectrum of S1 


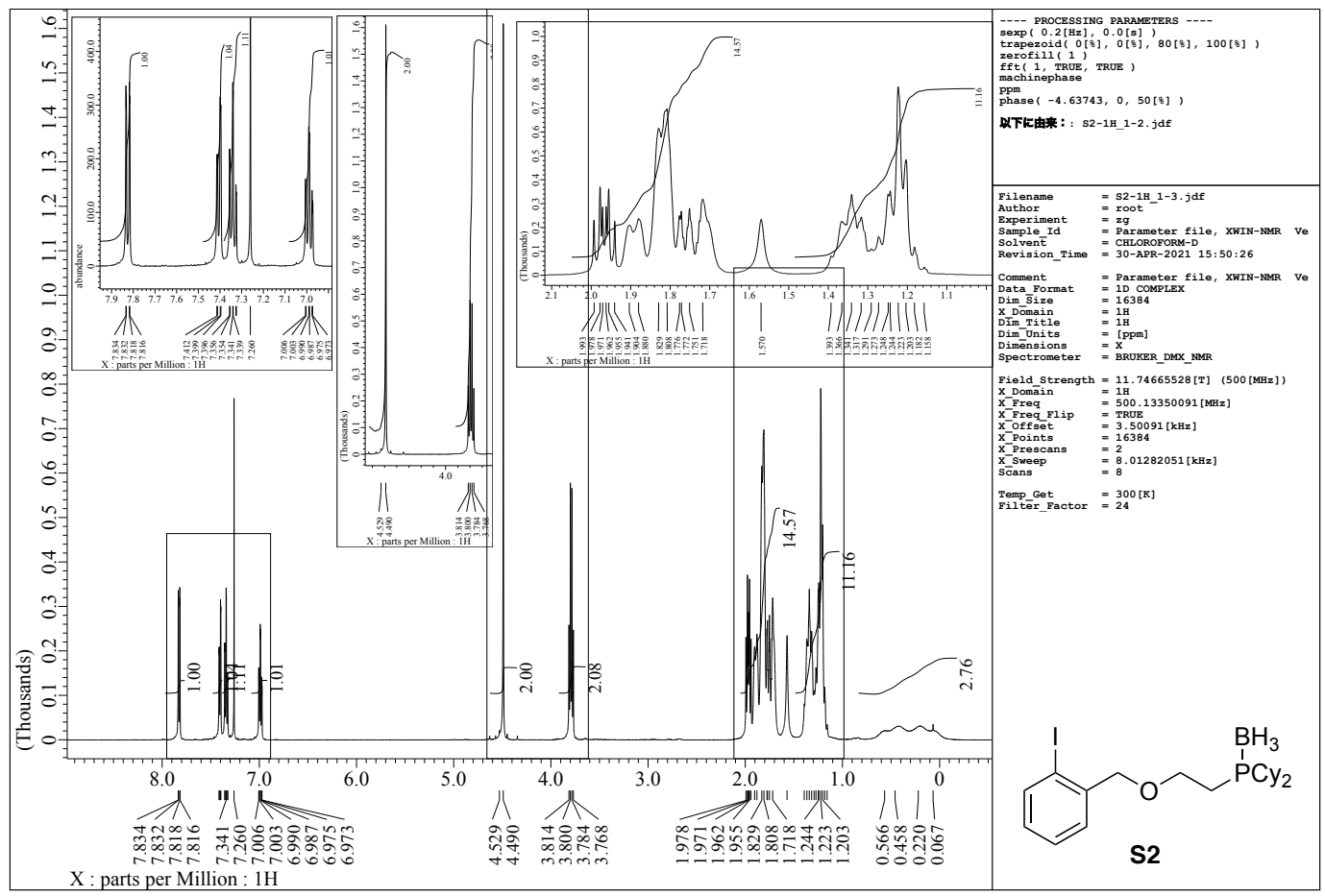

Figure S5. ${ }^{1} \mathrm{H}$ NMR spectrum of $\mathbf{S 2}$

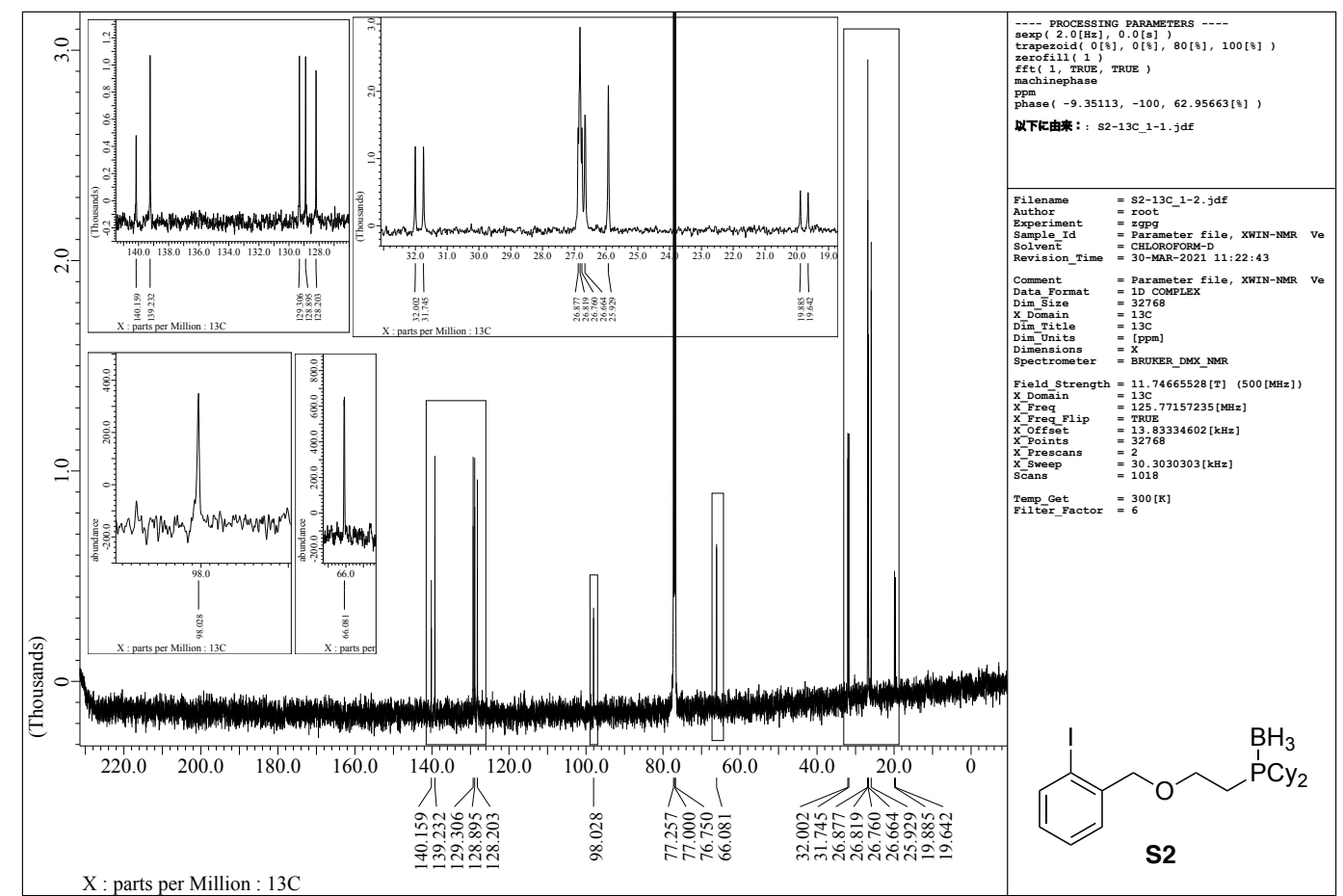

Figure S6. ${ }^{13} \mathrm{C}$ NMR spectrum of $\mathbf{S 2}$ 


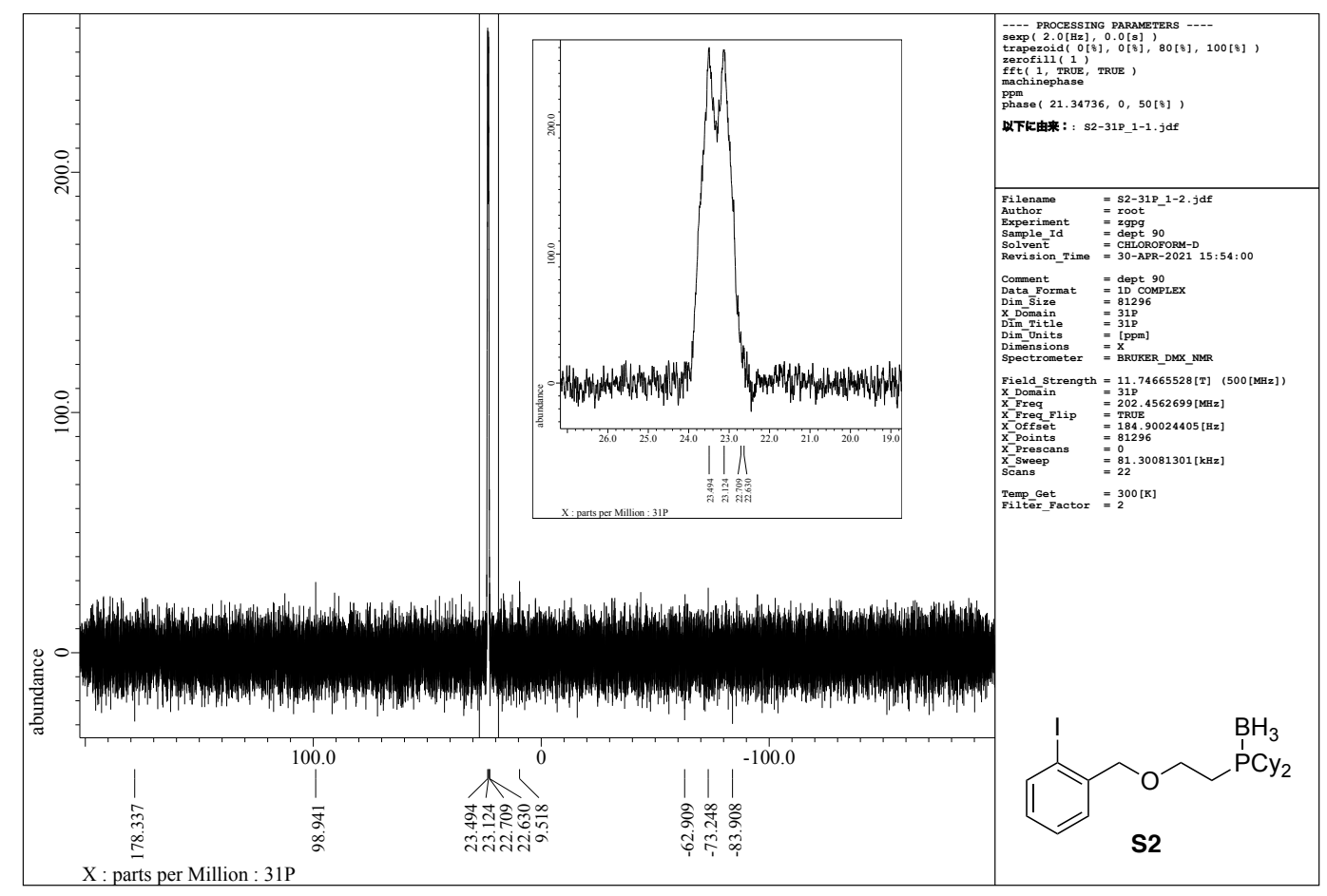

Figure S7. ${ }^{31} \mathrm{P}$ NMR spectrum of $\mathbf{S 2}$

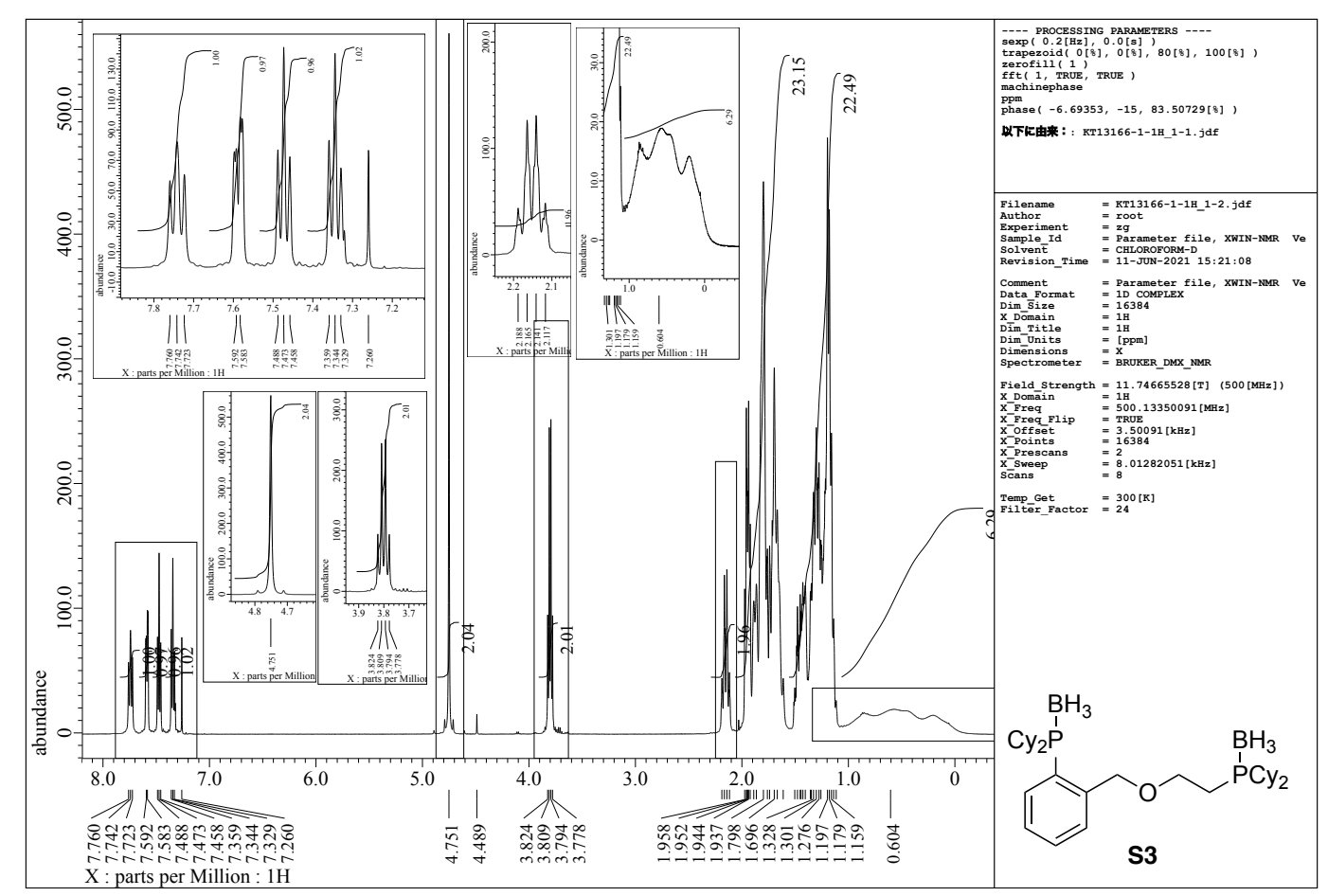

Figure S8. ${ }^{1} \mathrm{H}$ NMR spectrum of $\mathbf{S 3}$ 


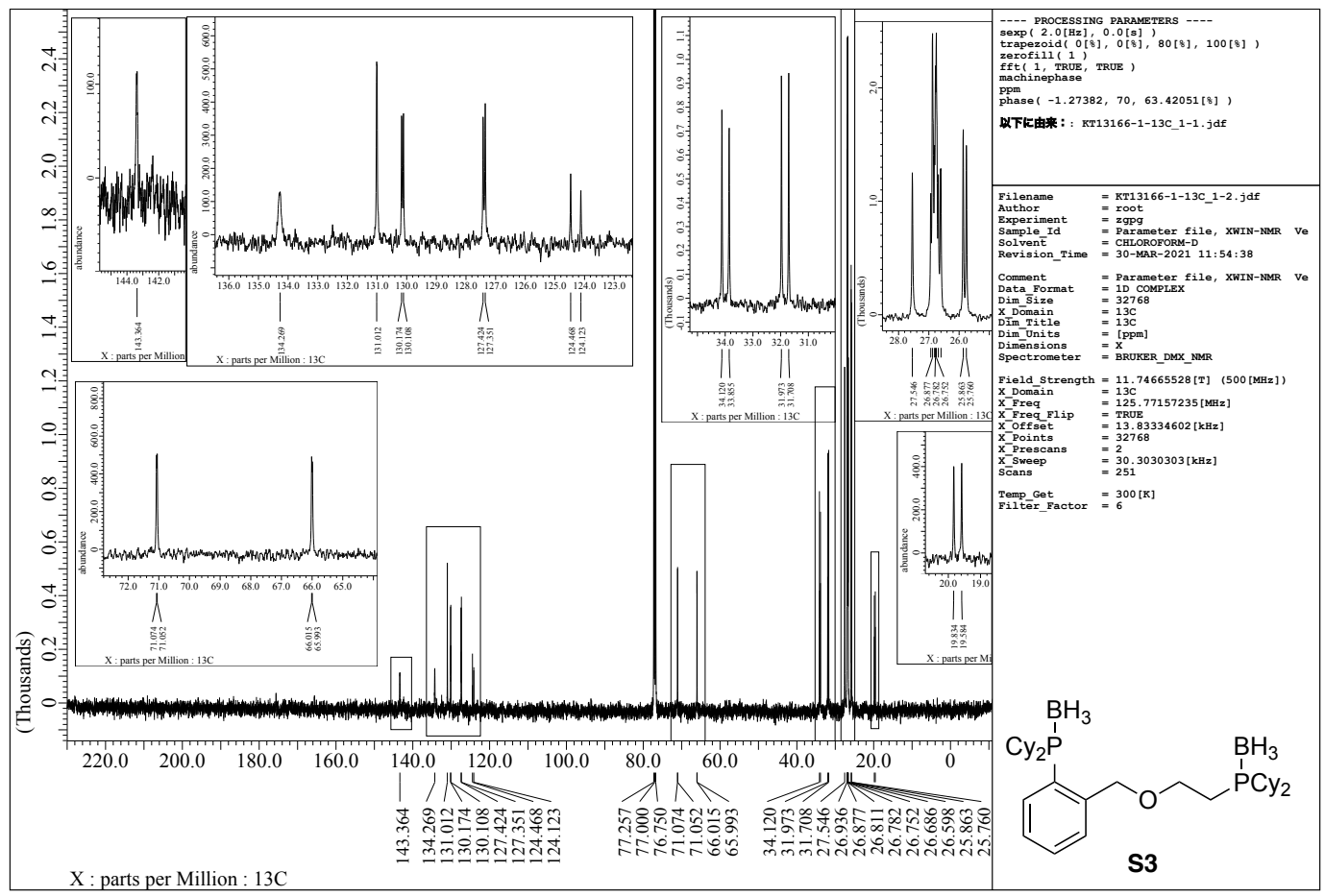

Figure S9. ${ }^{13} \mathrm{C}$ NMR spectrum of $\mathbf{S 3}$

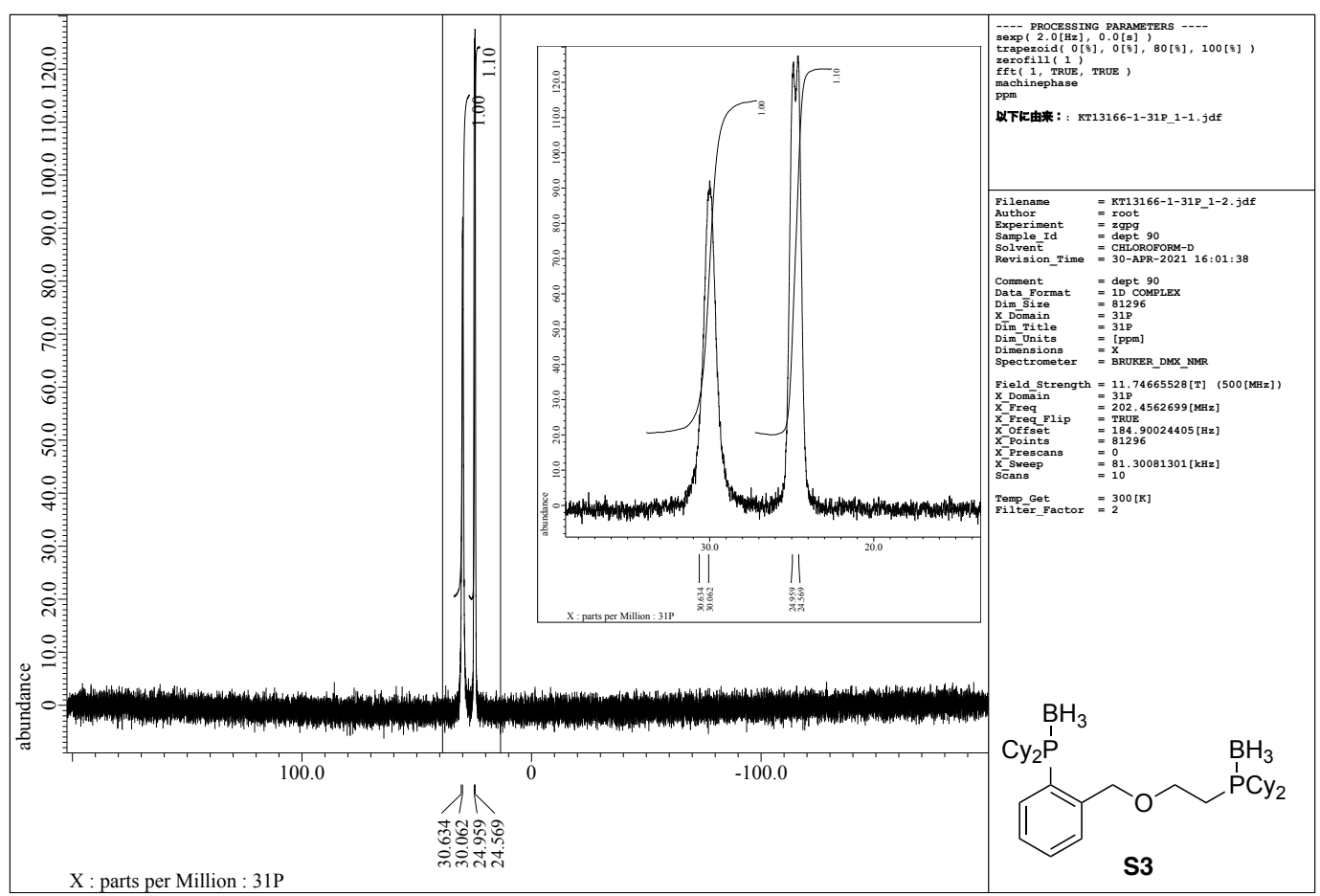

Figure S10. ${ }^{31} \mathrm{P}$ NMR spectrum of $\mathbf{S 3}$ 


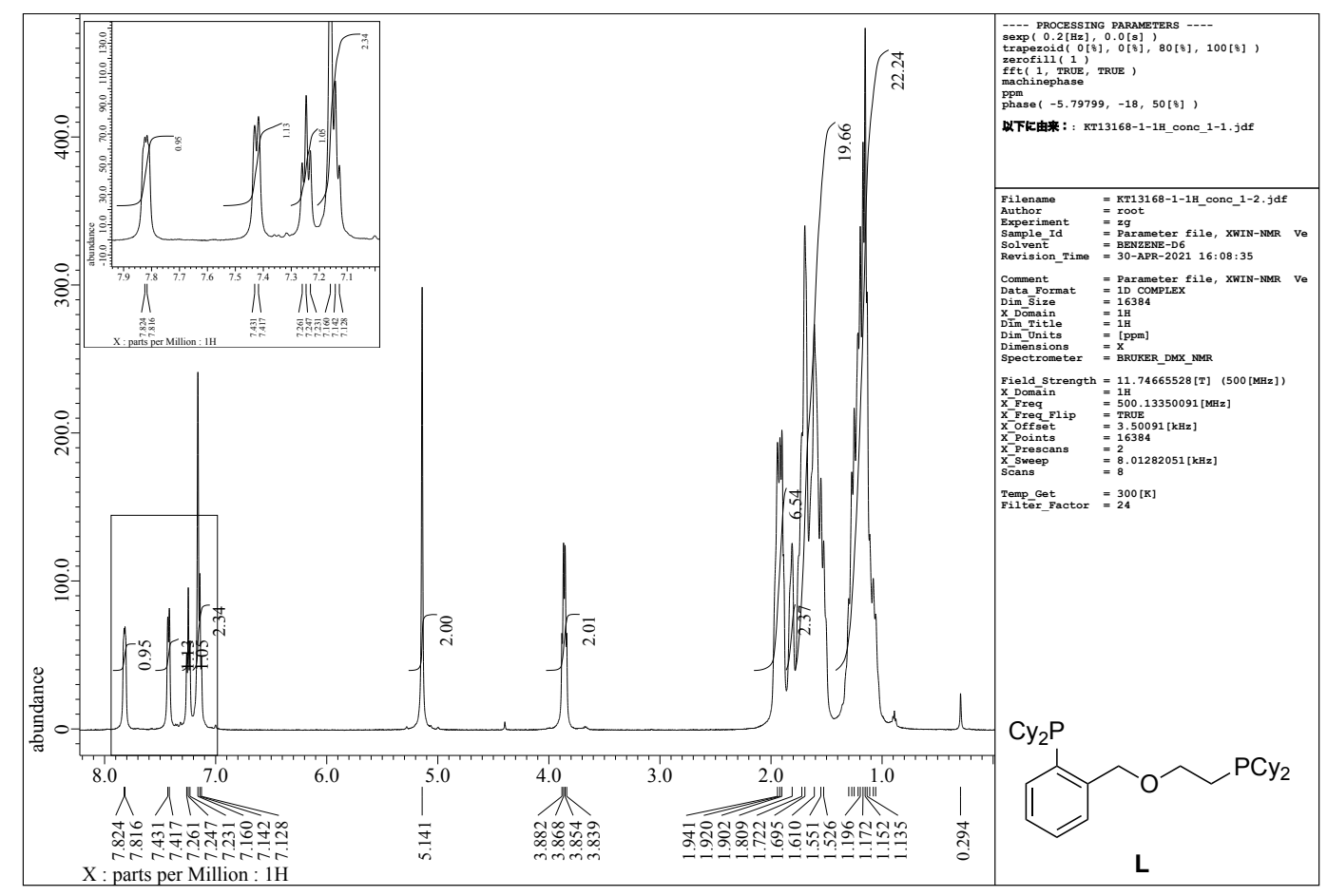

Figure S11. ${ }^{1} \mathrm{H}$ NMR spectrum of $\mathbf{L}$

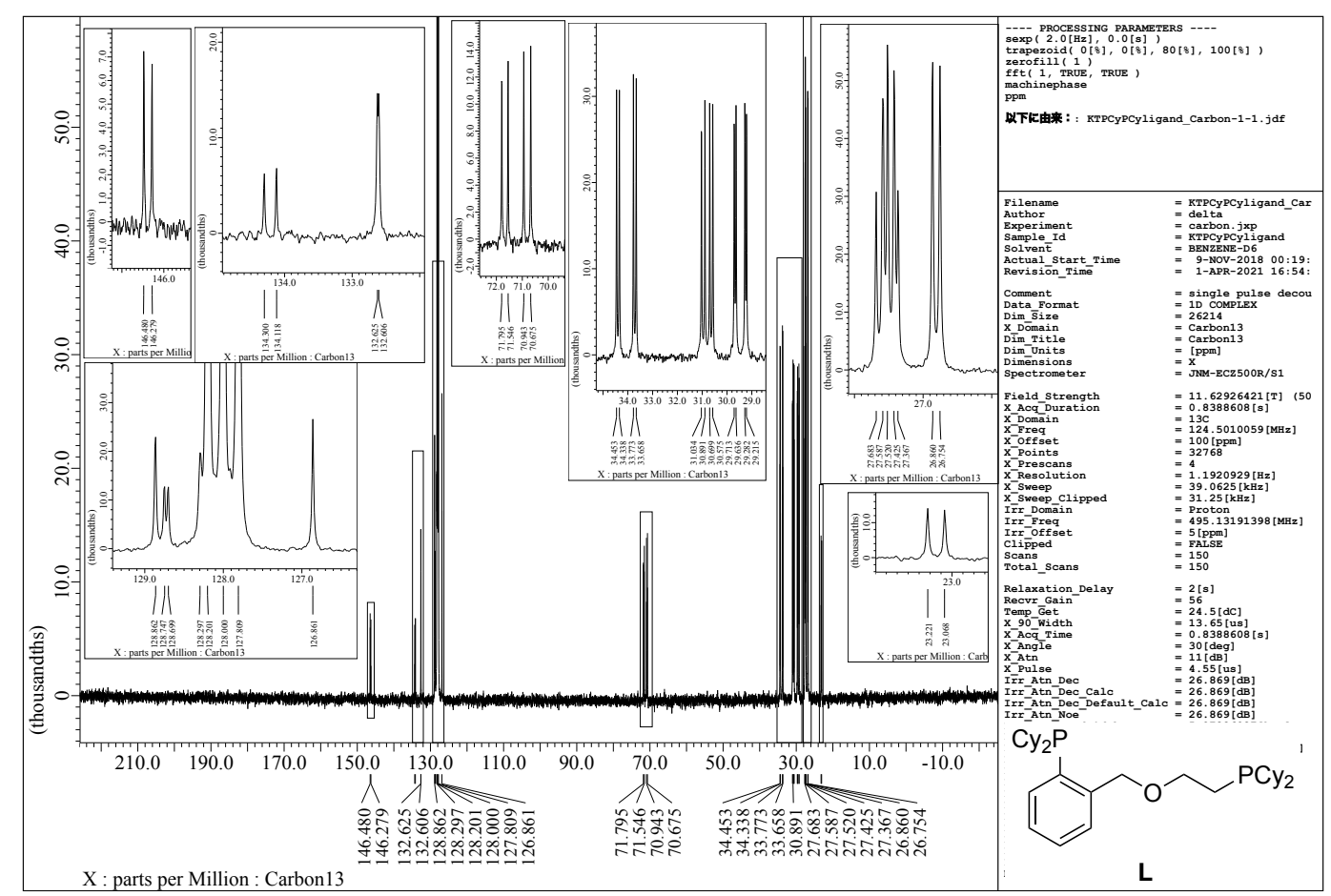

Figure S12. ${ }^{13} \mathrm{C}$ NMR spectrum of $\mathbf{L}$ 


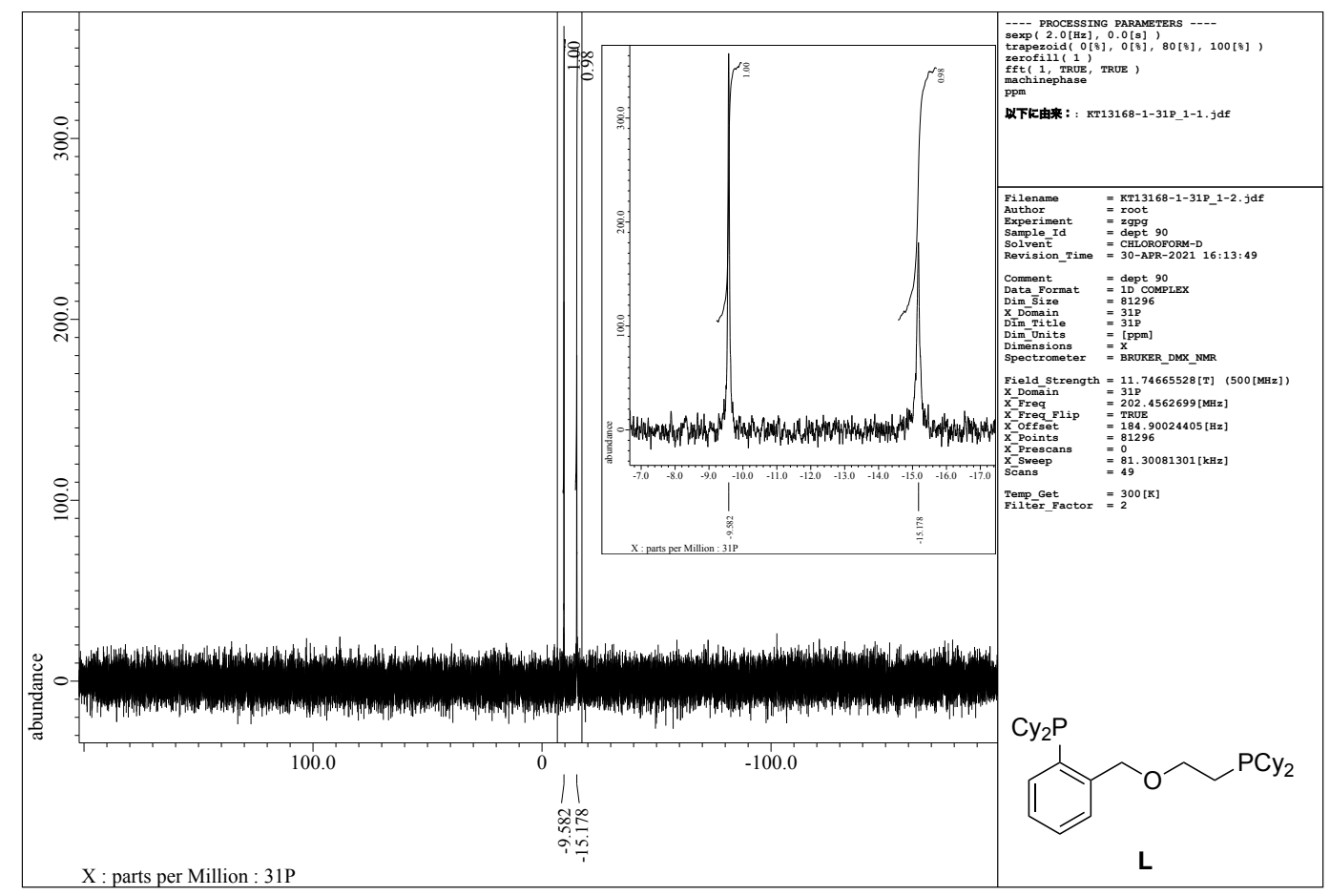

Figure S13. ${ }^{31} \mathrm{P}$ NMR spectrum of $\mathbf{L}$

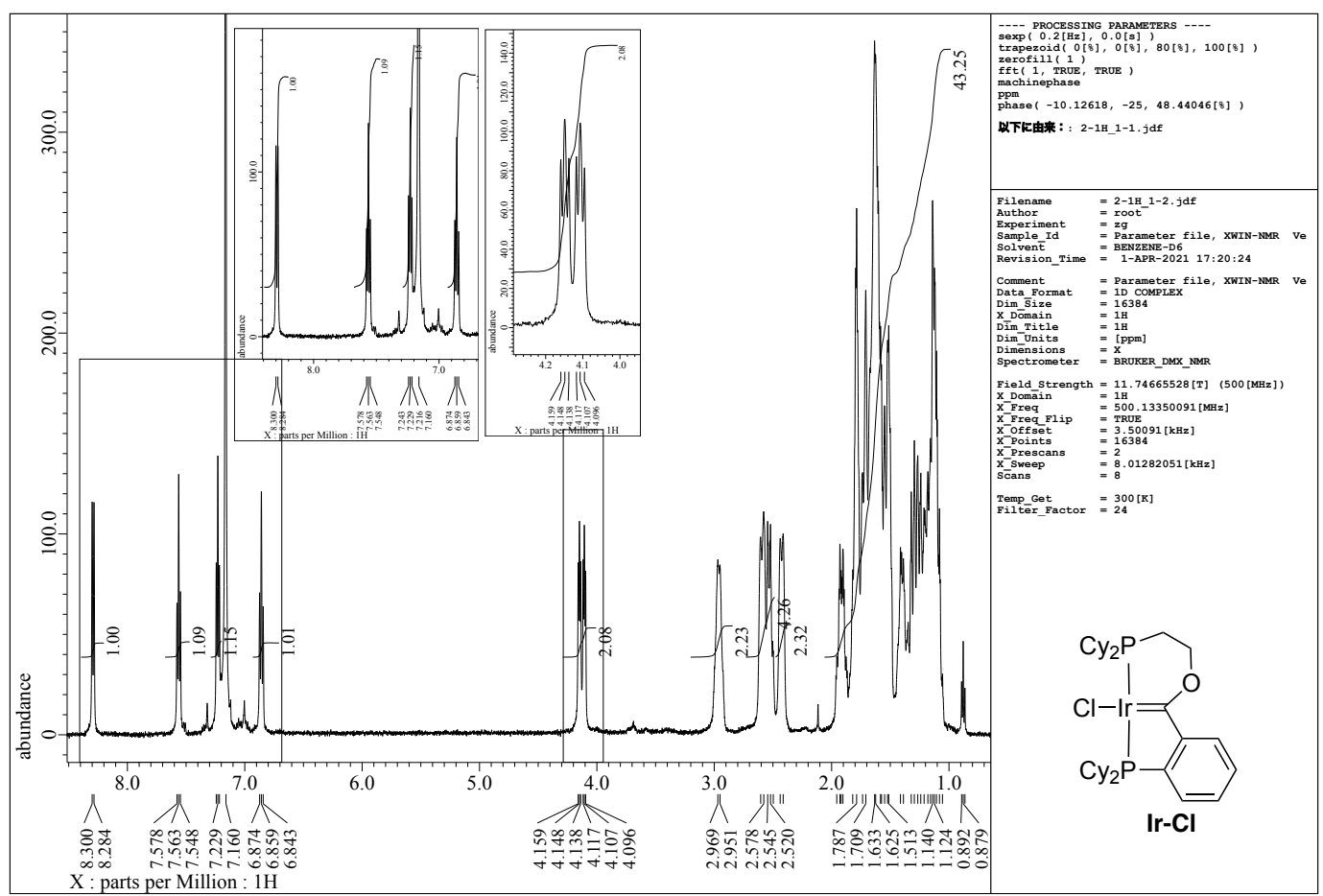

Figure S14. ${ }^{1} \mathrm{H}$ NMR spectrum of Ir-Cl 


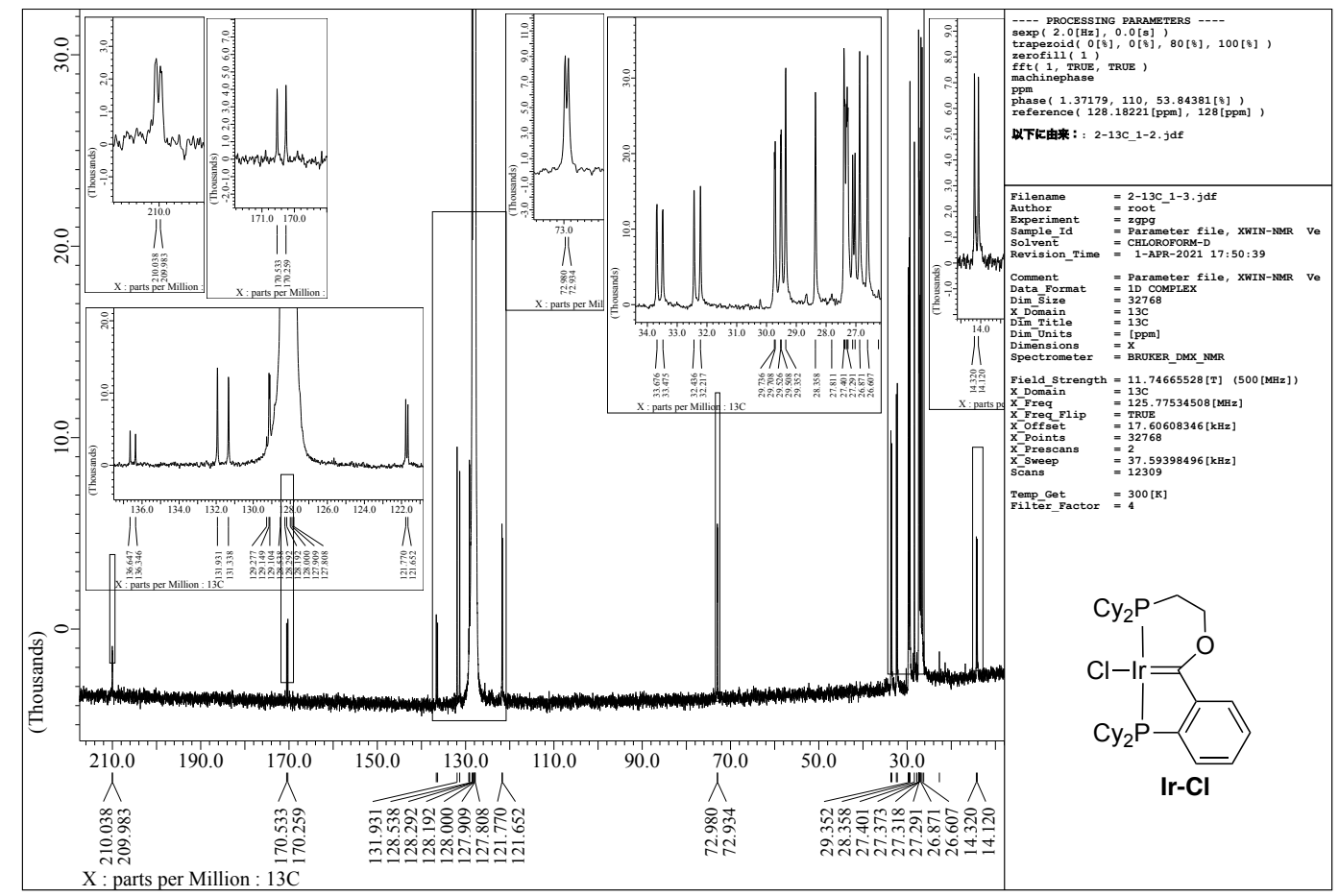

Figure S15. ${ }^{13} \mathrm{C}$ NMR spectrum of Ir-Cl

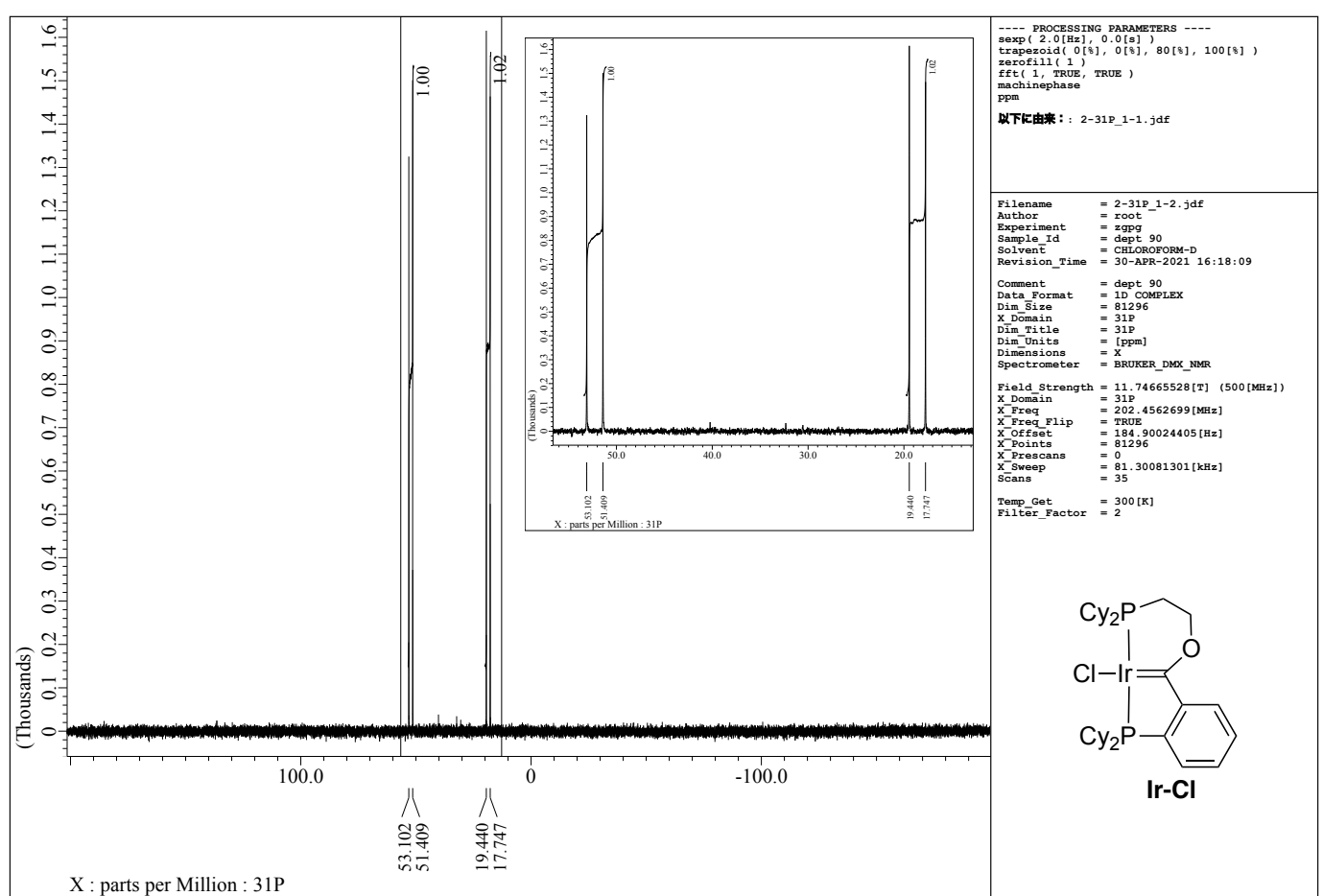

Figure S16. ${ }^{31} \mathrm{P}$ NMR spectrum of $\mathbf{I r}-\mathbf{C l}$ 


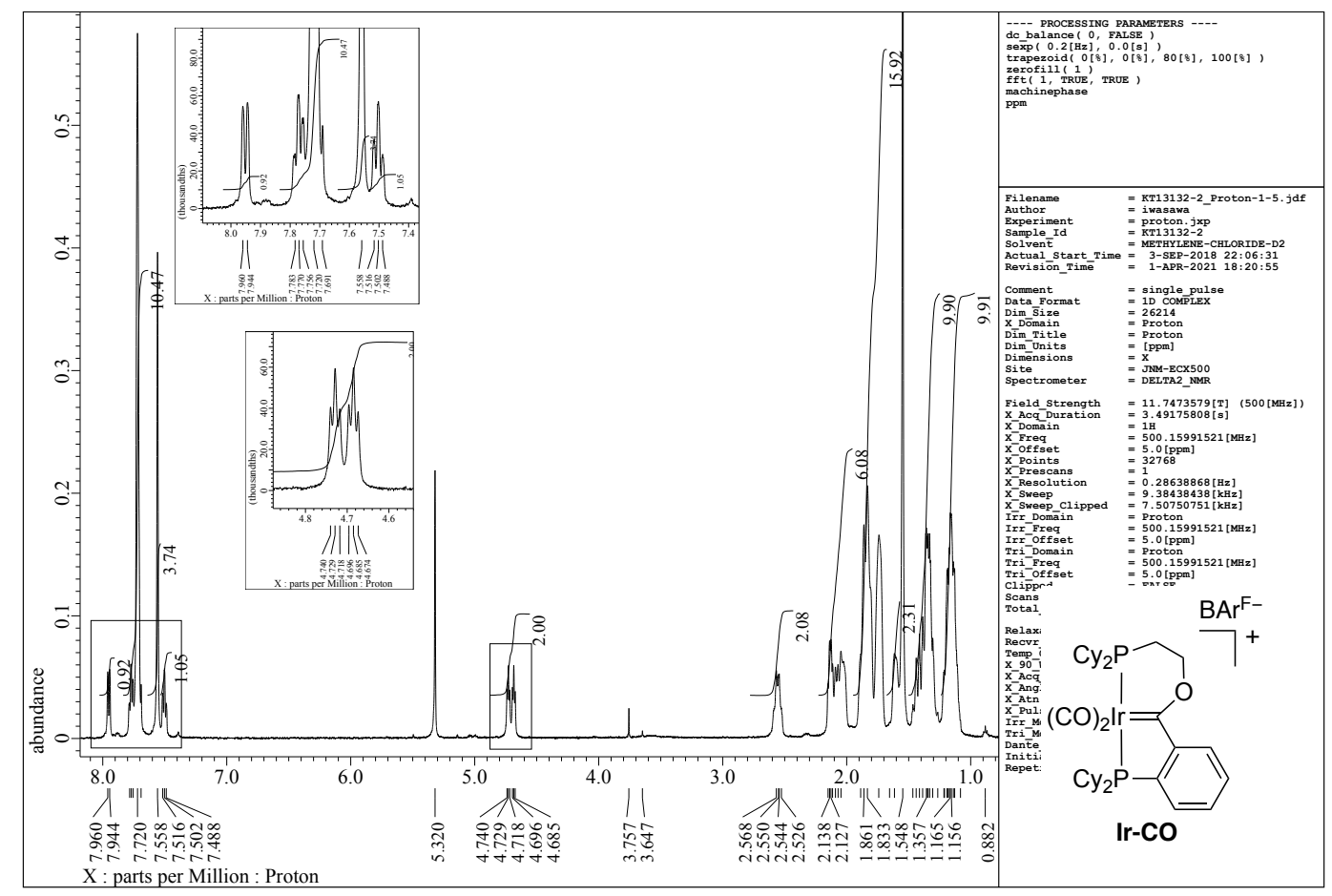

Figure S17. ${ }^{1} \mathrm{H}$ NMR spectrum of Ir-CO

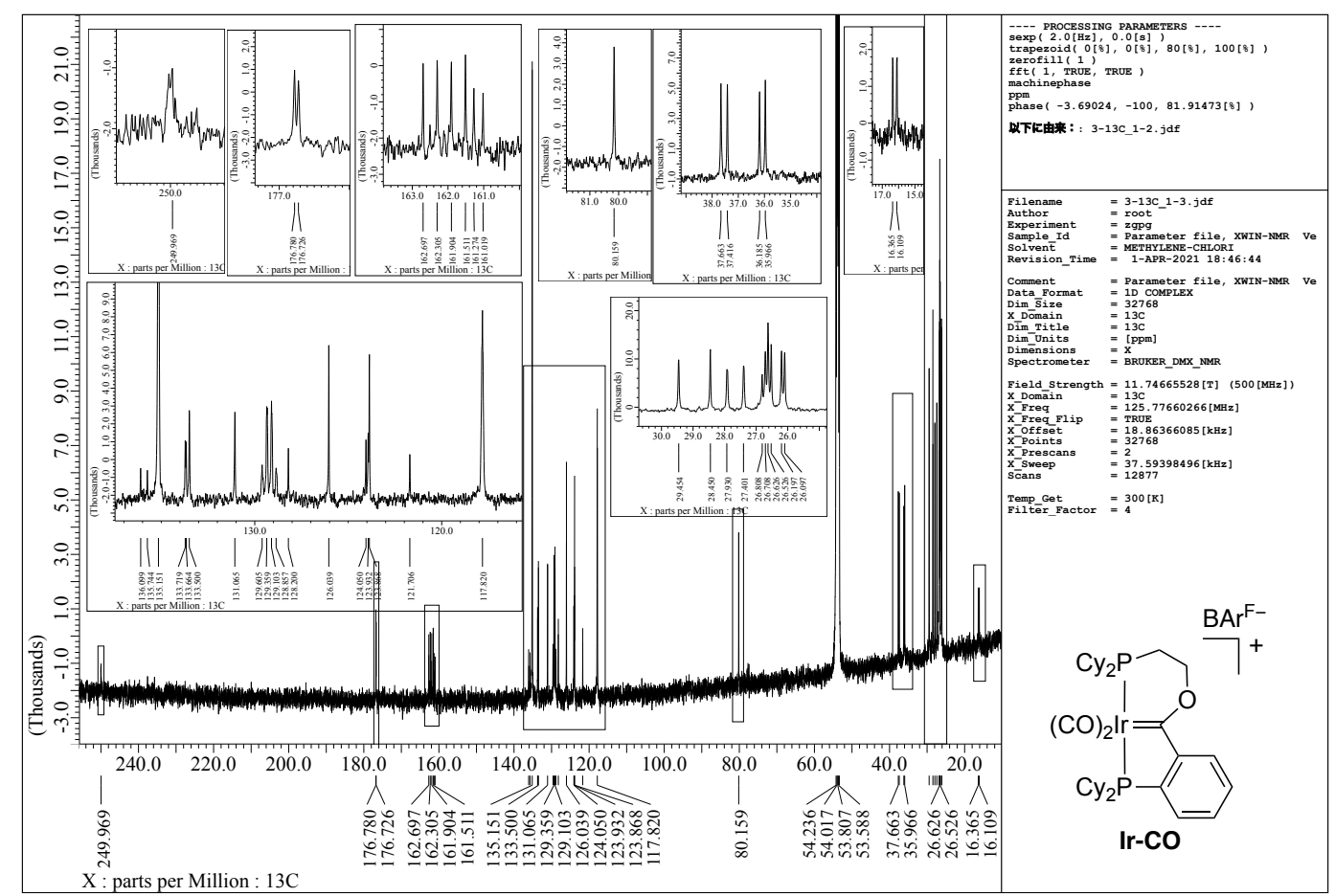

Figure S18. ${ }^{13} \mathrm{C}$ NMR spectrum of Ir-CO 


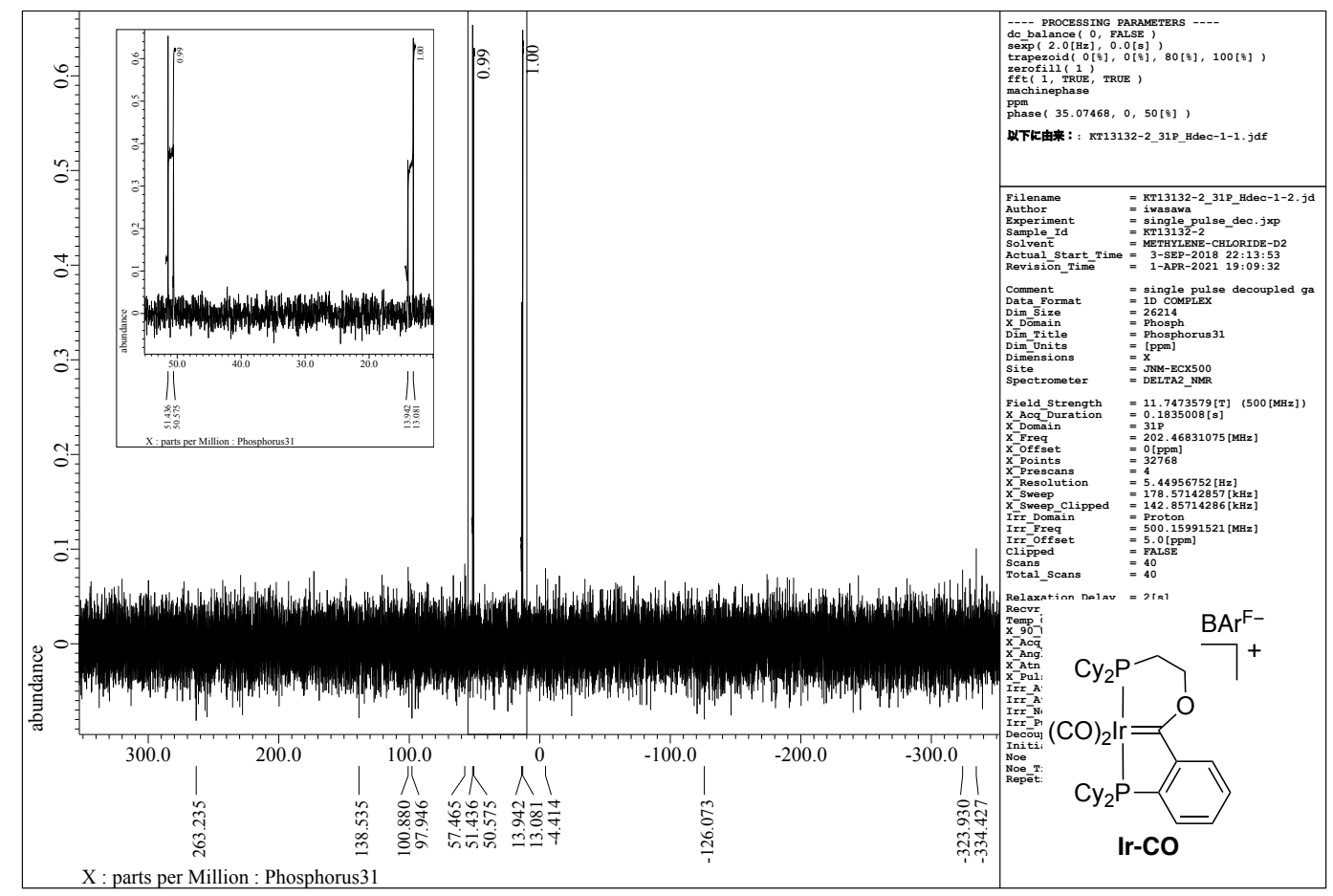

Figure S19. ${ }^{31} \mathrm{P}$ NMR spectrum of Ir-CO

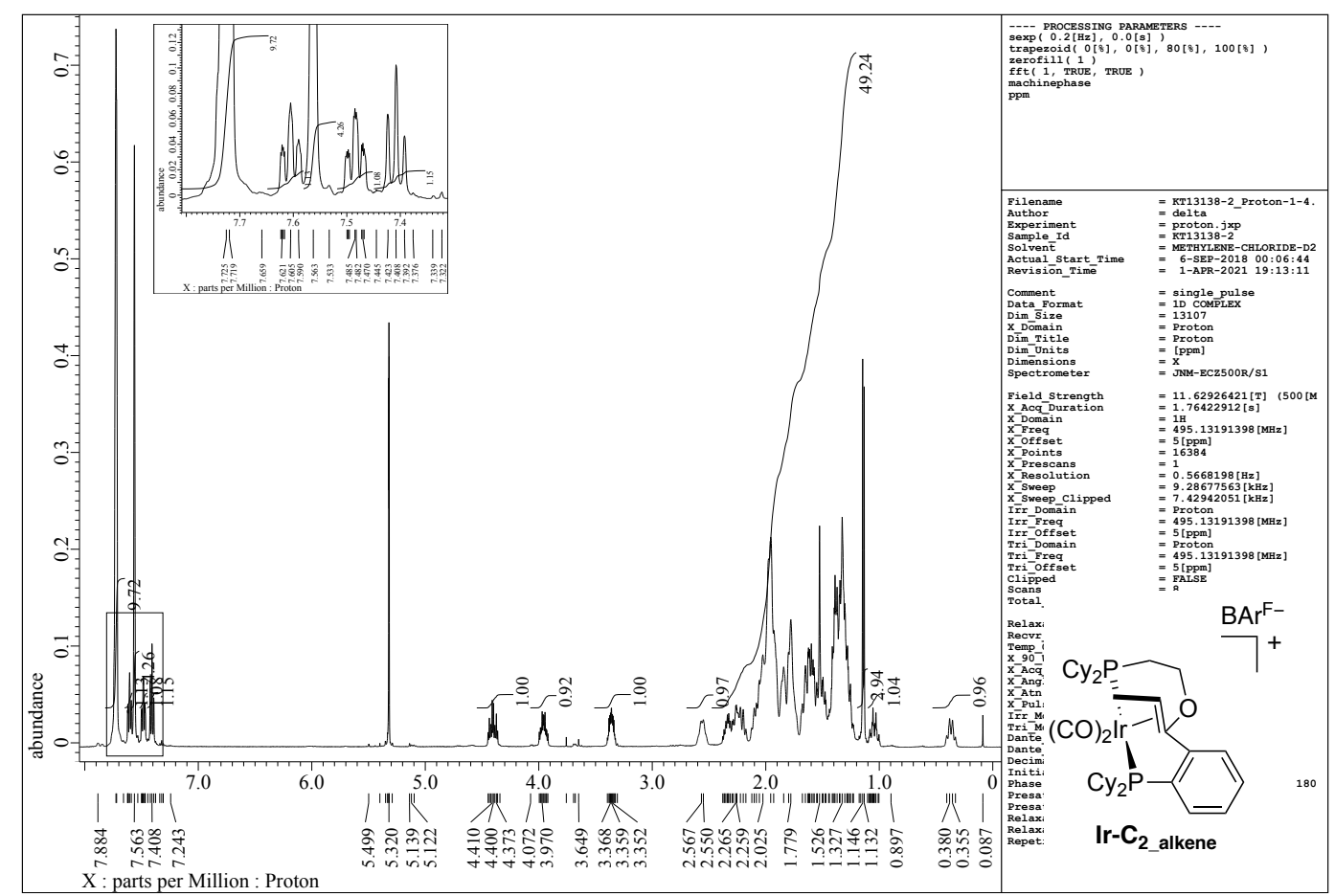

Figure S20. ${ }^{1} \mathrm{H}$ NMR spectrum of $\mathbf{I r}-\mathbf{C}_{2}$ alkene $\left(\mathrm{CD}_{2} \mathrm{Cl}_{2}\right)$ 


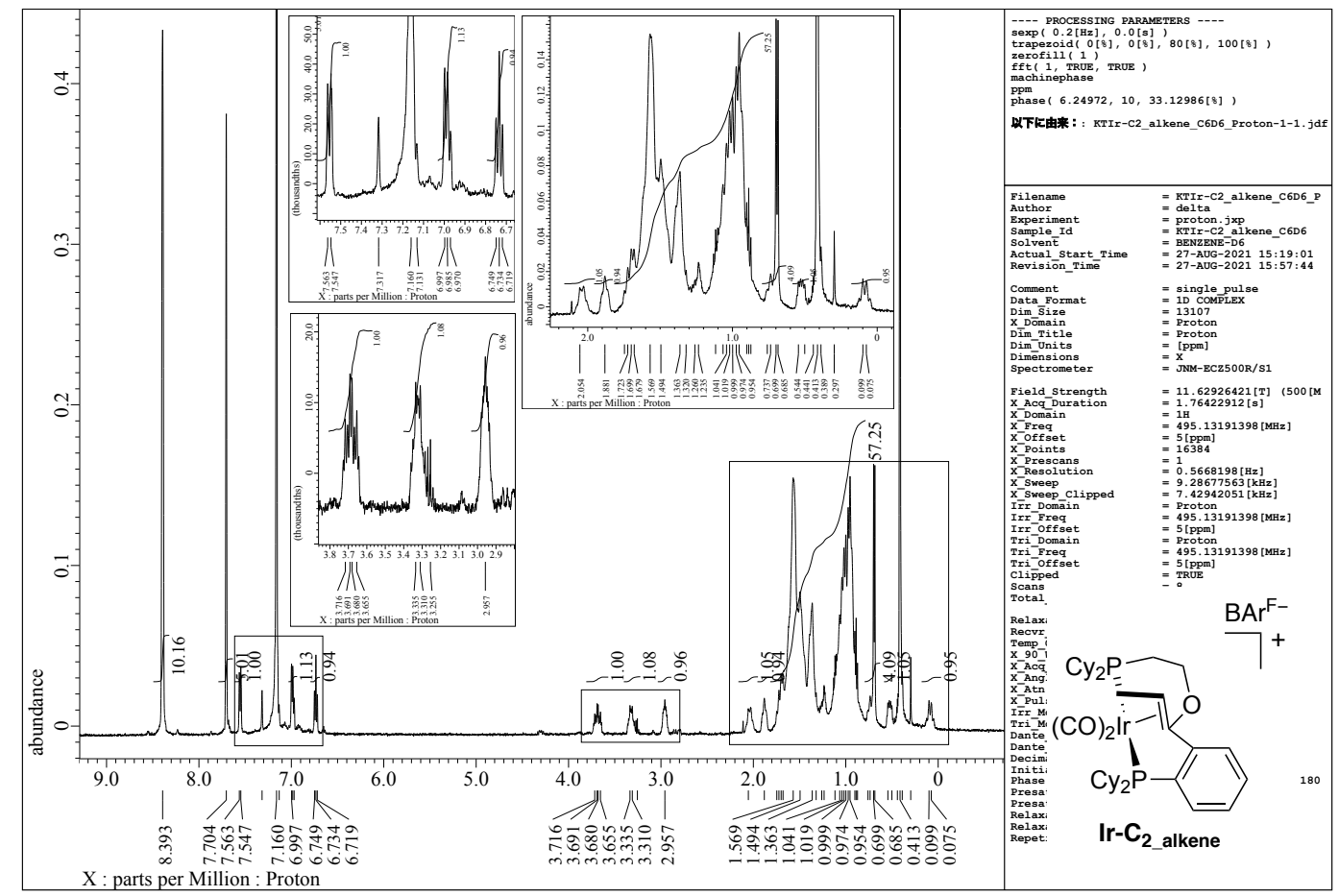

Figure S21. ${ }^{1} \mathrm{H}$ NMR spectrum of Ir- $\mathbf{C}_{2}$ alkene $\left(\mathrm{C}_{6} \mathrm{D}_{6}\right)$

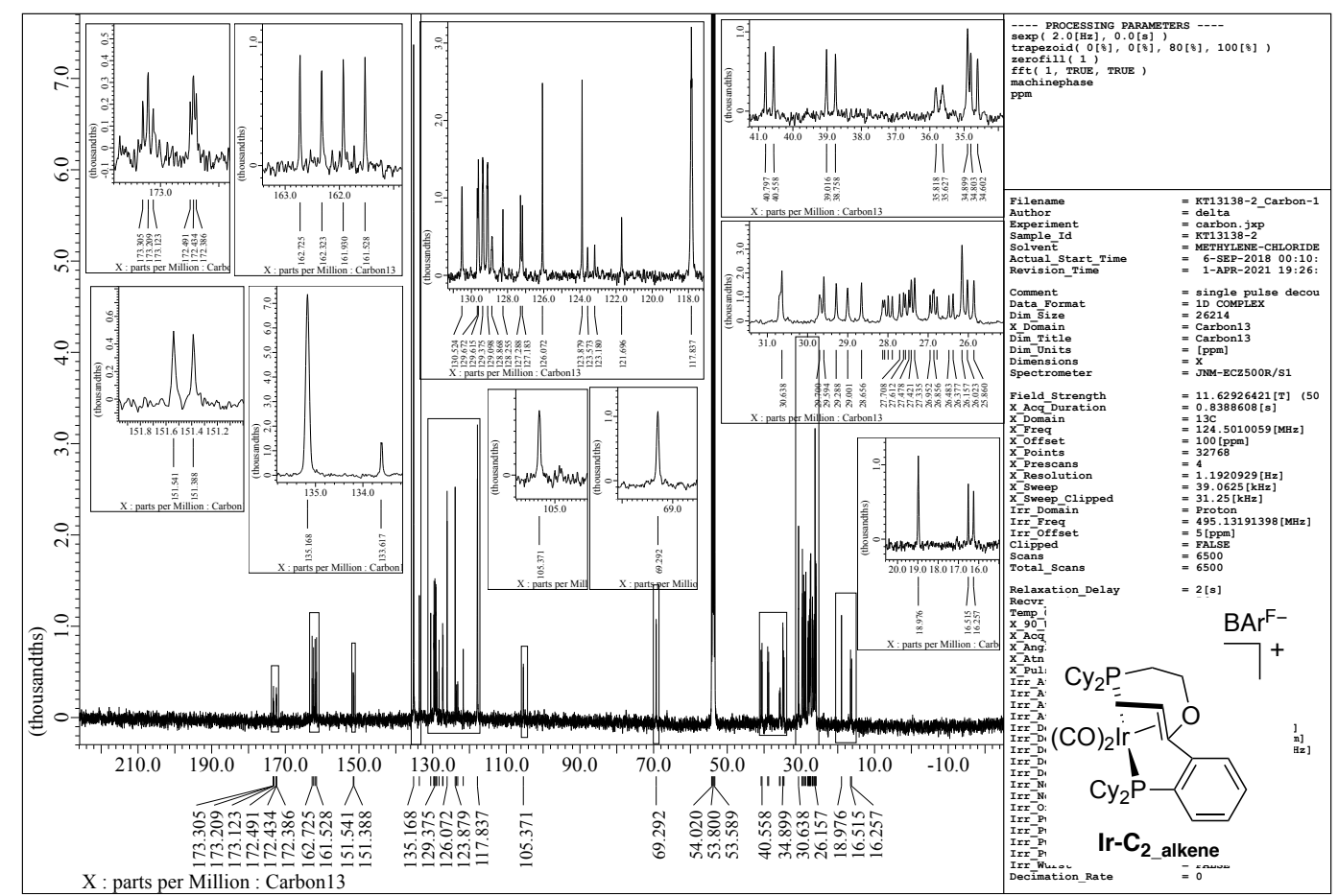

Figure S22. ${ }^{13} \mathrm{C}$ NMR spectrum of Ir- $\mathbf{C}_{2}$ alkene $\left(\mathrm{CD}_{2} \mathrm{Cl}_{2}\right)$ 


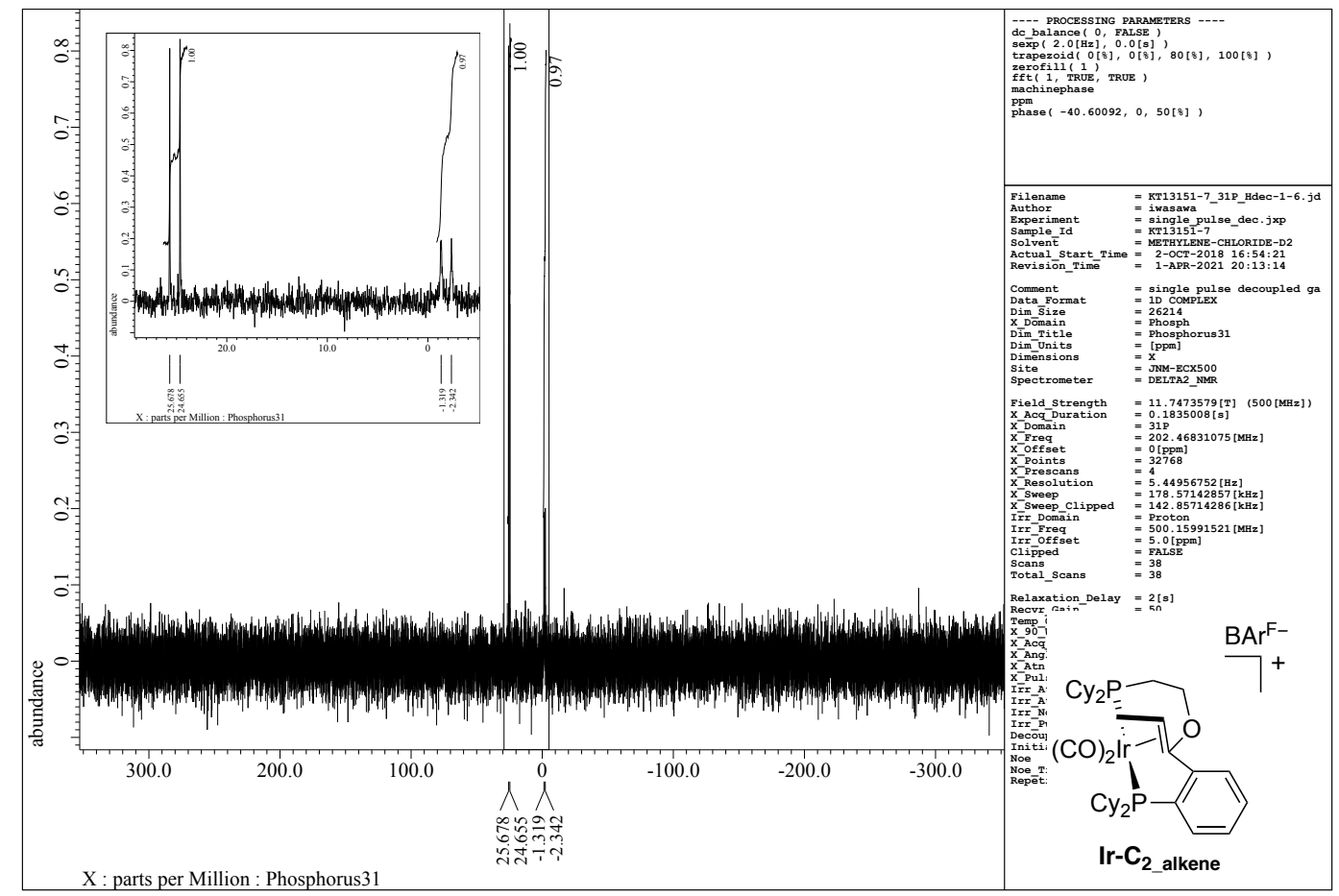

Figure S23. ${ }^{31} \mathrm{P}$ NMR spectrum of Ir-C $\mathbf{C}_{2}$ alkene $\left(\mathrm{CD}_{2} \mathrm{Cl}_{2}\right)$

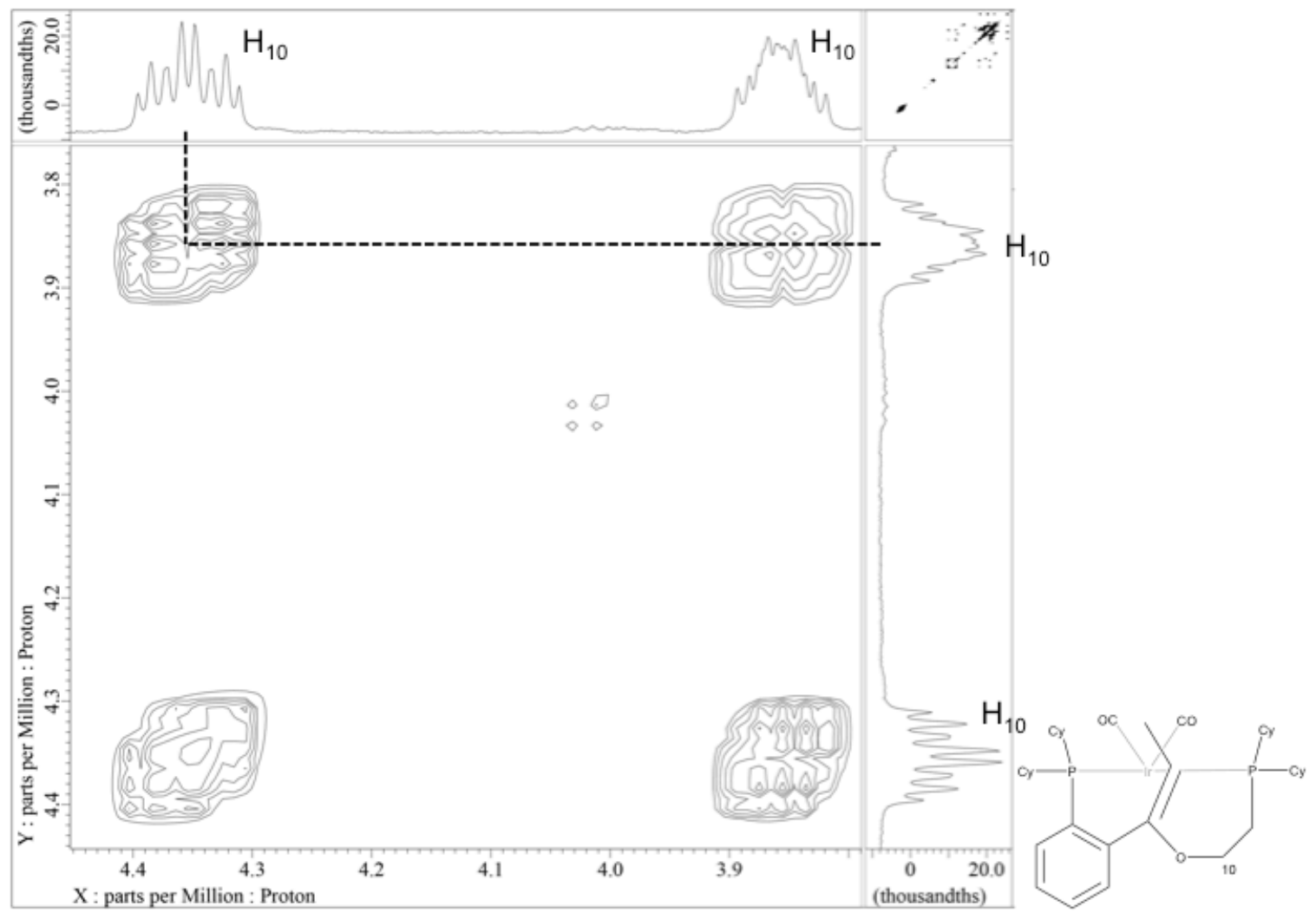

Figure S24. Selected region-1 of HHCOSY spectrum of $\mathbf{I r - \mathbf { C } _ { 2 } \text { alkene }}$ 

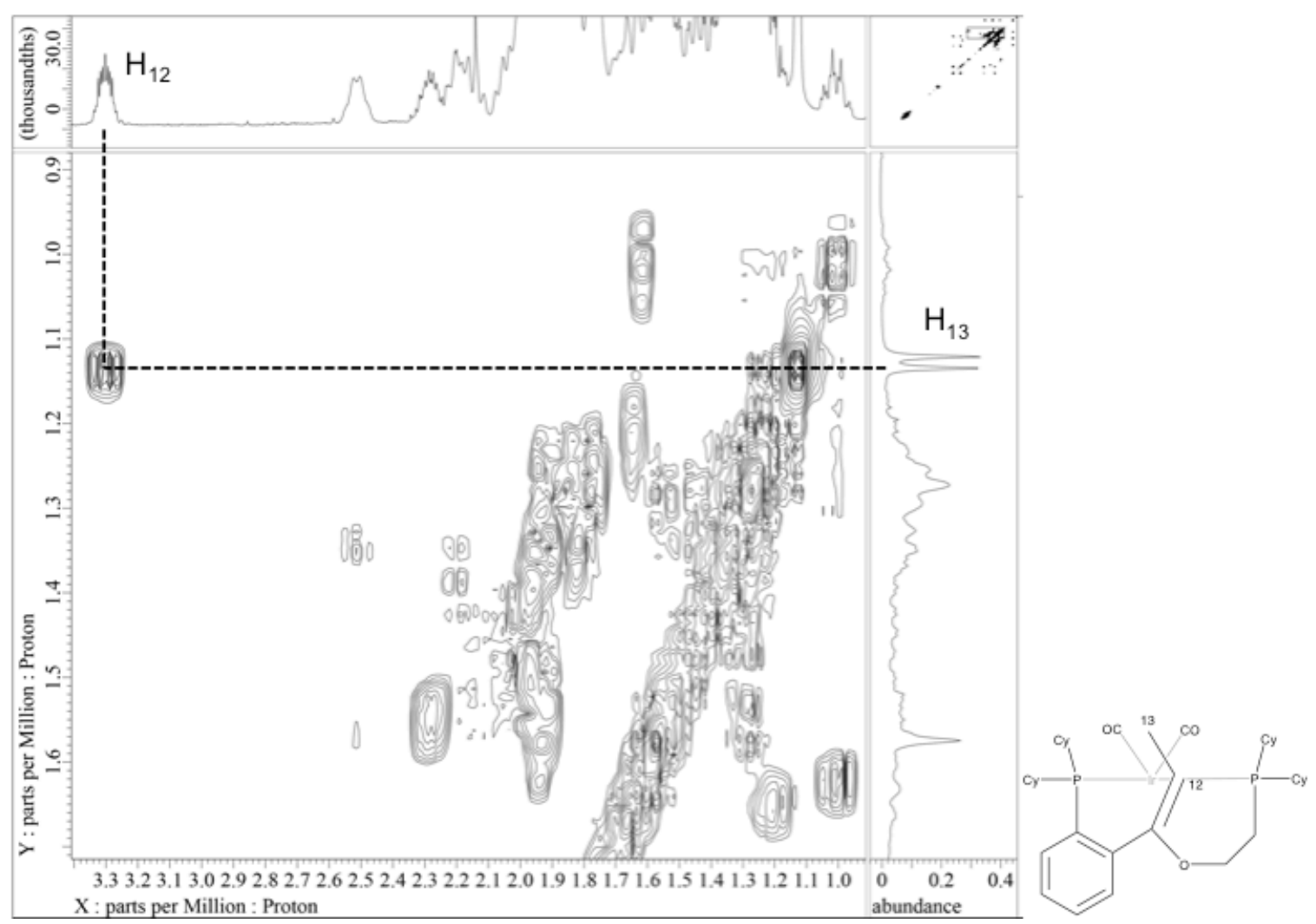

Figure S25. Selected region-2 of HHCOSY spectrum of $\mathbf{I r - \mathbf { C } _ { 2 } \text { alkene }}$
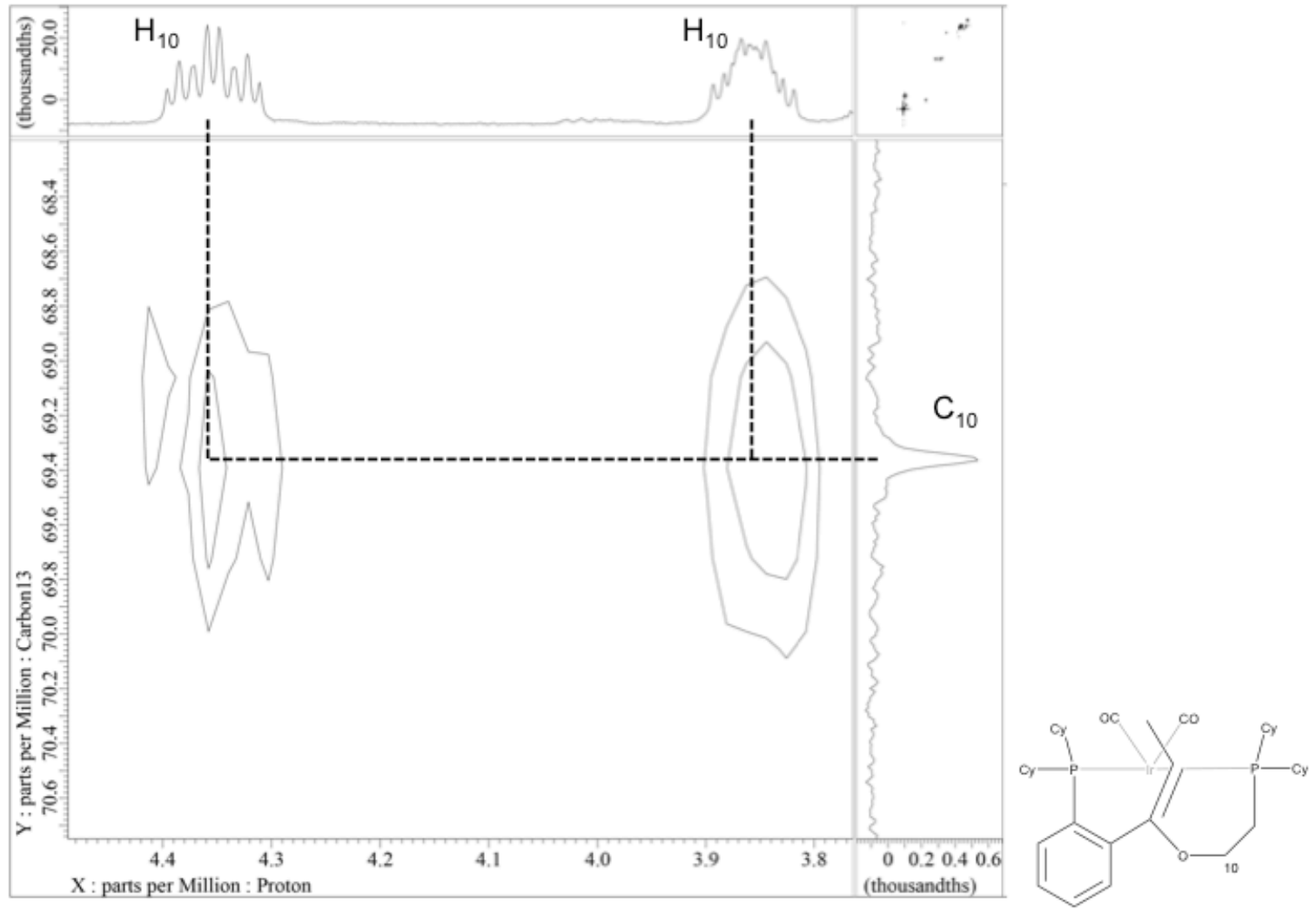

Figure S26. Selected region-1 of HMQC spectrum of Ir-C $\mathbf{2}_{\text {2 alkene }}$ 


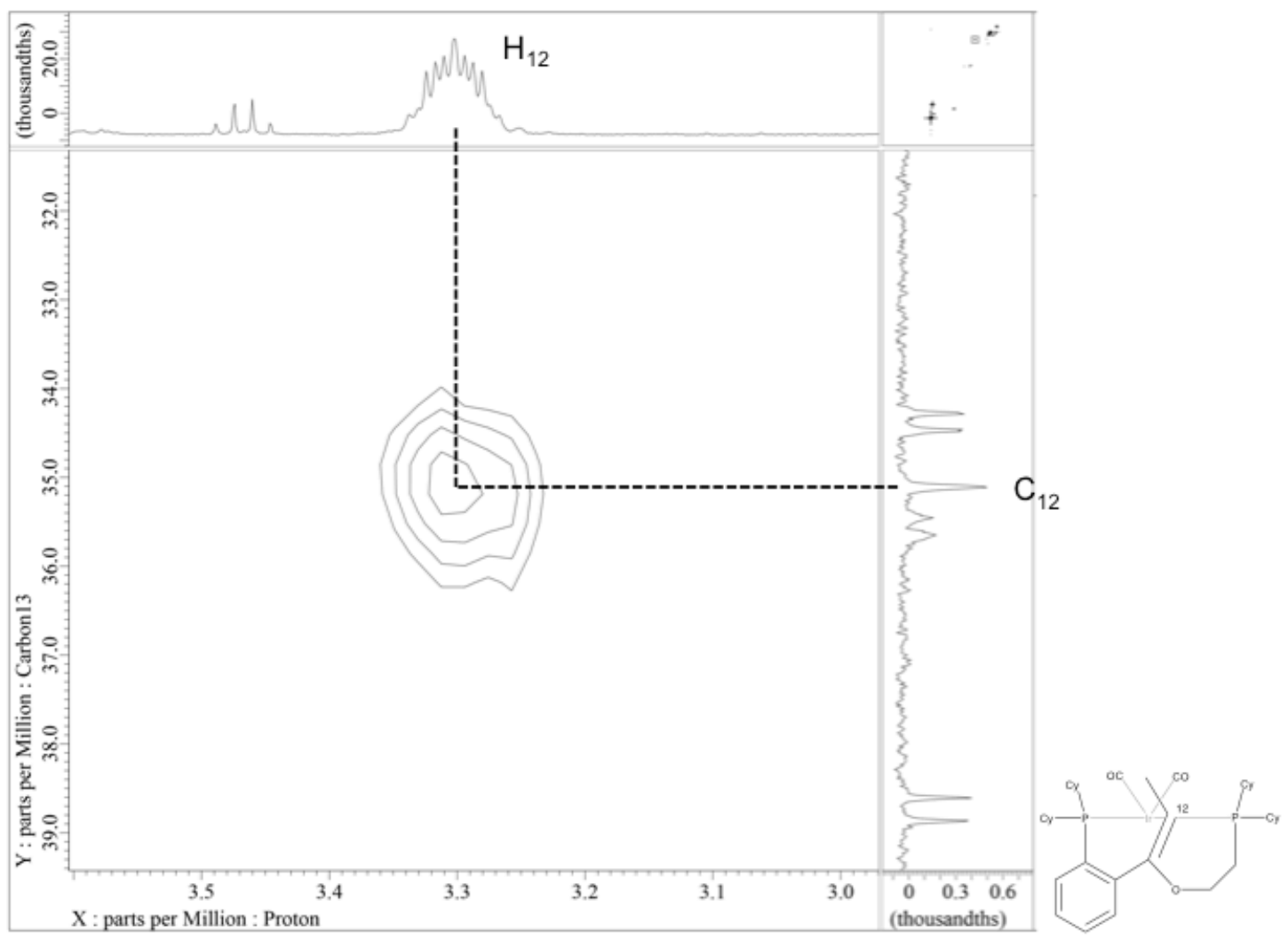

Figure S27. Selected region-2 of HMQC spectrum of Ir-C $\mathbf{C}_{2}$ alkene

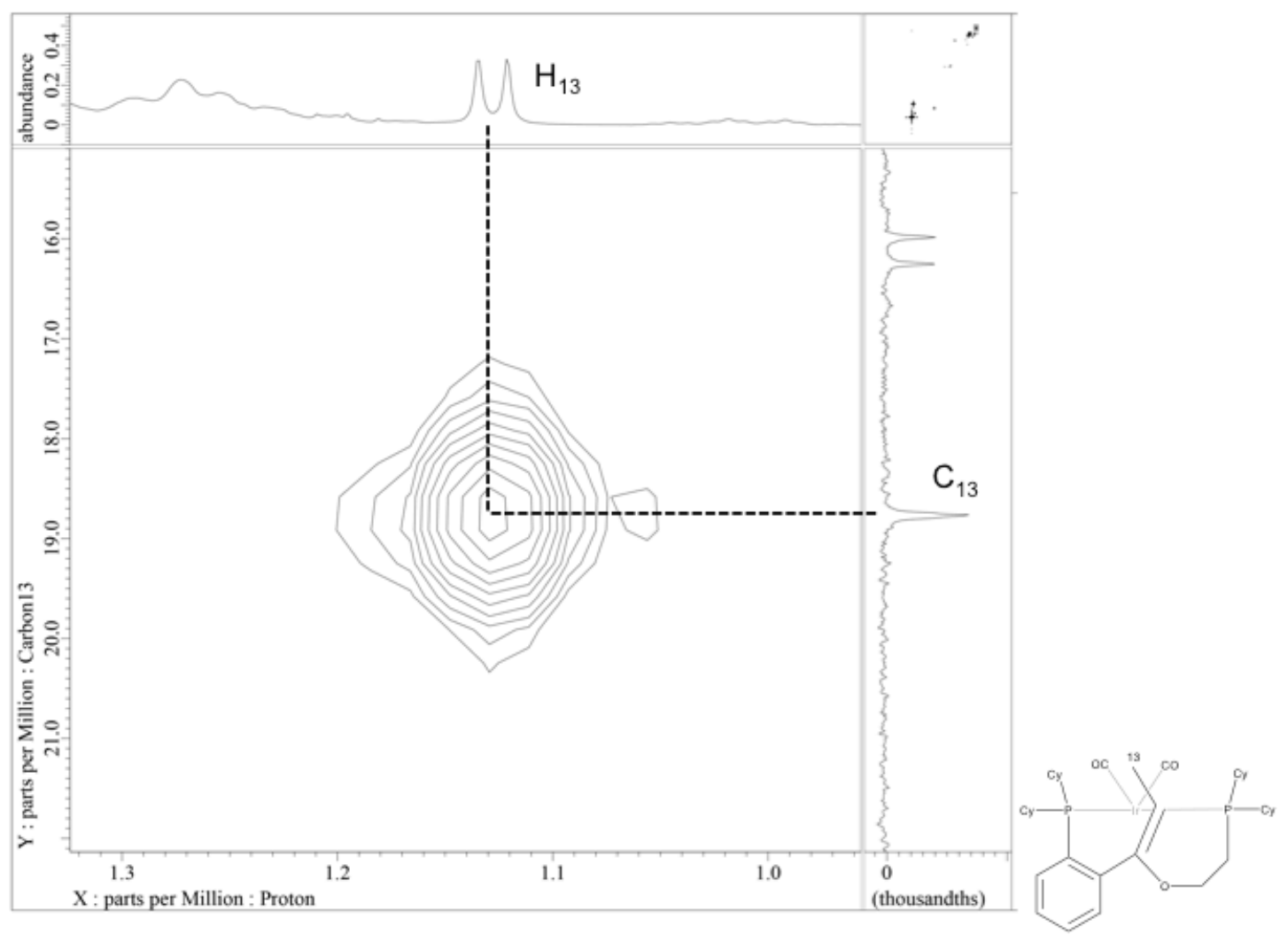

Figure S28. Selected region-3 of HMQC spectrum of Ir- $\mathbf{C}_{\mathbf{2}_{-} \text {alkene }}$ 

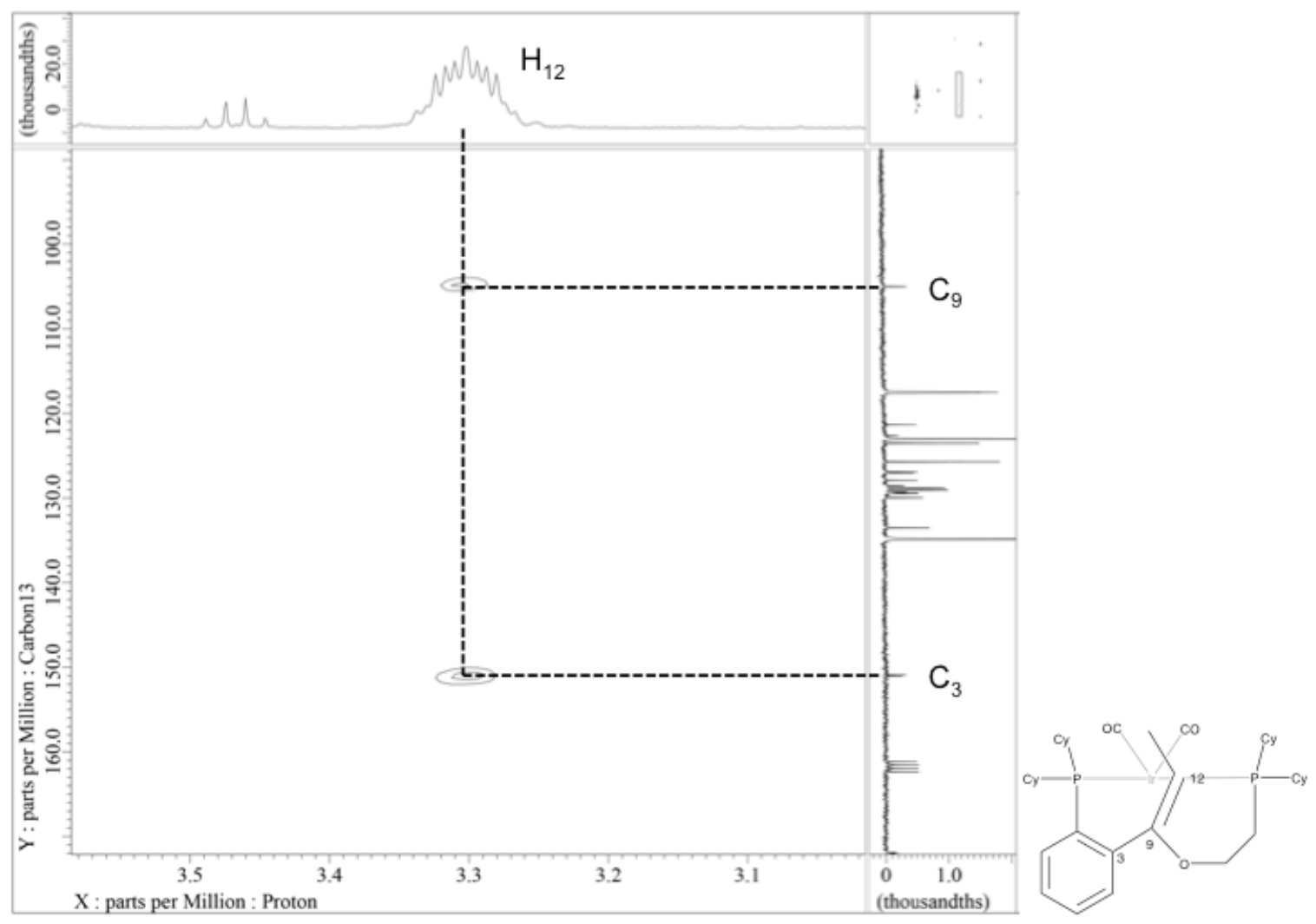

Figure S29. Selected region-1 of HMBC spectrum of Ir-C $\mathbf{2}_{2}$ alkene

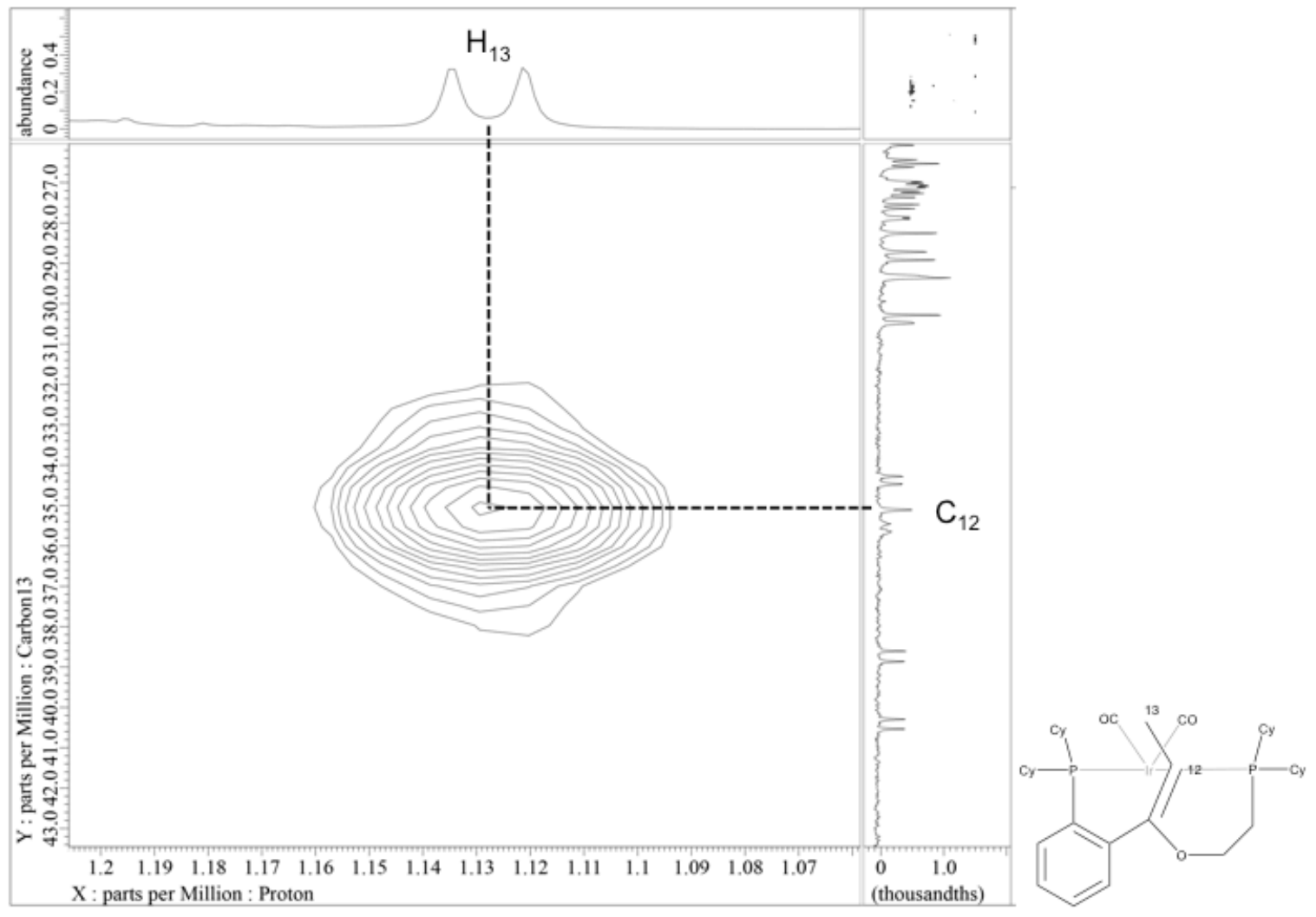

Figure S30. Selected region-2 of HMBC spectrum of Ir- $\mathbf{C}_{\mathbf{2}_{-} \text {alkene }}$ 


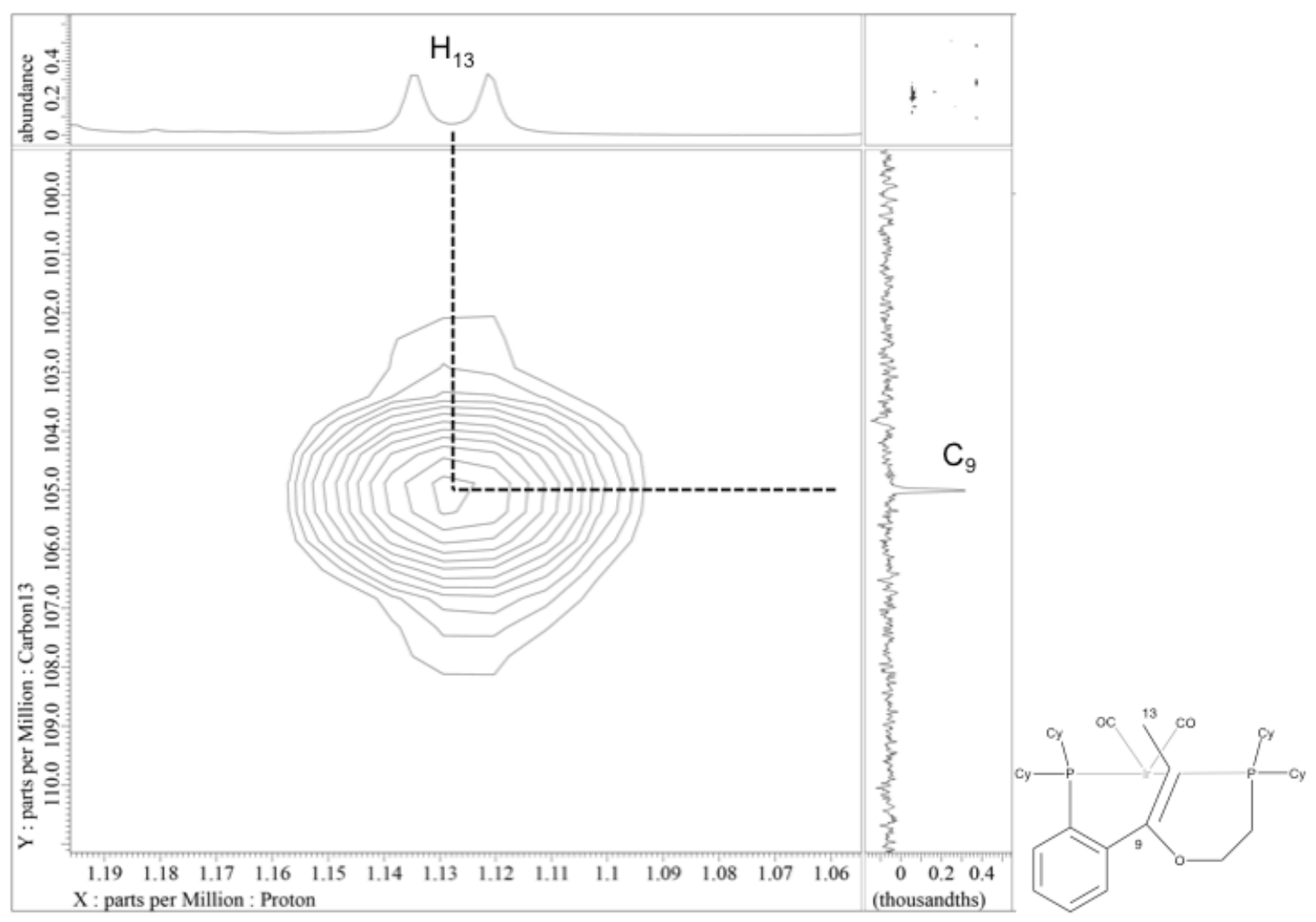

Figure S31. Selected region-3 of HMBC spectrum of Ir-C $\mathbf{2}_{2}$ alkene

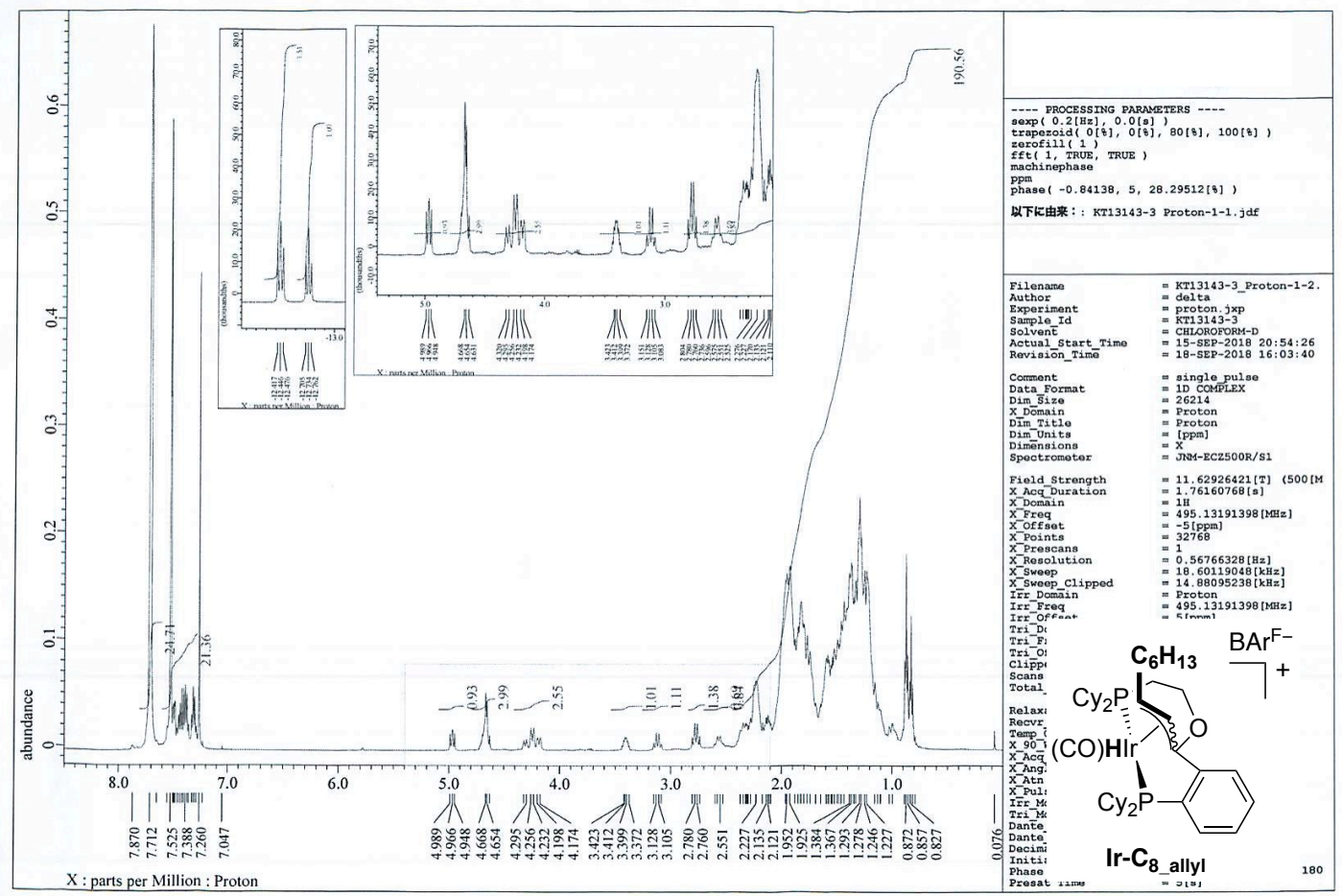

Figure S32. ${ }^{1} \mathrm{H}$ NMR spectrum of $\mathbf{I r}-\mathbf{C}_{\boldsymbol{8}_{-} \text {allyl }}$ 


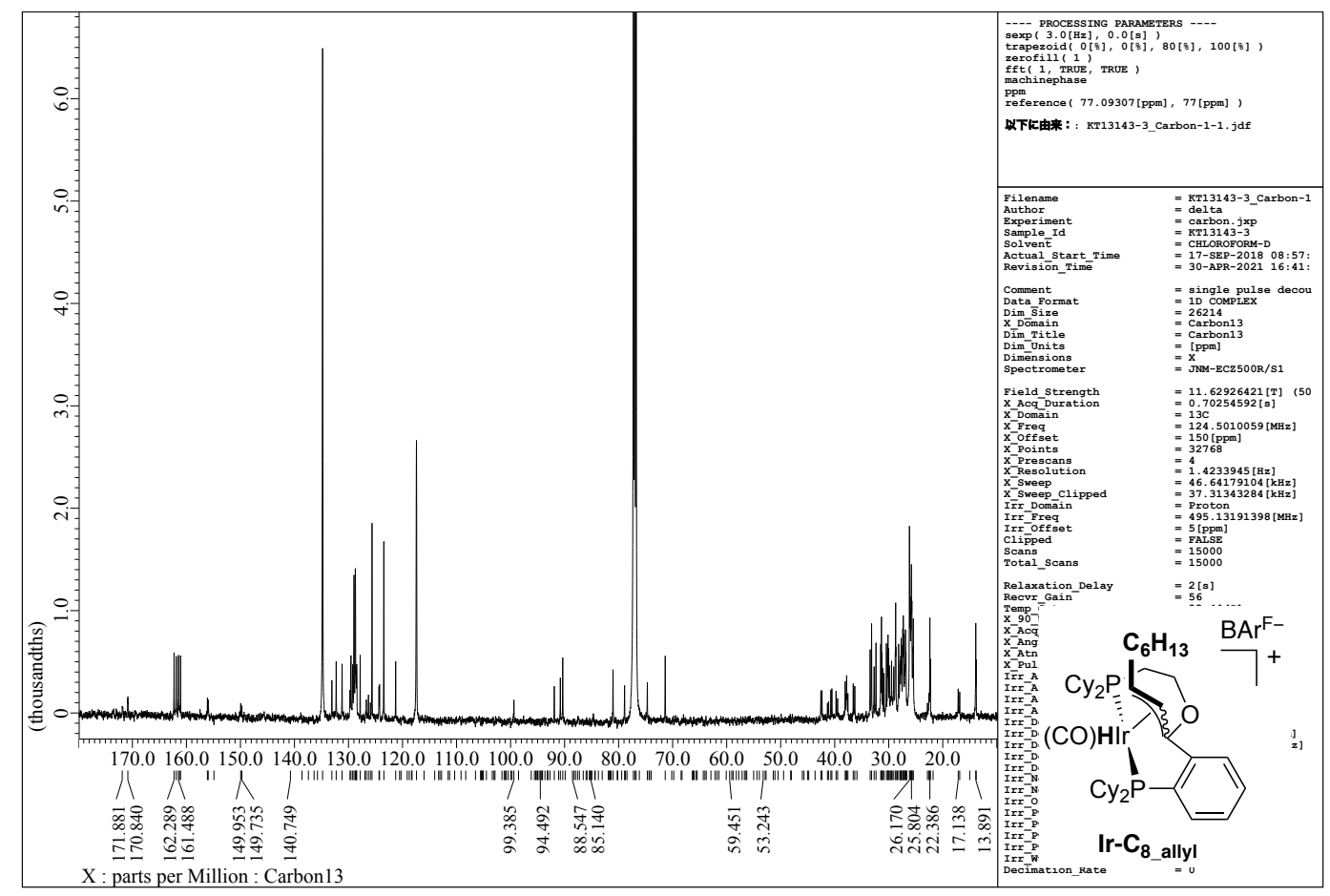

Figure S33. ${ }^{13} \mathrm{C}$ NMR spectrum of $\mathbf{I r}-\mathbf{C}_{\boldsymbol{8}_{-} \text {allyl }}$

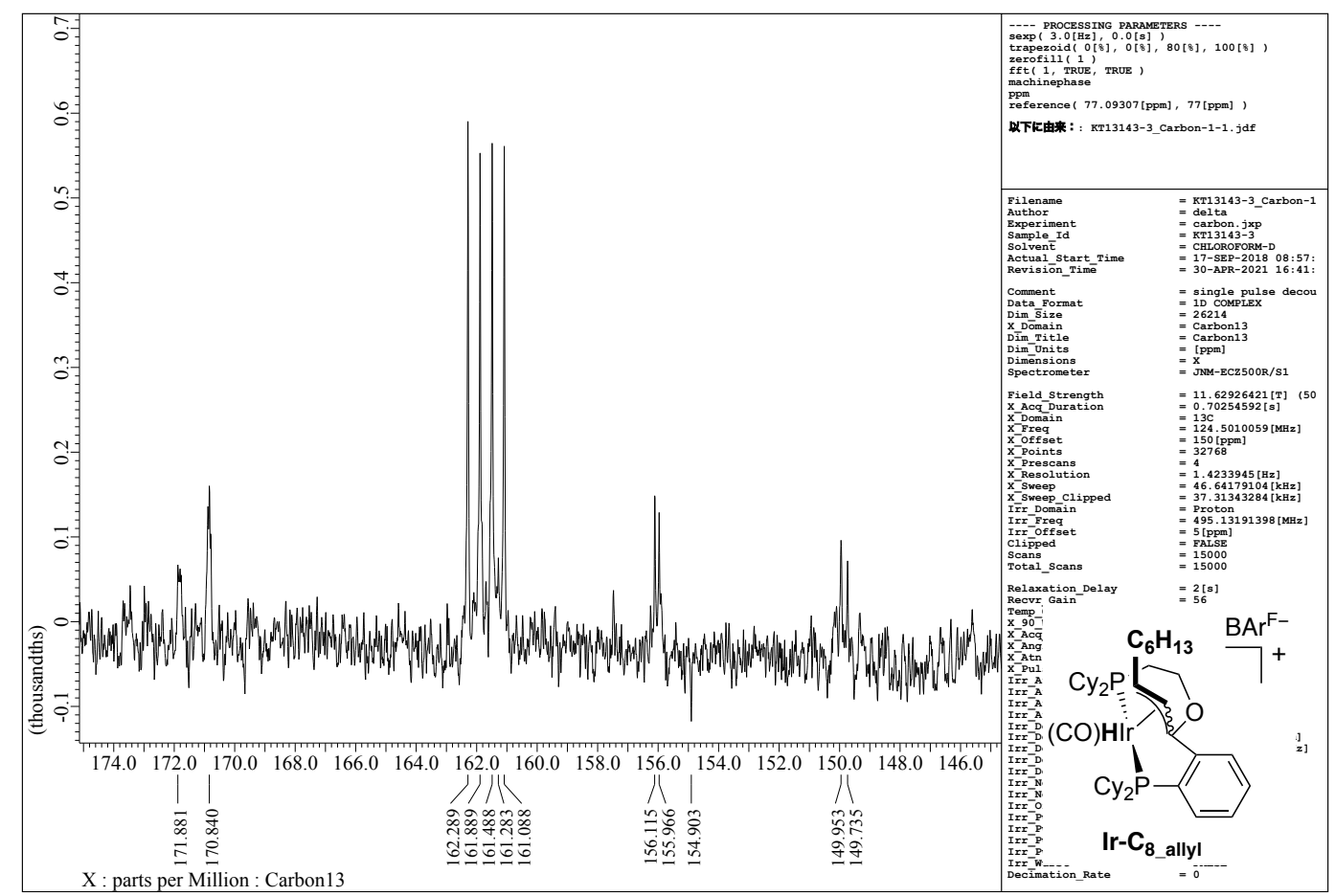

Figure S34. Selected region (175-145 ppm) of ${ }^{13} \mathrm{C}$ NMR spectrum of $\mathbf{I r}-\mathbf{C}_{\mathbf{8} \_ \text {allyl }}$ 


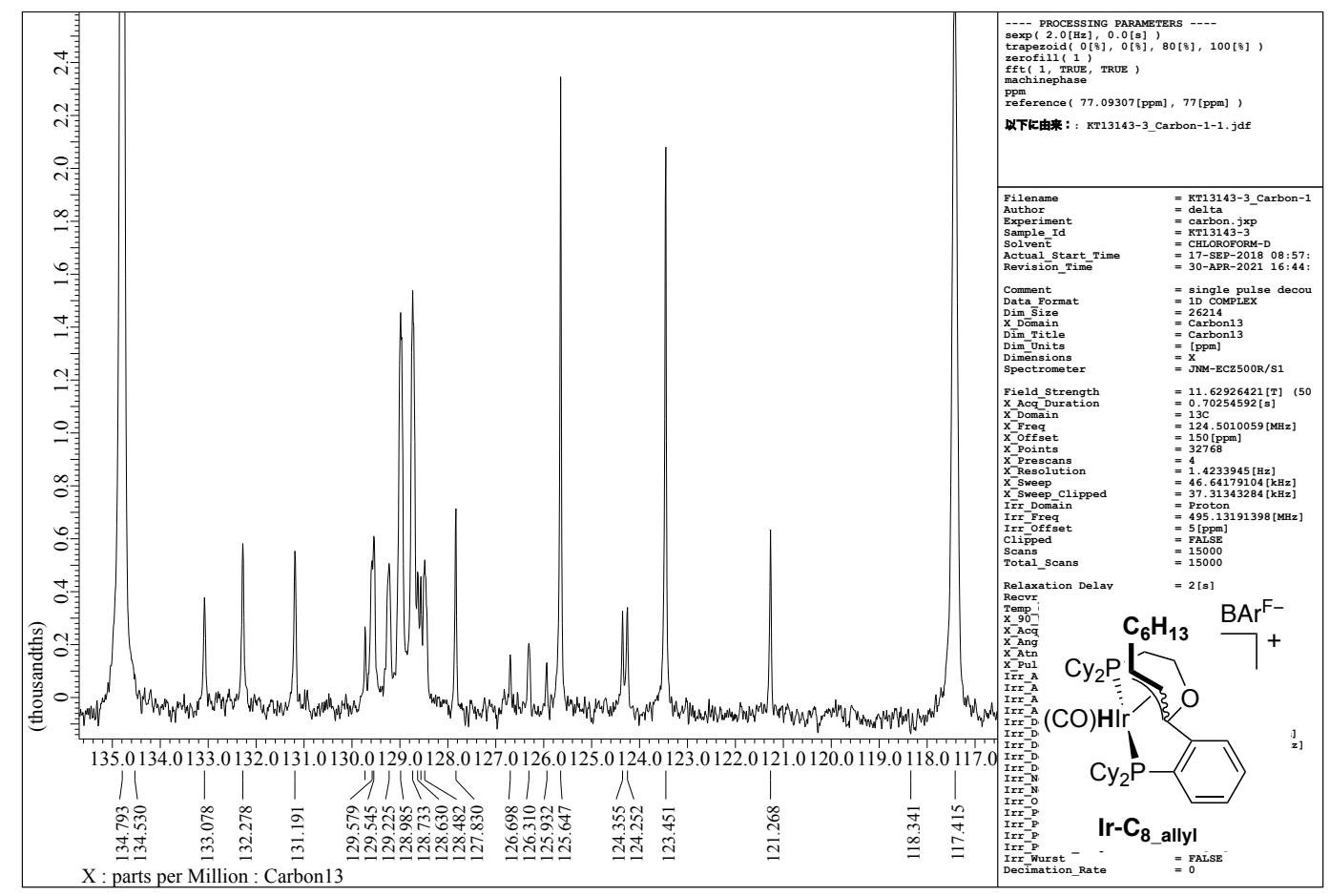

Figure S35. Selected region (136-116 ppm) of ${ }^{13} \mathrm{C}$ NMR spectrum of Ir-C $\mathbf{C}_{\mathbf{8} \_ \text {allyl }}$

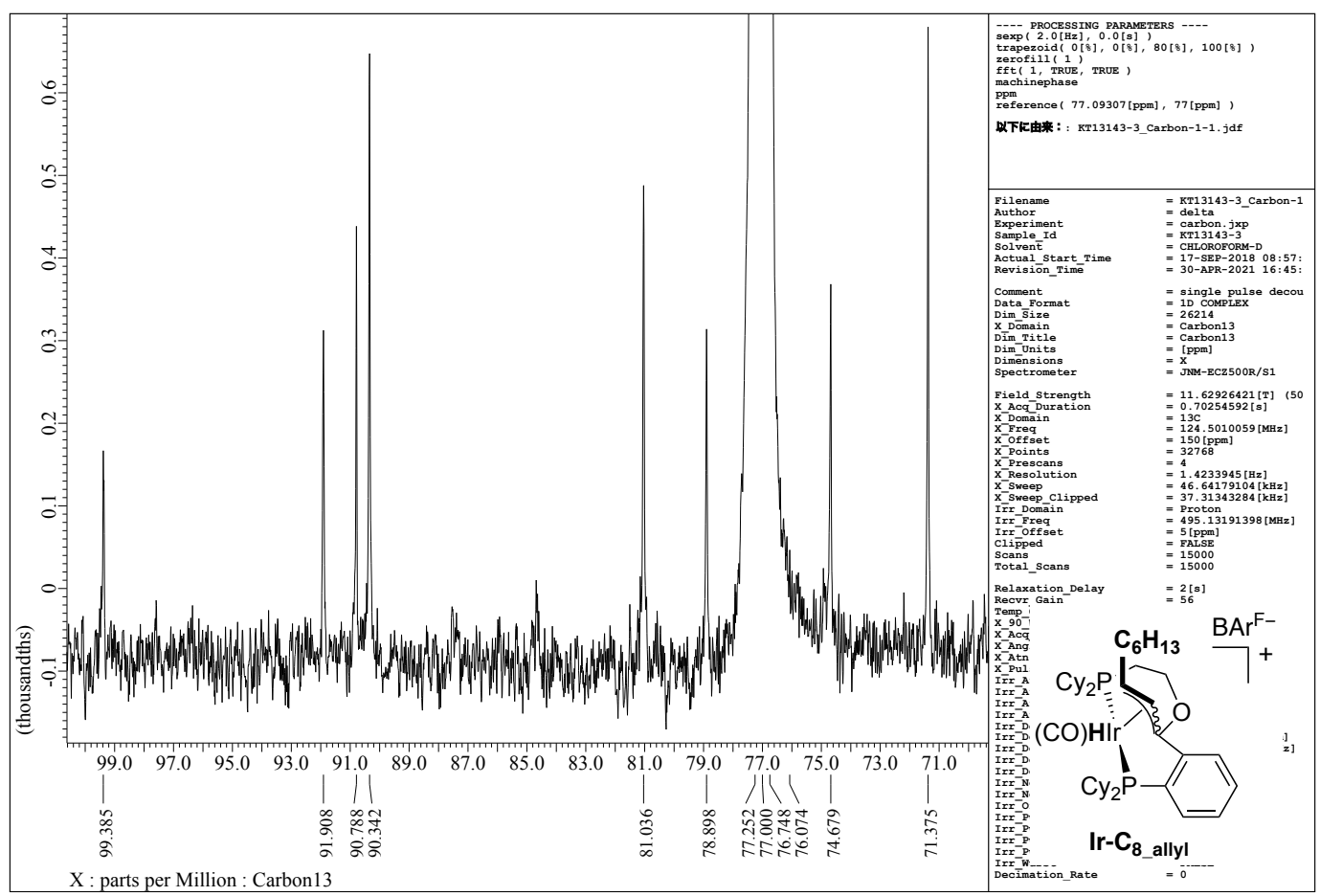

Figure S36. Selected region (100-69 ppm) of ${ }^{13} \mathrm{C}$ NMR spectrum of $\mathbf{I r}-\mathbf{C}_{\mathbf{8}_{-} \text {allyl }}$ 


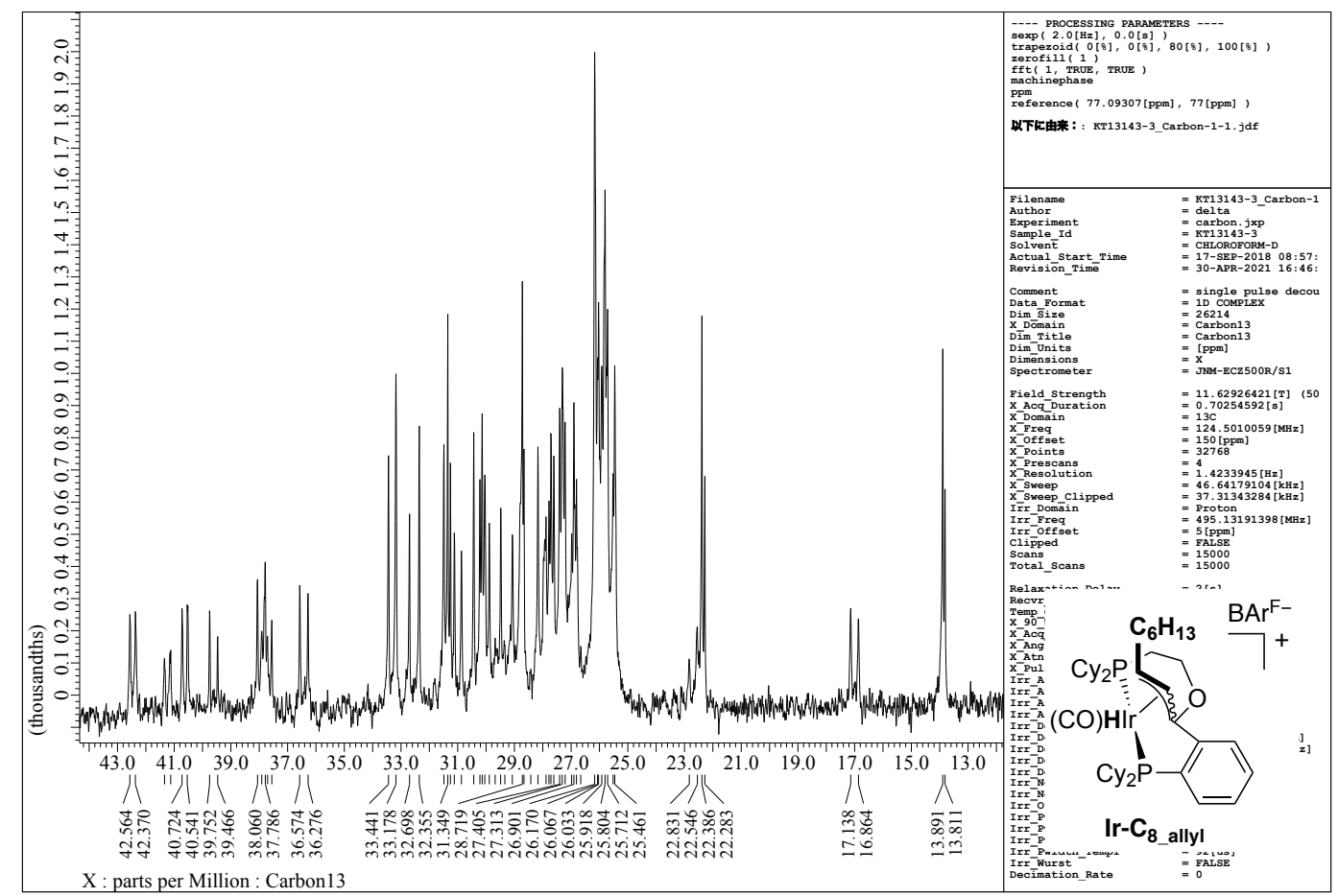

Figure S37. Selected region (44-12 ppm) of ${ }^{13} \mathrm{C}$ NMR spectrum of $\mathbf{I r}-\mathbf{C}_{\boldsymbol{8}_{-} \text {allyl }}$

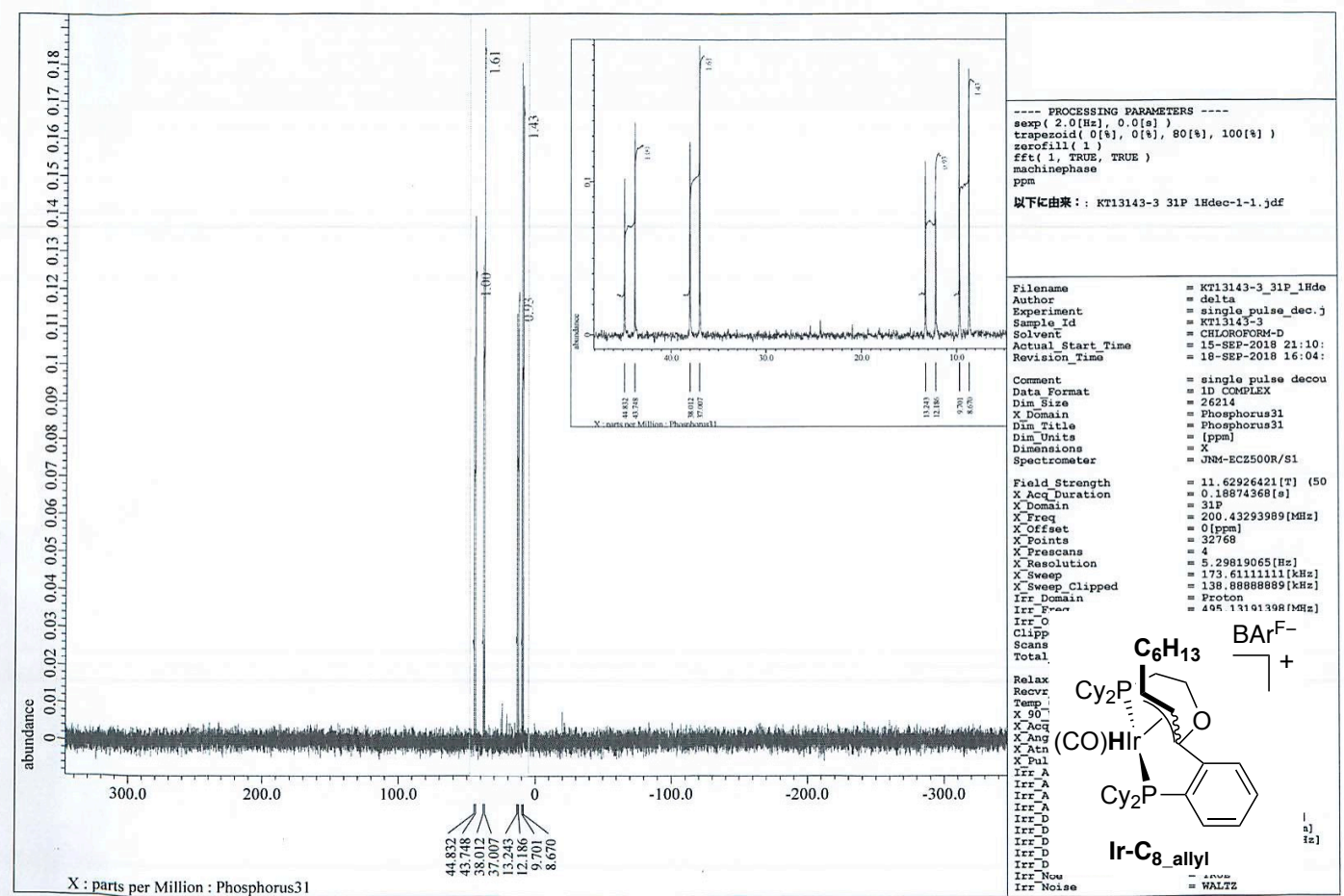

Figure S38. ${ }^{31} \mathrm{P}$ NMR spectrum of $\mathbf{I r}-\mathbf{C}_{\boldsymbol{8}_{-} \text {allyl }}$ 


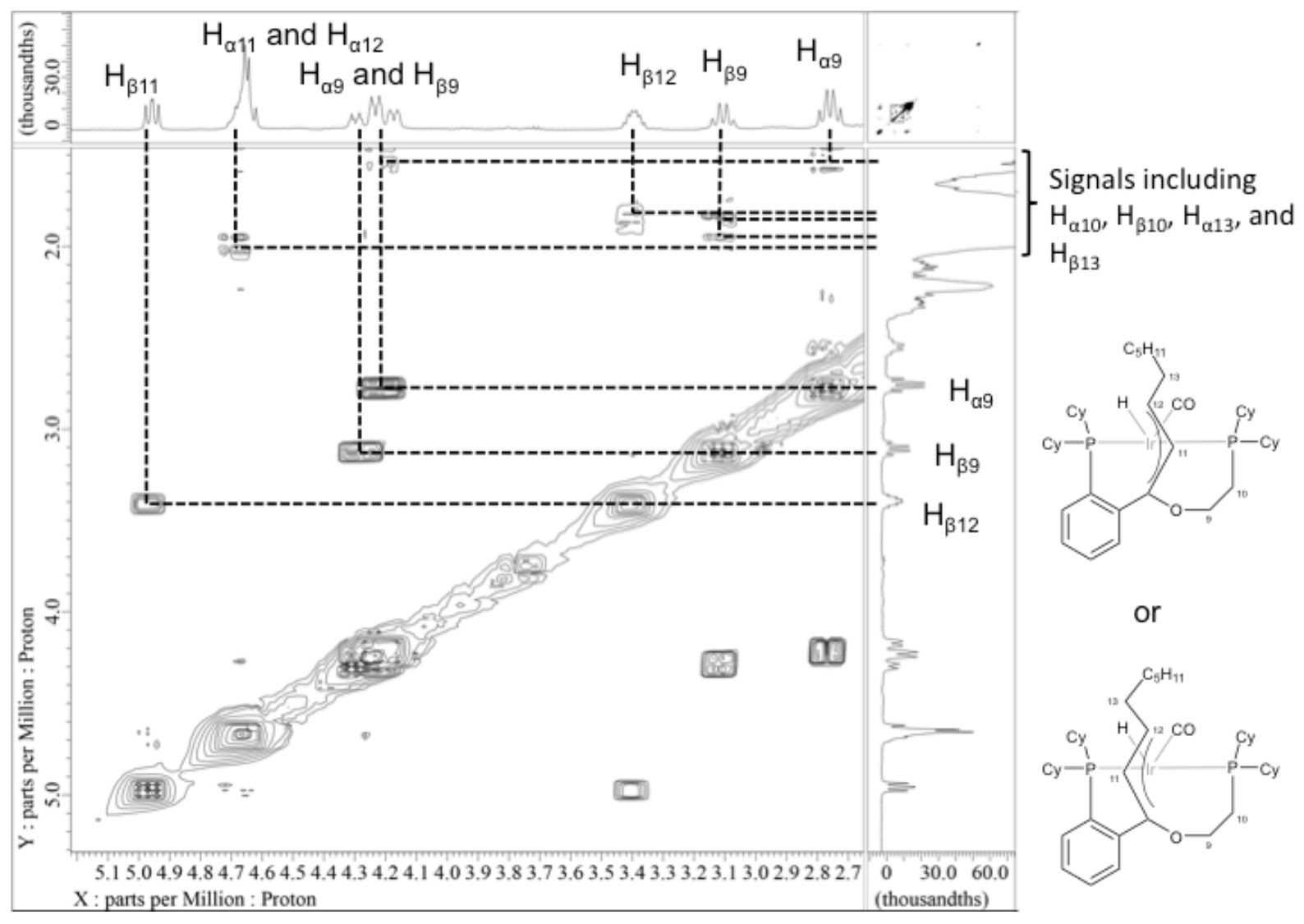

Figure S39. Selected region of HHCOSY spectrum of $\mathbf{I r}-\mathbf{C}_{\mathbf{8}_{-} \text {allyl }}$

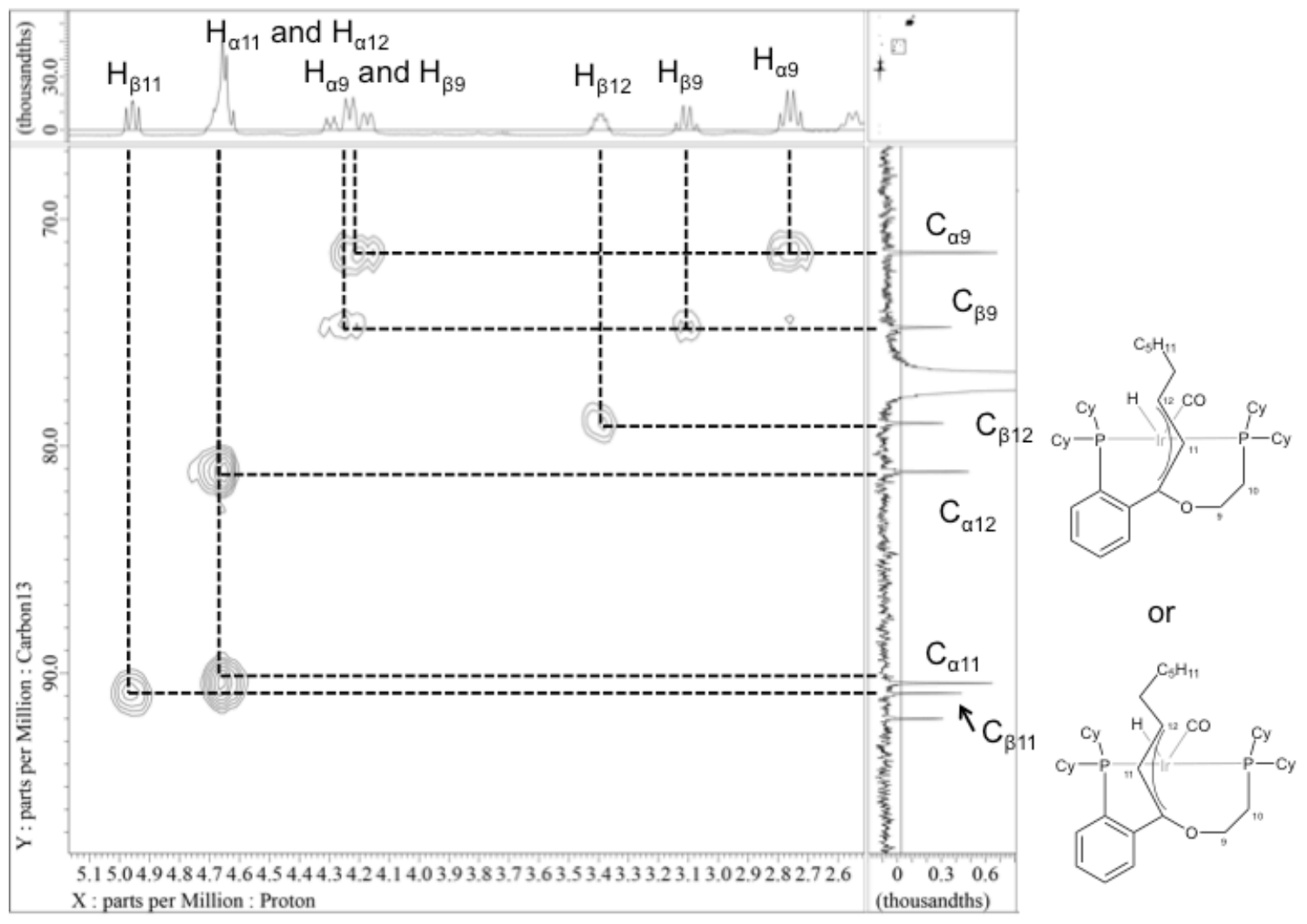

Figure S40. Selected region of HMQC spectrum of $\mathbf{I r}-\mathbf{C}_{\mathbf{8} \_ \text {allyl }}$ 

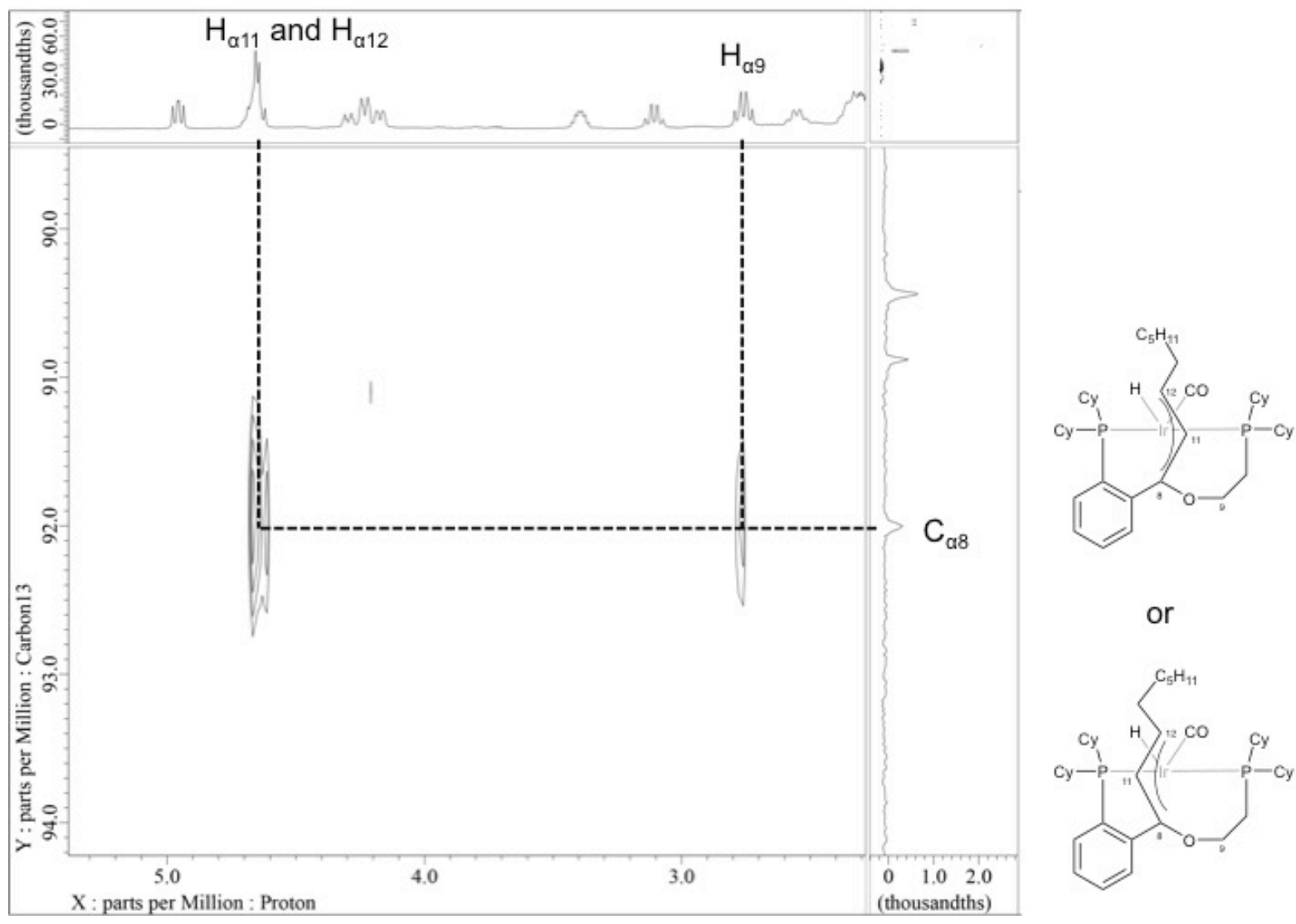

Figure S41. Selected region-1 of HMBC spectrum of $\mathbf{I r - \mathbf { C } _ { \mathbf { 8 } } \text { allyl }}$
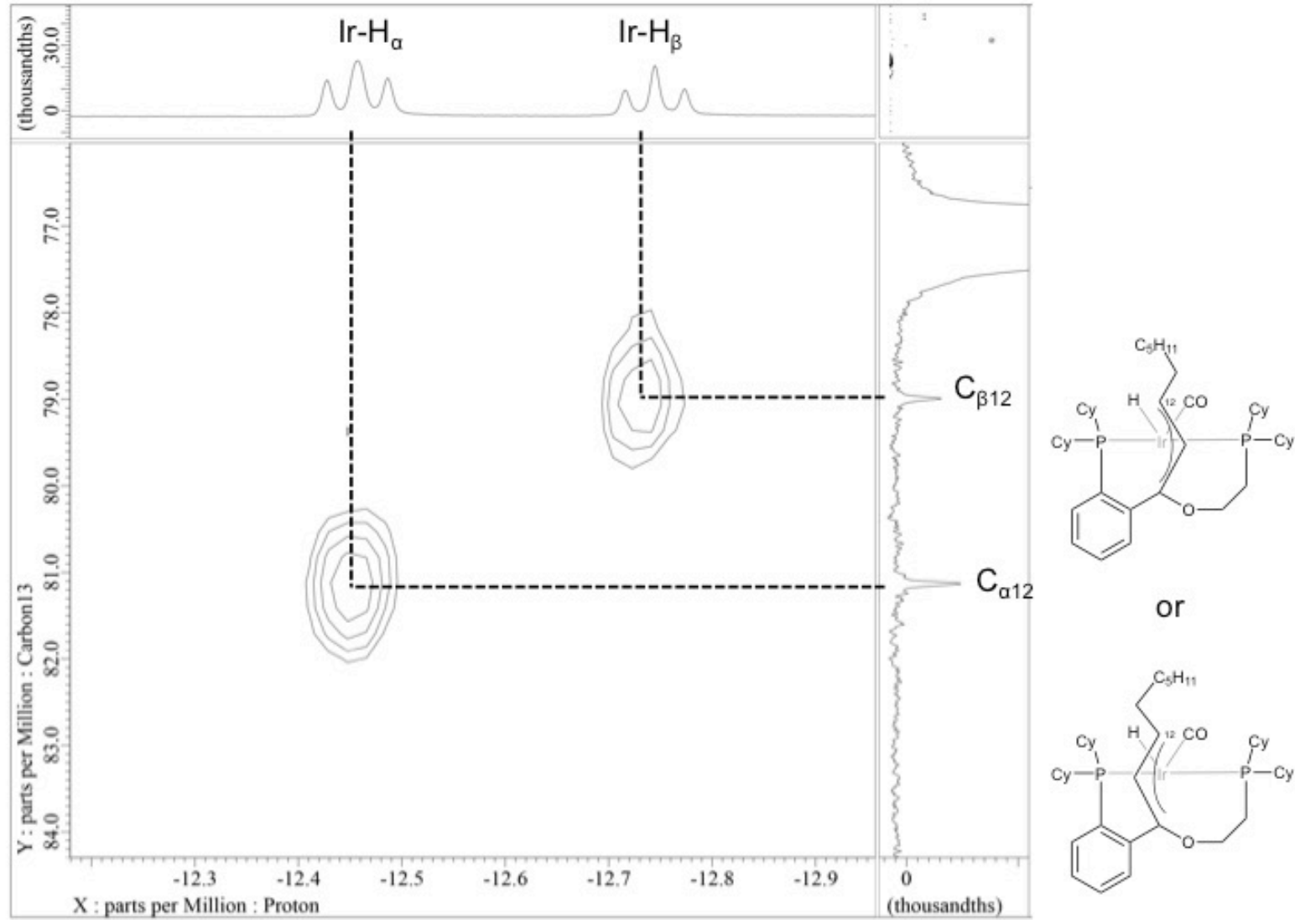

$\mathrm{C}_{a 12}$

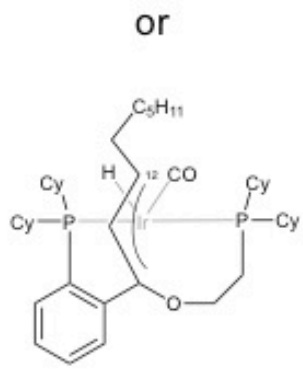

Figure $\mathbf{S 4 2}$. Selected region-2 of HMBC spectrum of $\mathbf{I r}-\mathbf{C}_{\mathbf{8} \_ \text {allyl }}$ 

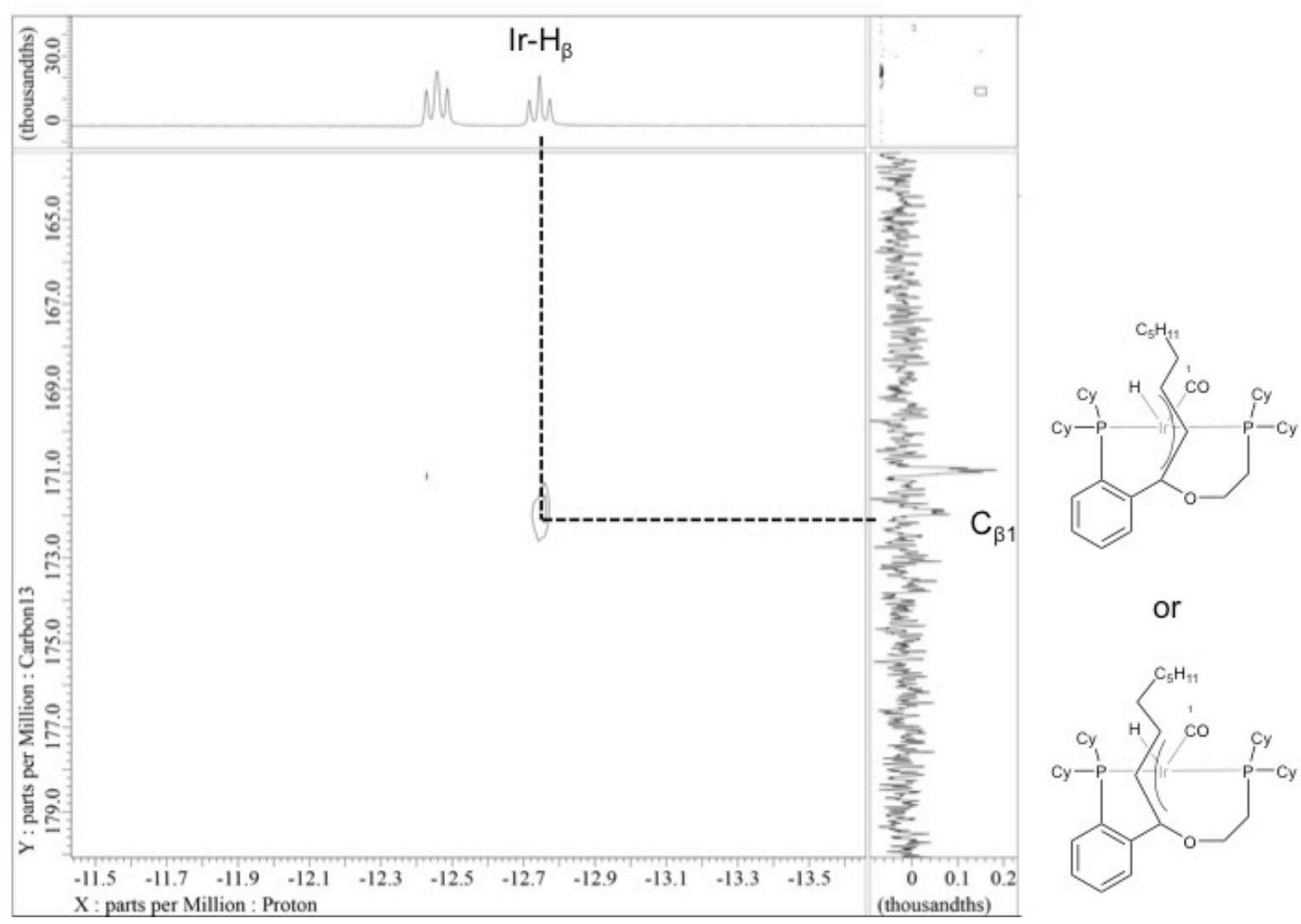

or

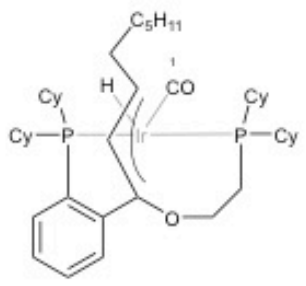

Figure S43. Selected region-3 of HMBC spectrum of $\mathbf{I r - C _ { 8 } \text { ally1 }}$

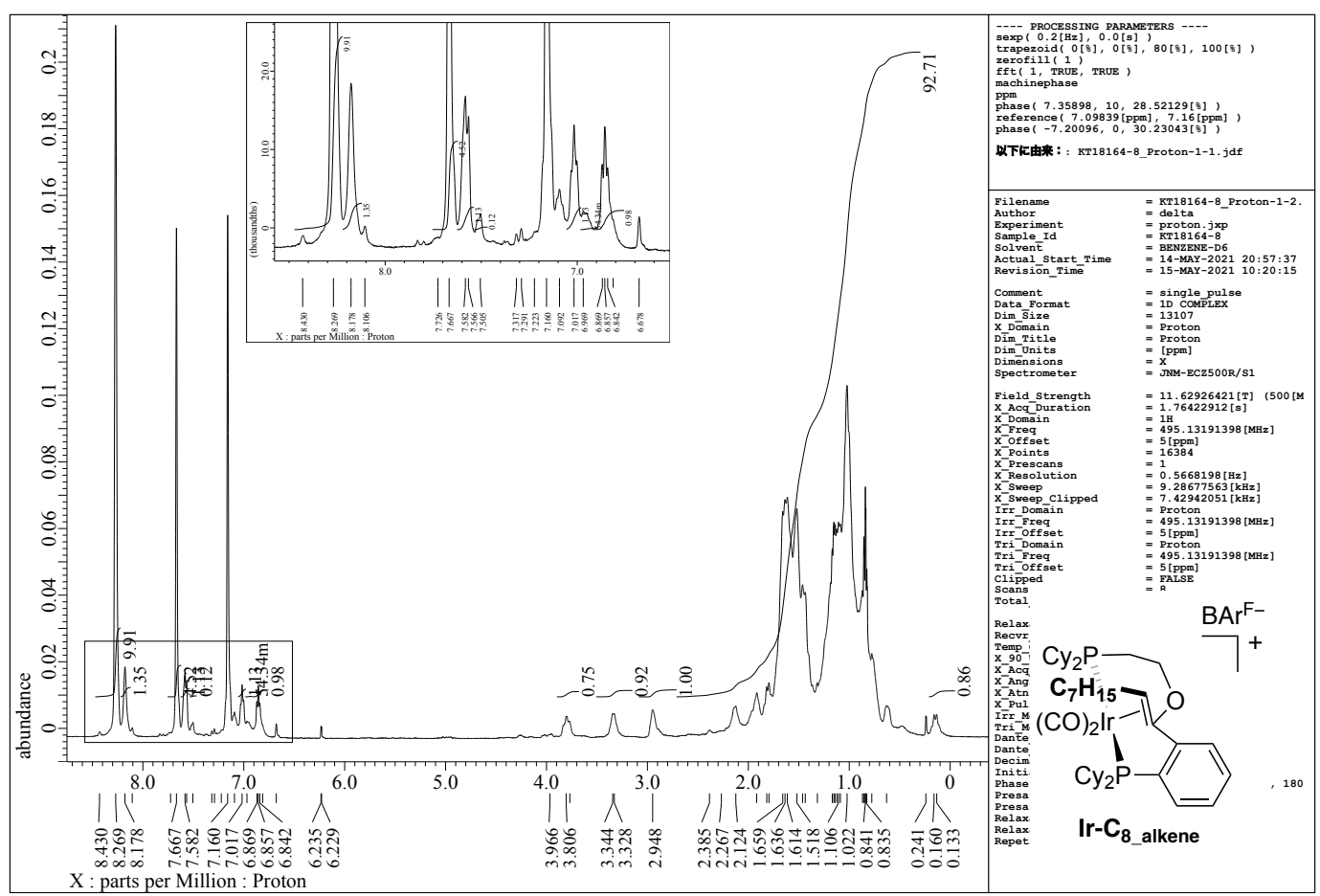

Figure S44. ${ }^{1} \mathrm{H}$ NMR spectrum of $\mathbf{I r}-\mathbf{C}_{\mathbf{8}_{-} \text {alkene }}$ 


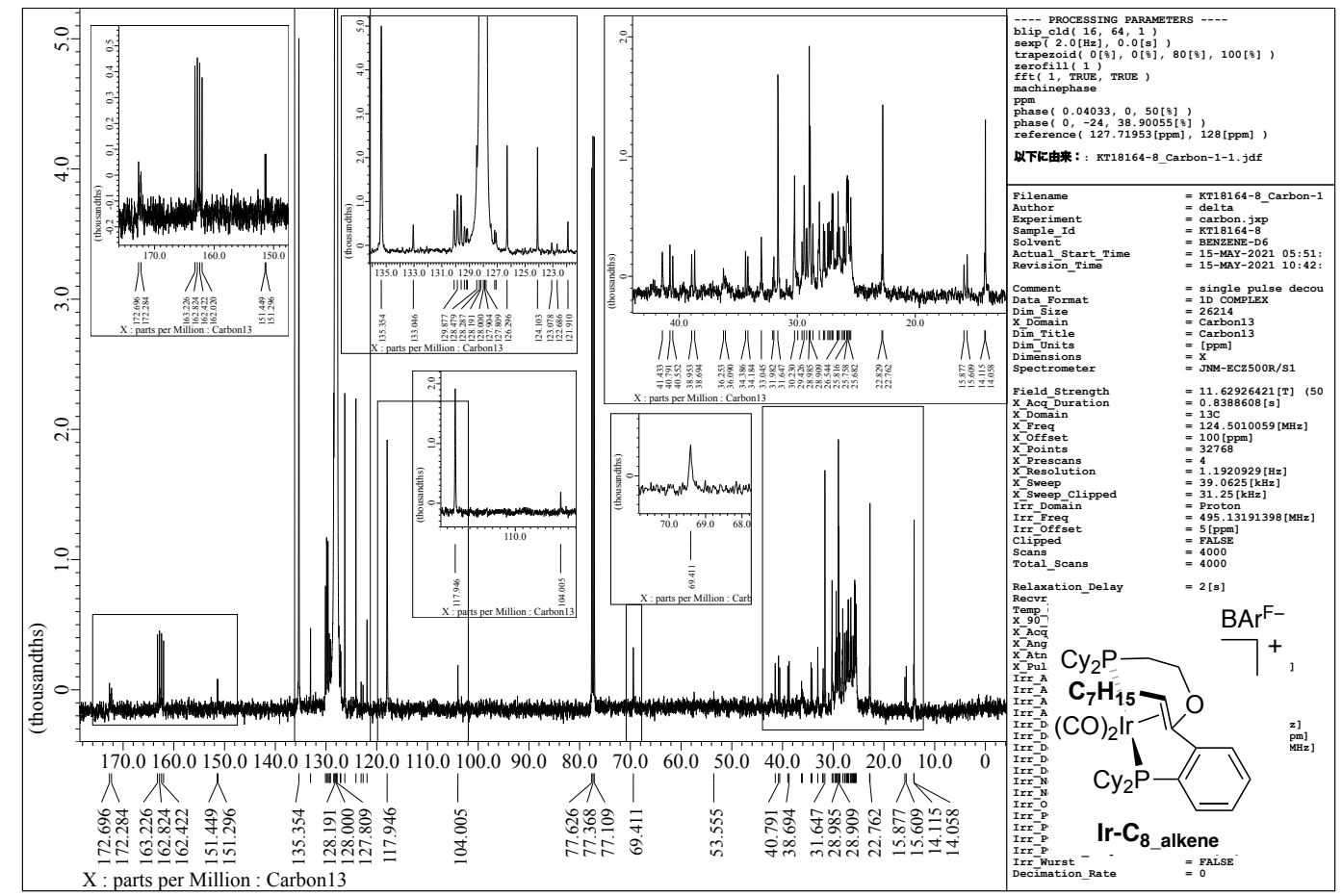

Figure S45. ${ }^{13} \mathrm{C}$ NMR spectrum of $\mathbf{I r}-\mathbf{C}_{8_{-} \text {alkene }}$

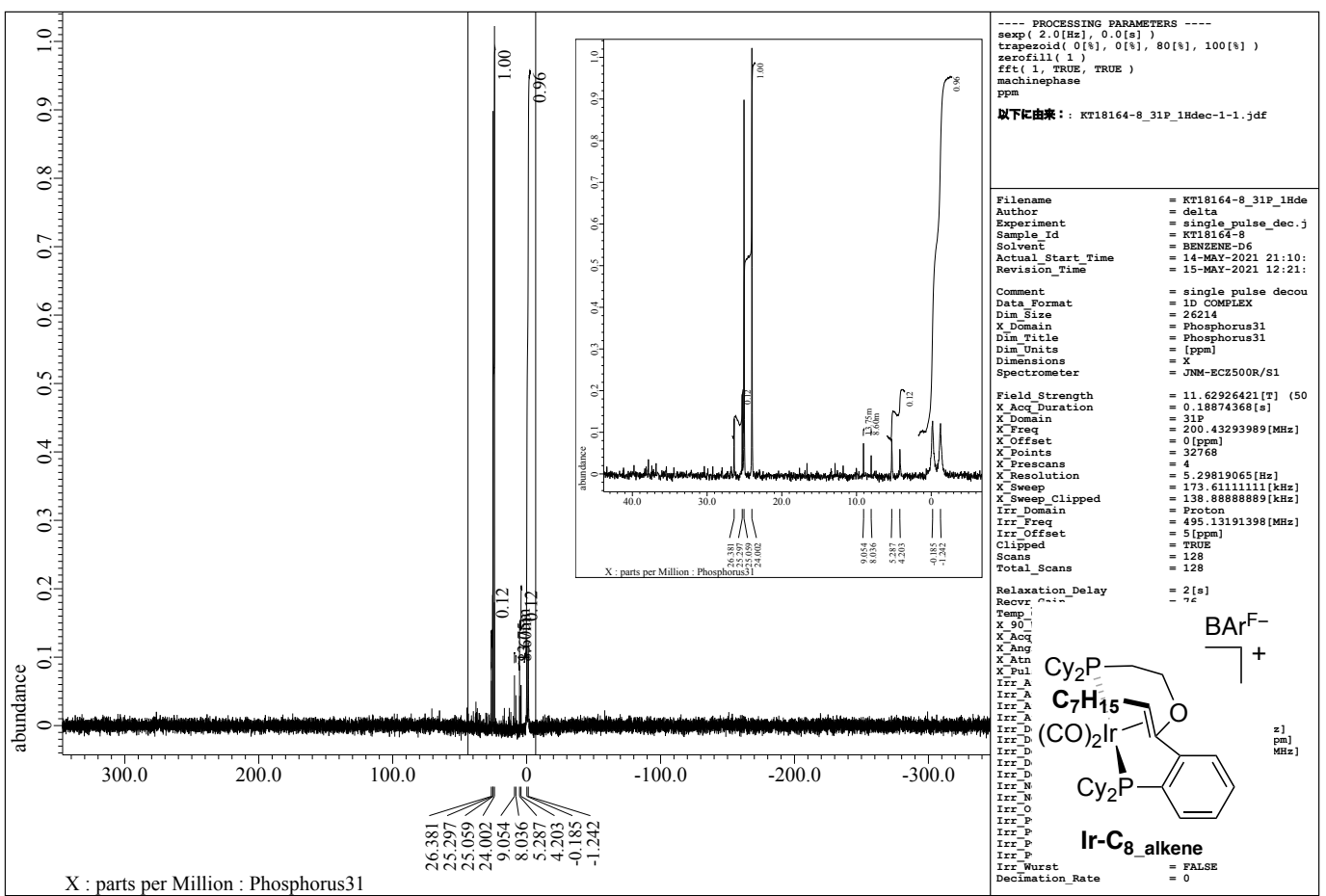

Figure S46. ${ }^{31} \mathrm{P}$ NMR spectrum of Ir-C $\mathbf{C}_{\mathbf{8} \_ \text {alkene }}$ 


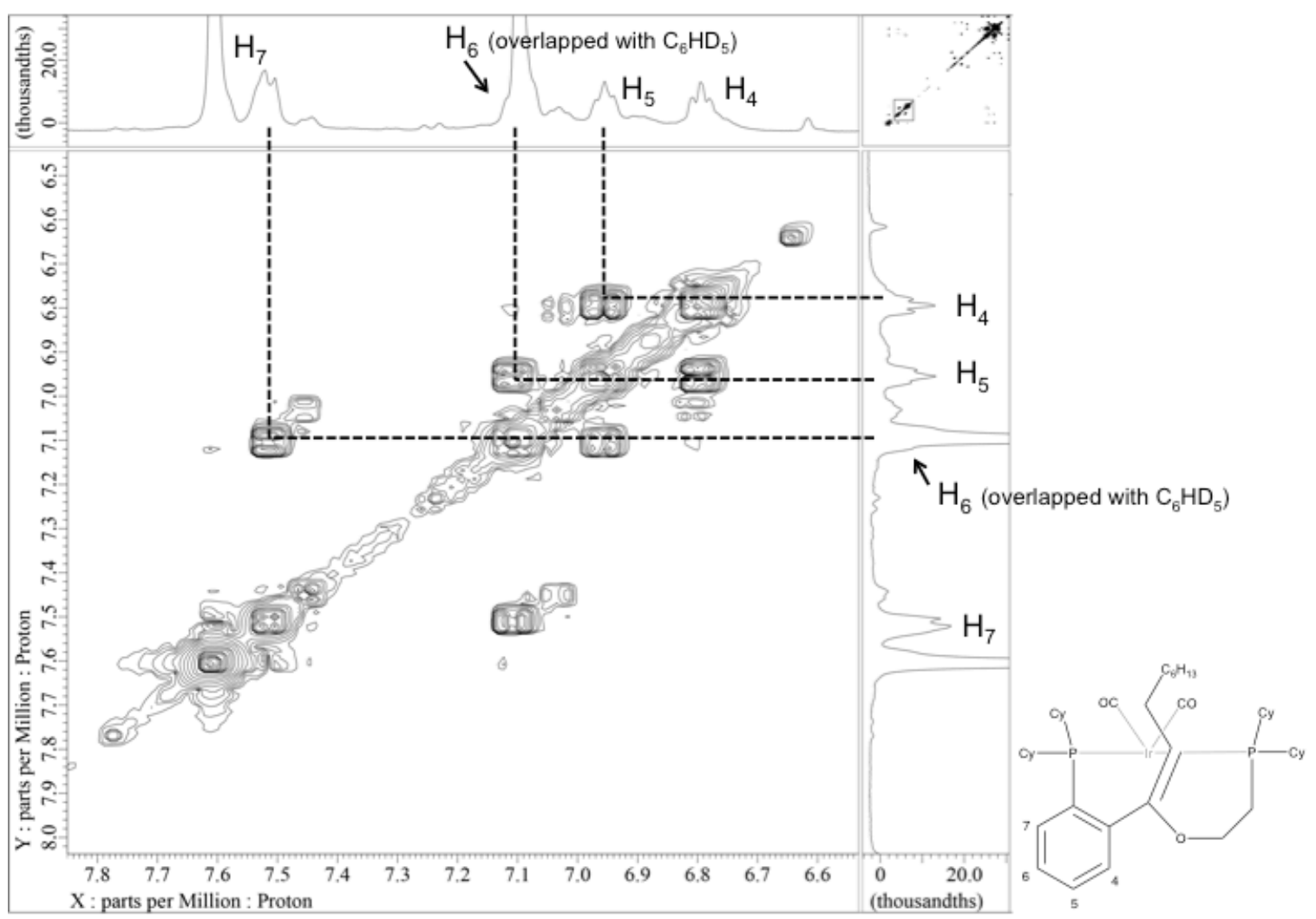

Figure S47. Selected region-1 of HHCOSY spectrum of $\mathbf{I r - \mathbf { C } _ { \mathbf { 8 } _ { - } \text { alkene } }}$

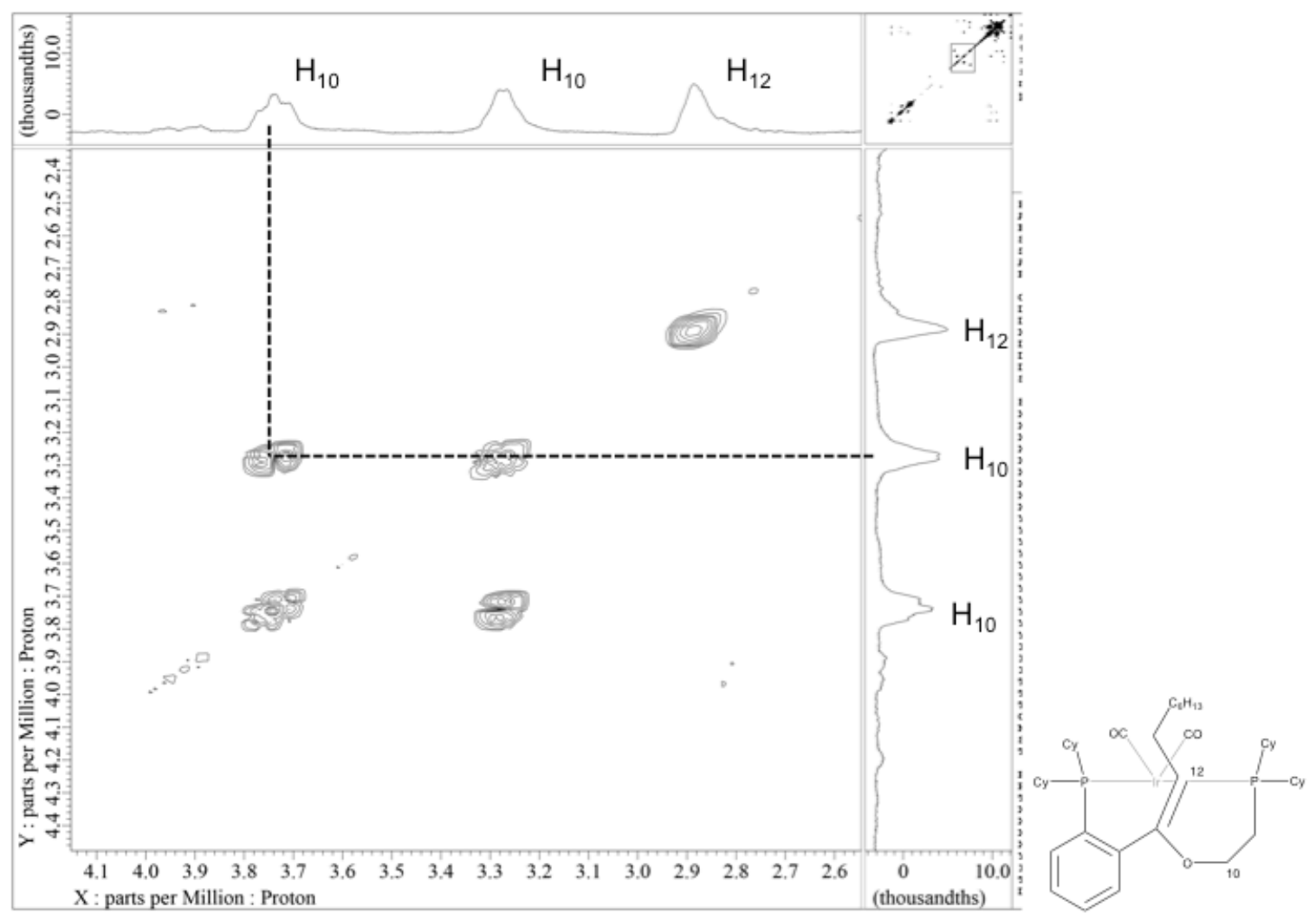

Figure S48. Selected region-2 of HHCOSY spectrum of Ir-C $\mathbf{8}_{\text {_alkene }}$ 


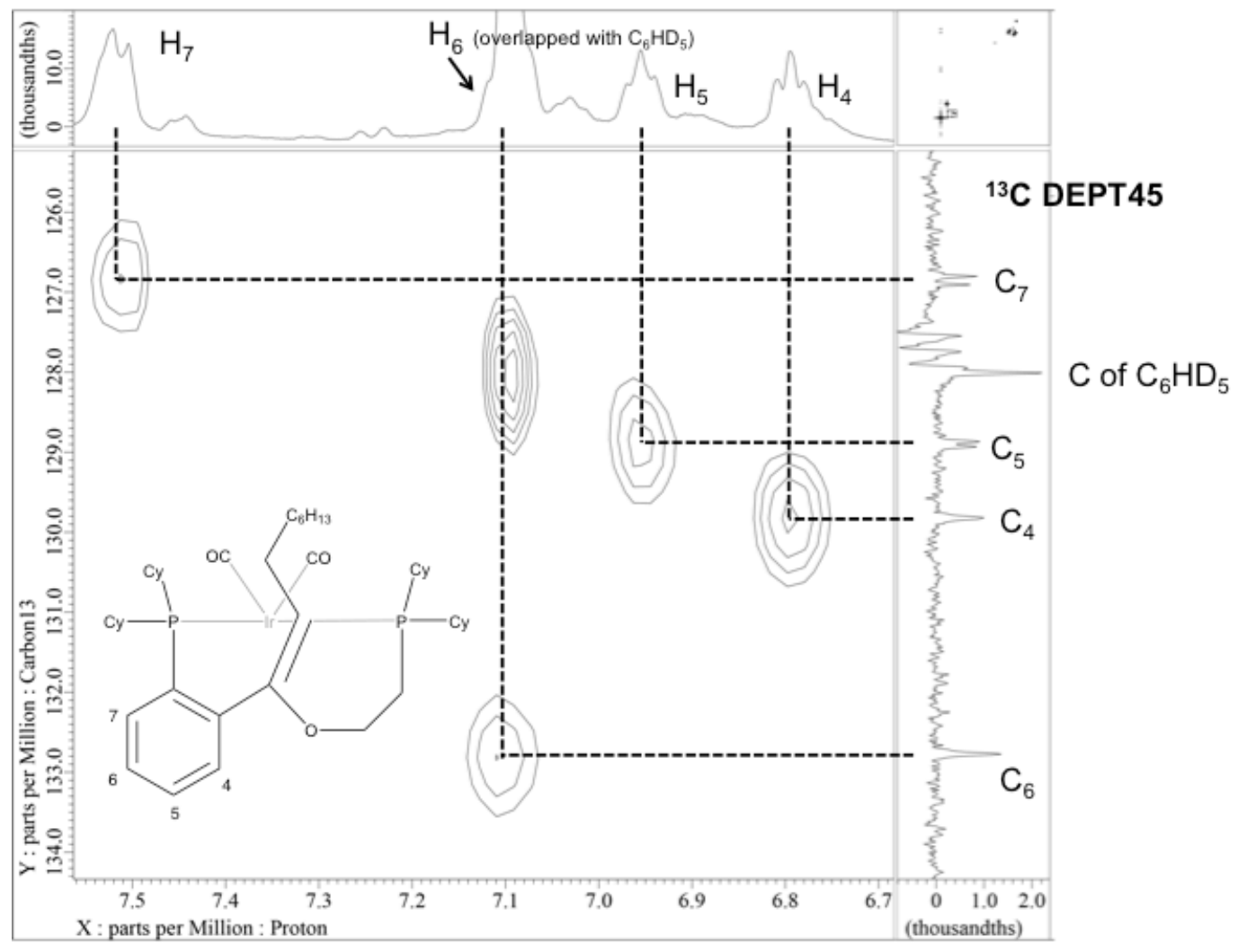

Figure S49. Selected region-1 of HMQC spectrum of $\mathbf{I r - C _ { 8 } \text { alkene }}$

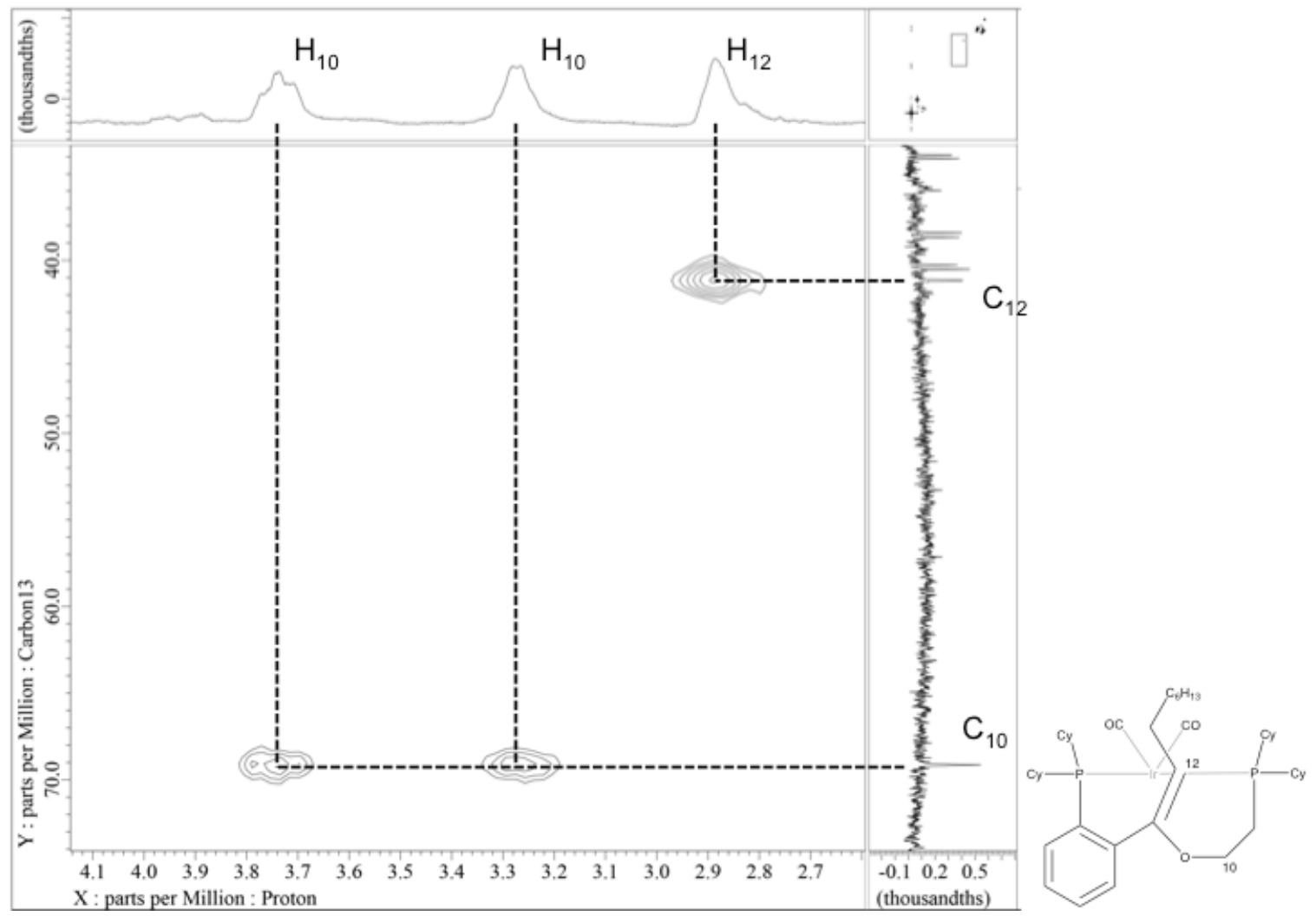

Figure S50. Selected region-2 of HMQC spectrum of $\mathbf{I r - \mathbf { C } _ { \mathbf { 8 } } \text { alkene }}$ 


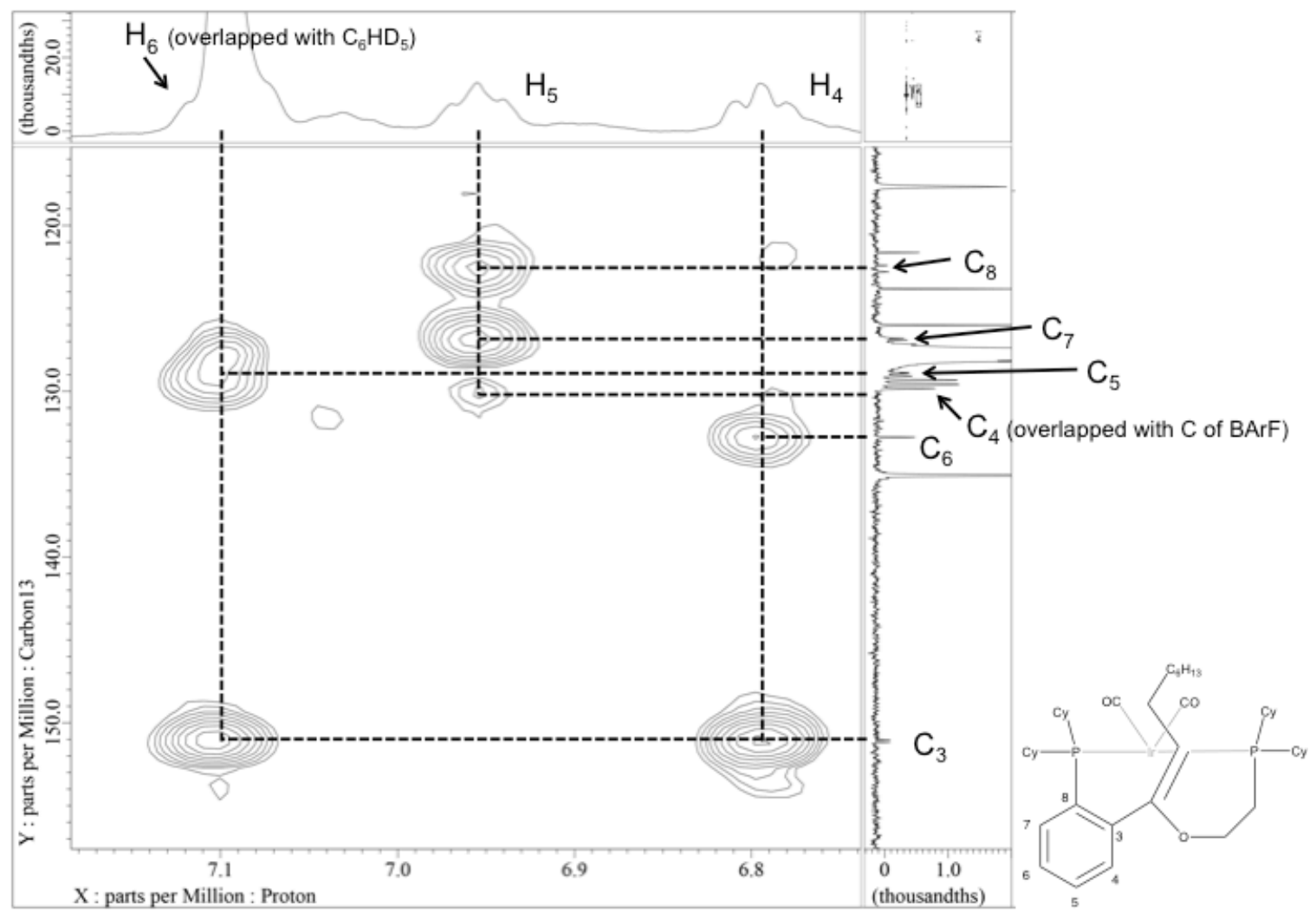

Figure S51. Selected region-1 of HMBC spectrum of Ir-C $\mathbf{8}_{\mathbf{8}}$ alkene

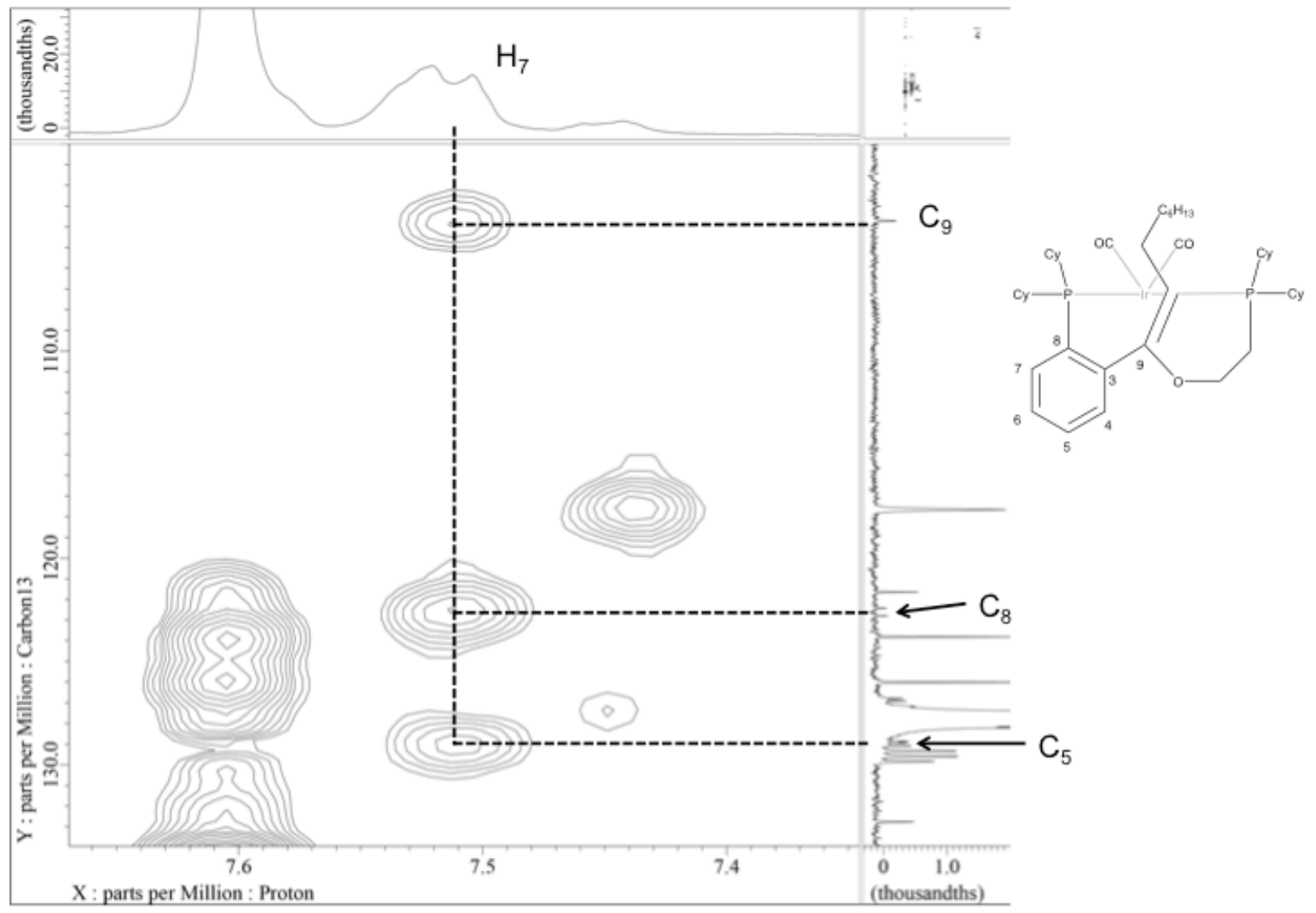

Figure S52. Selected region-2 of HMBC spectrum of $\mathbf{I r}-\mathbf{C}_{\boldsymbol{8}_{-} \text {alkene }}$ 


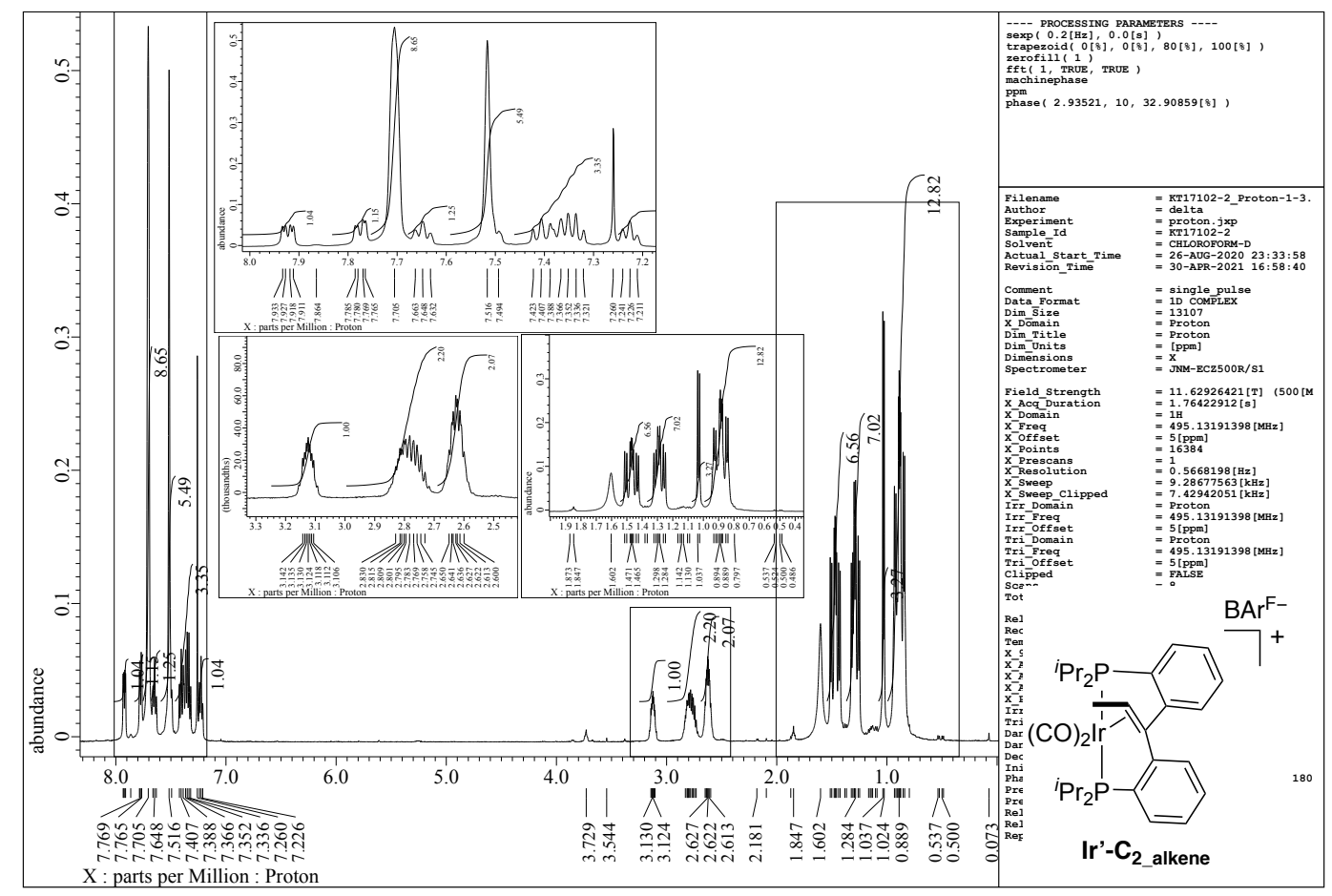

Figure S53. ${ }^{1} \mathrm{H}$ NMR spectrum of Ir'-C 2_alkene

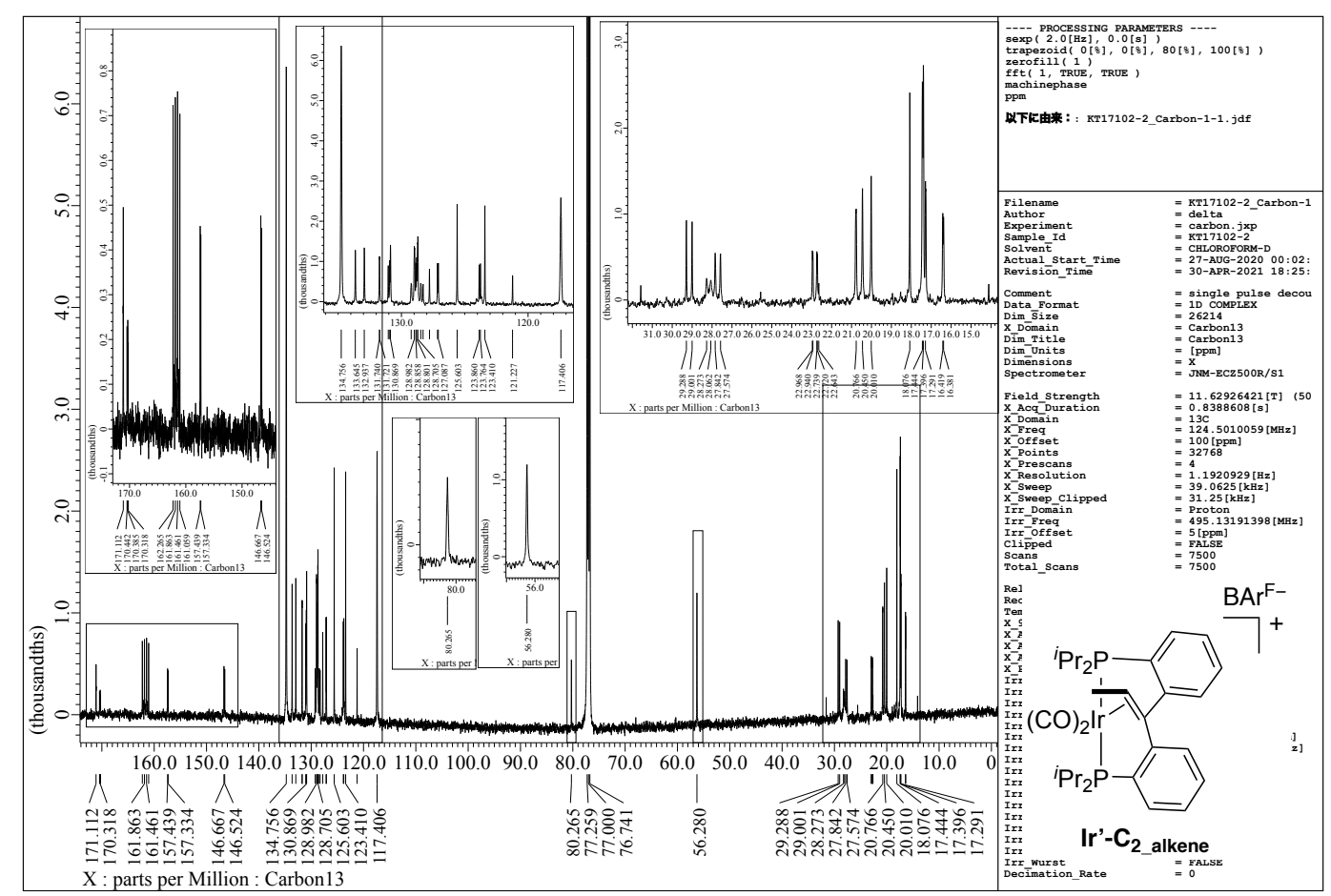

Figure S54. ${ }^{13} \mathrm{C}$ NMR spectrum of Ir'- $\mathbf{C}_{\mathbf{2} \_ \text {alkene }}$ 


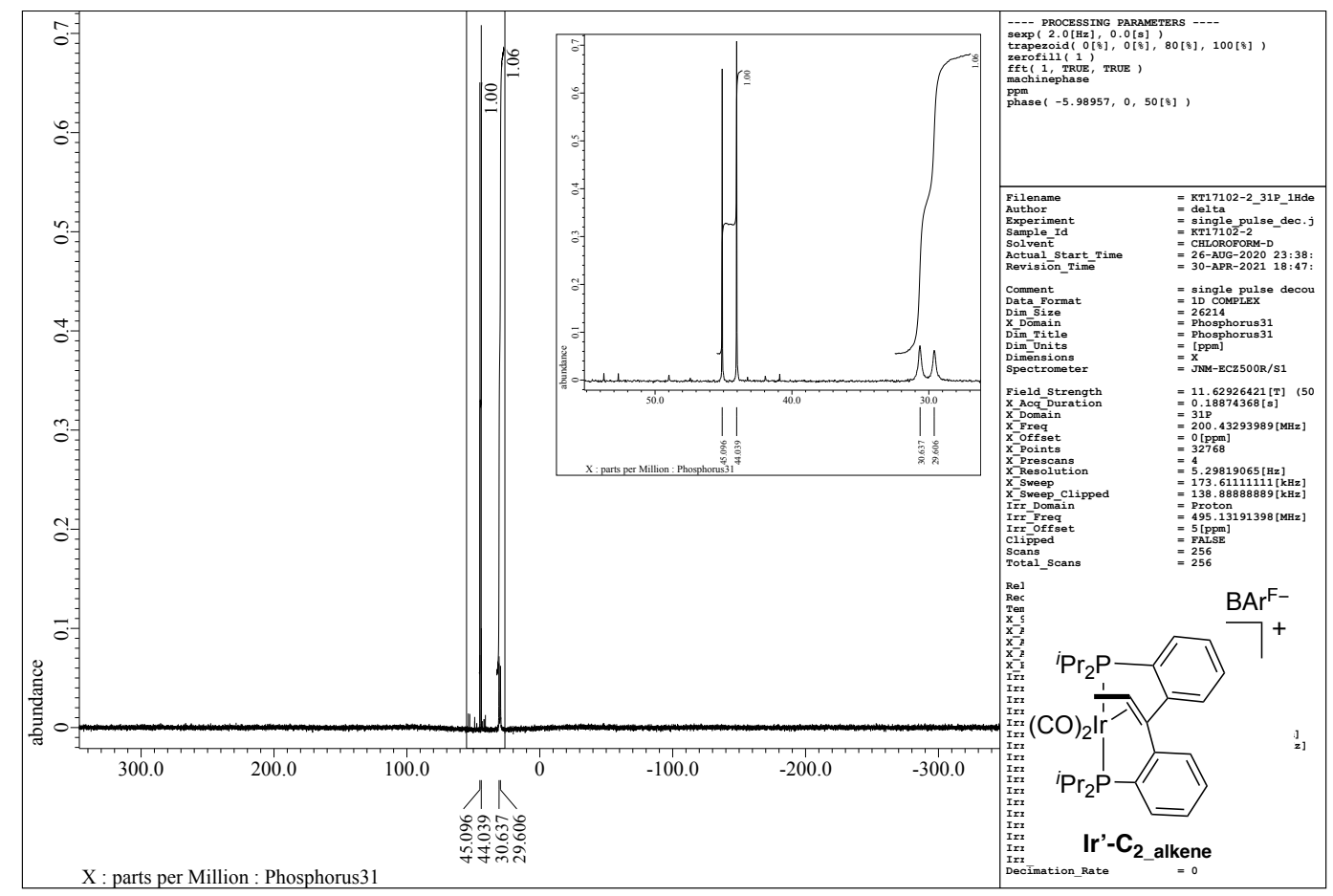

Figure S55. ${ }^{31} \mathrm{P}$ NMR spectrum of Ir'-C $\mathbf{C}_{2}$ alkene

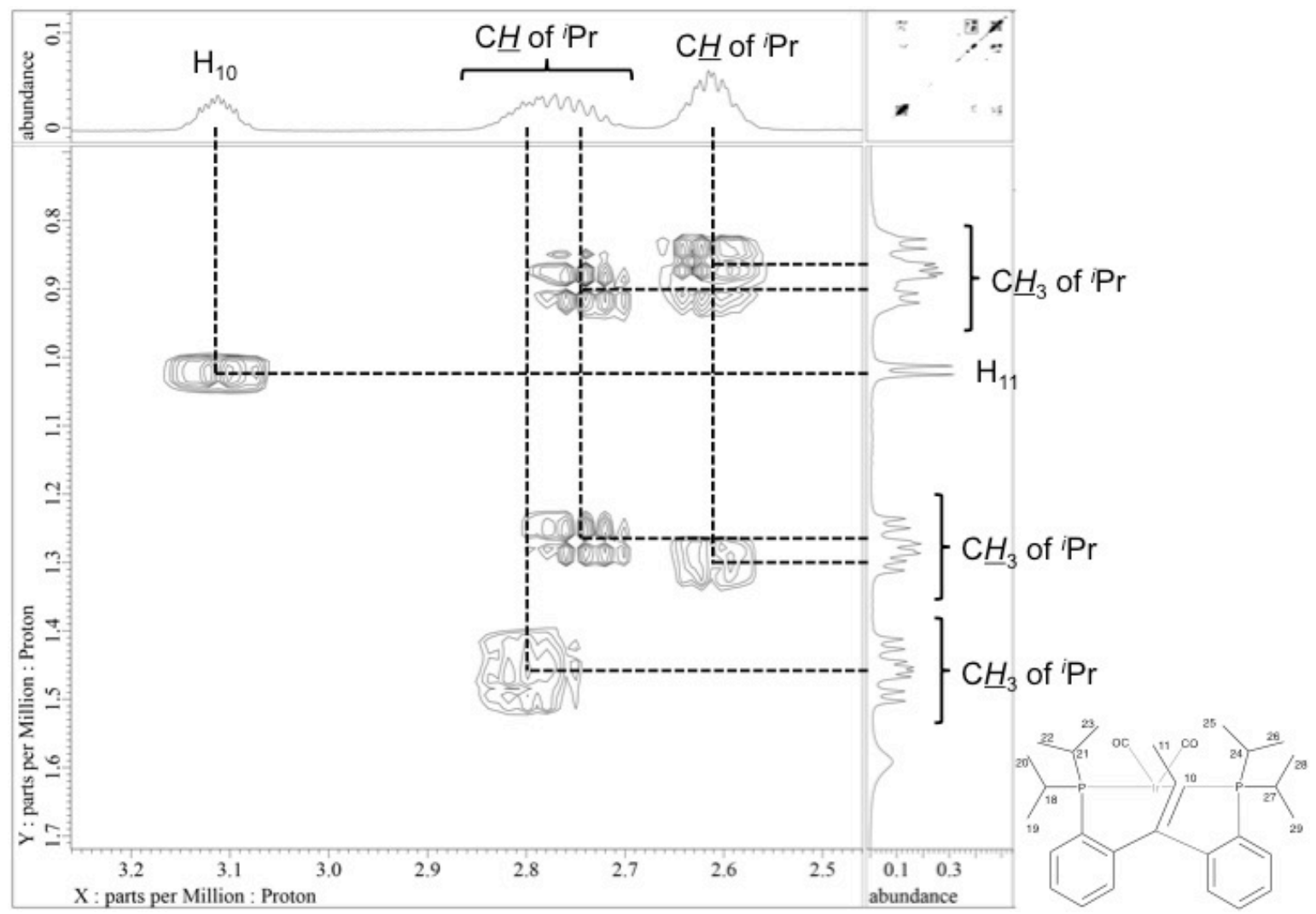

Figure S56. Selected region of HHCOSY spectrum of Ir'-C $\mathbf{2}_{\mathbf{2}}$ alkene 


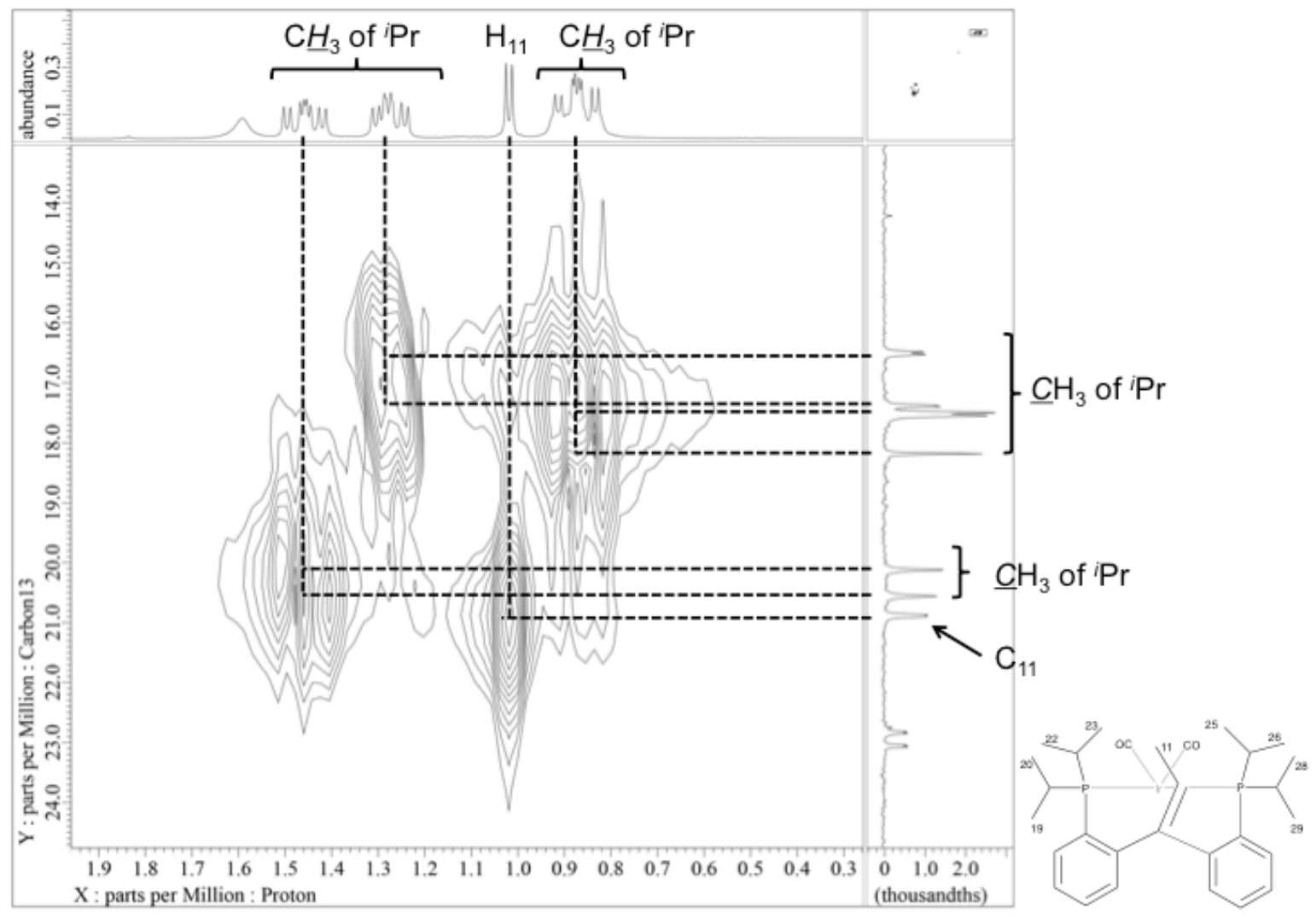

Figure S57. Selected region-1 of HMQC spectrum of Ir'-C 2_alkene

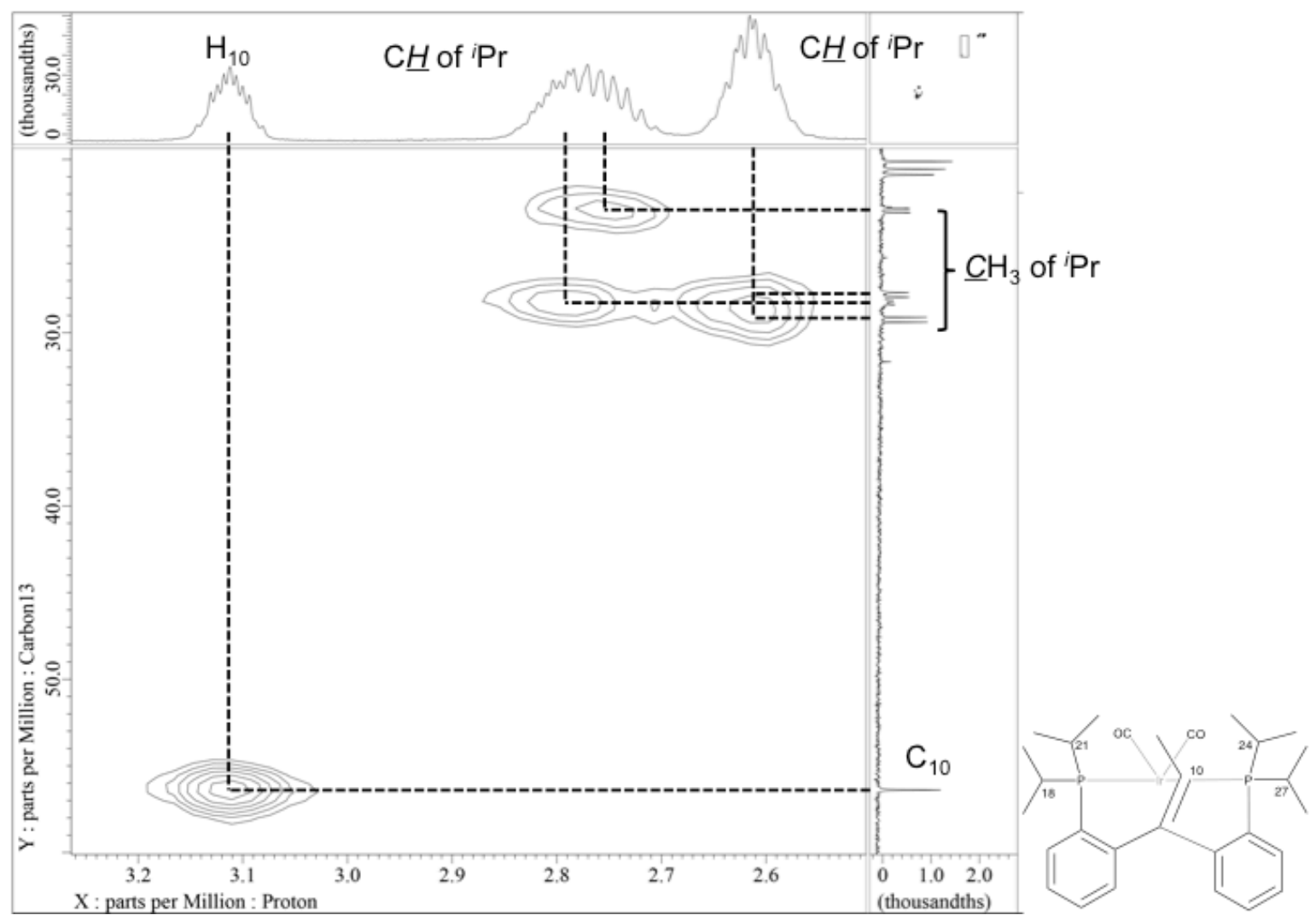

Figure S58. Selected region-2 of HMQC spectrum of Ir'-C 2_alkene 


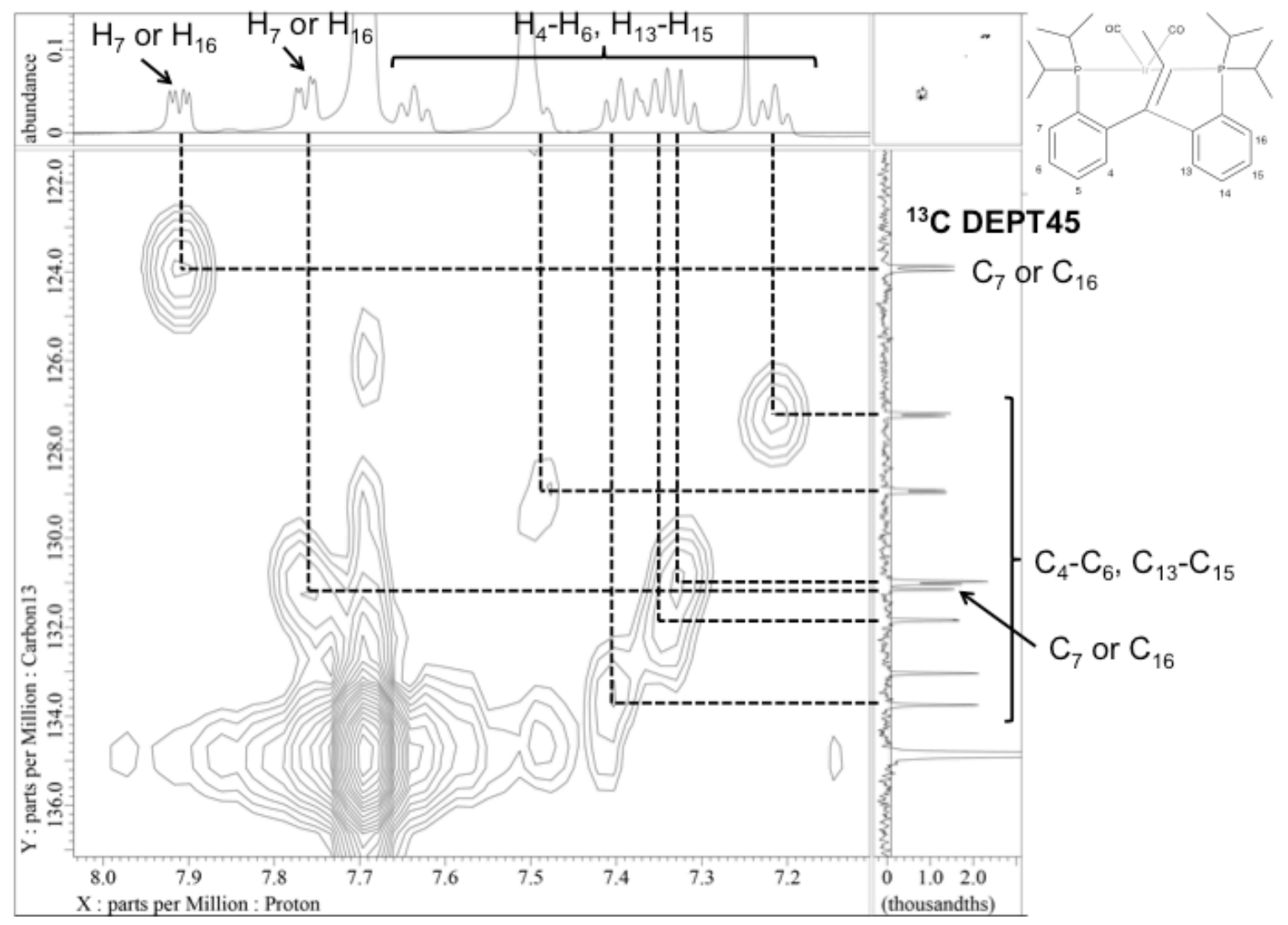

Figure S59. Selected region-3 of HMQC spectrum of Ir'-C 2_alkene

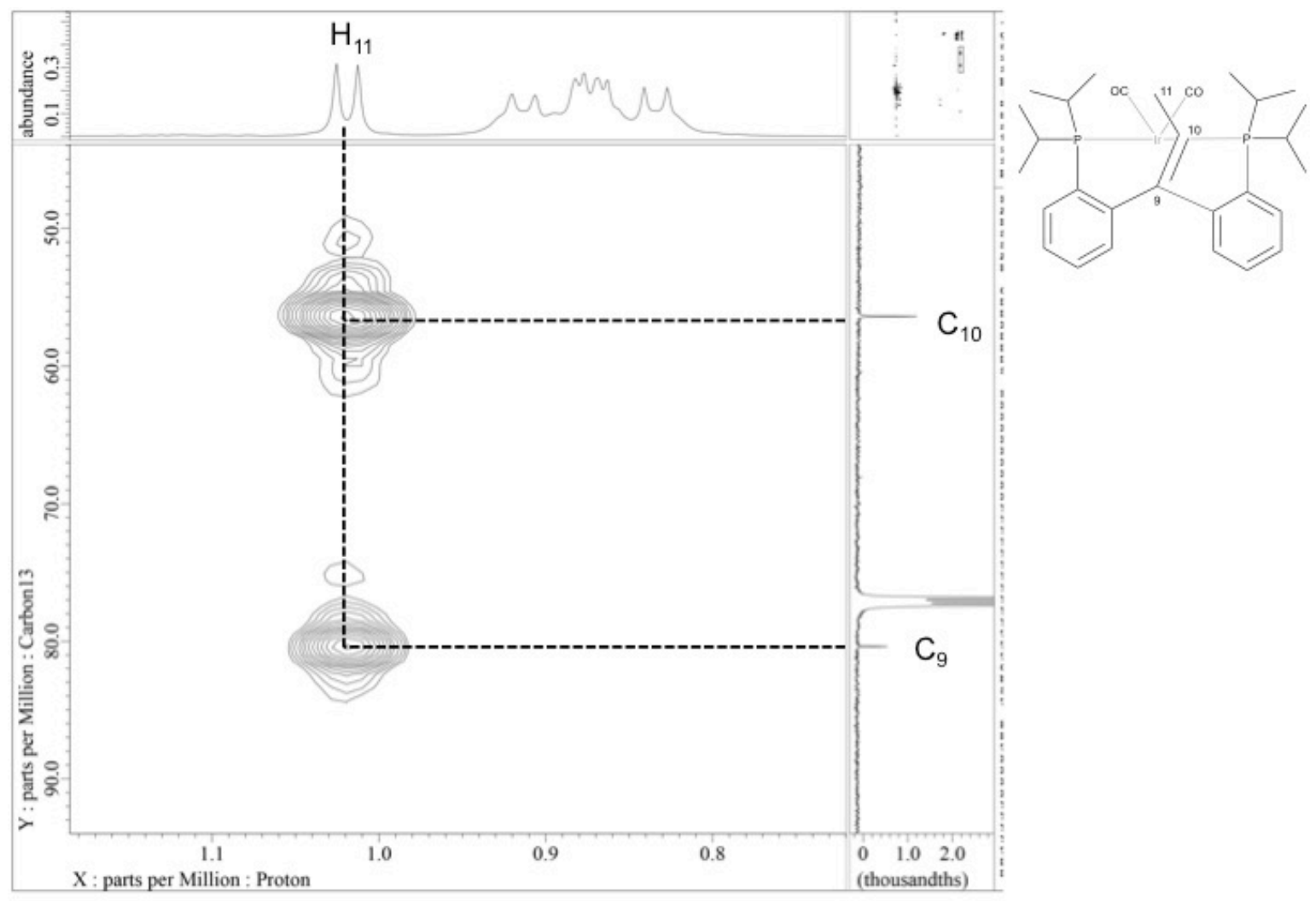

Figure S60. Selected region-1 of HMBC spectrum of Ir'- $\mathbf{C}_{\mathbf{2}_{-} \text {alkene }}$ 


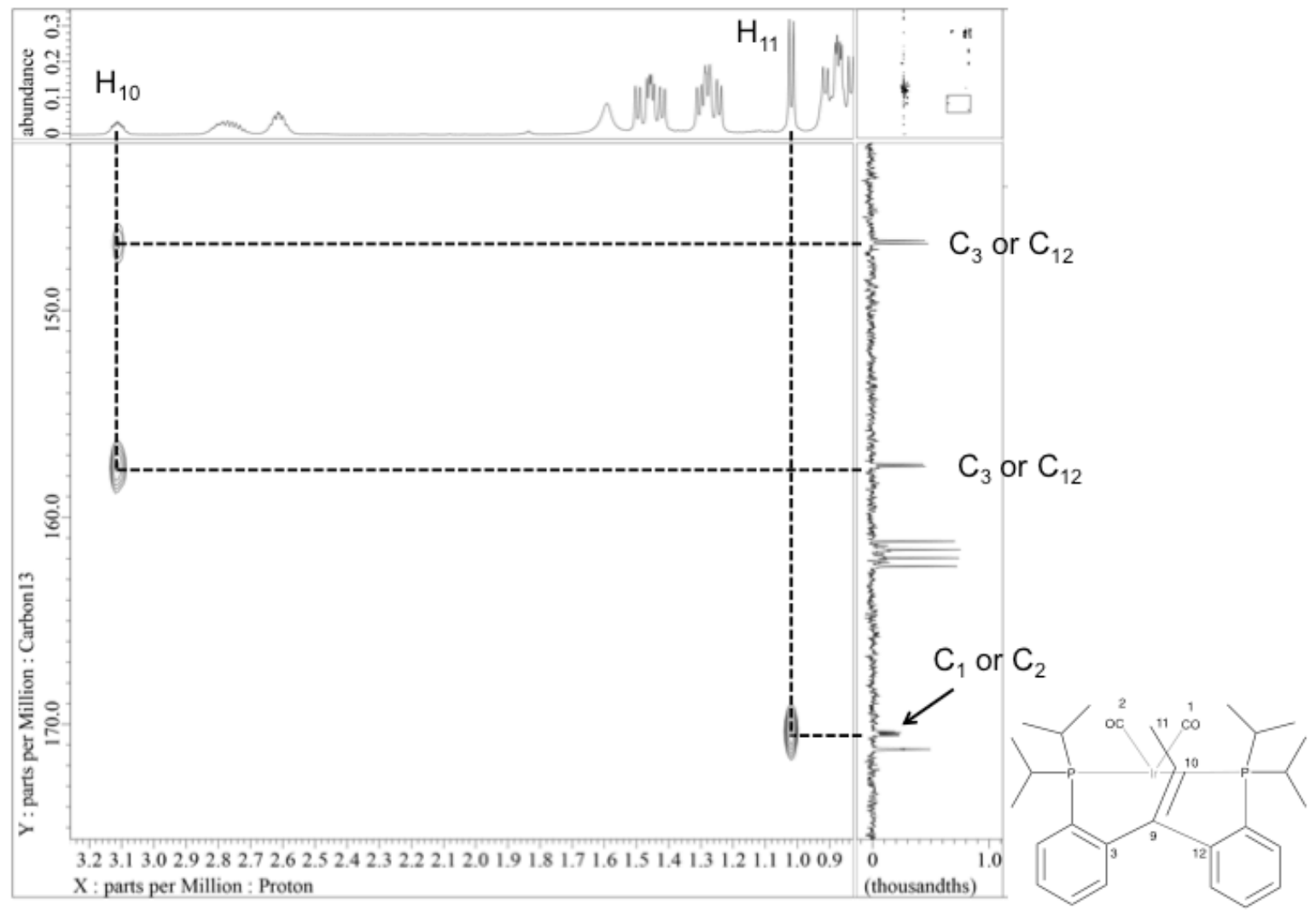

Figure S61. Selected region-2 of HMBC spectrum of Ir'-C 2_alkene

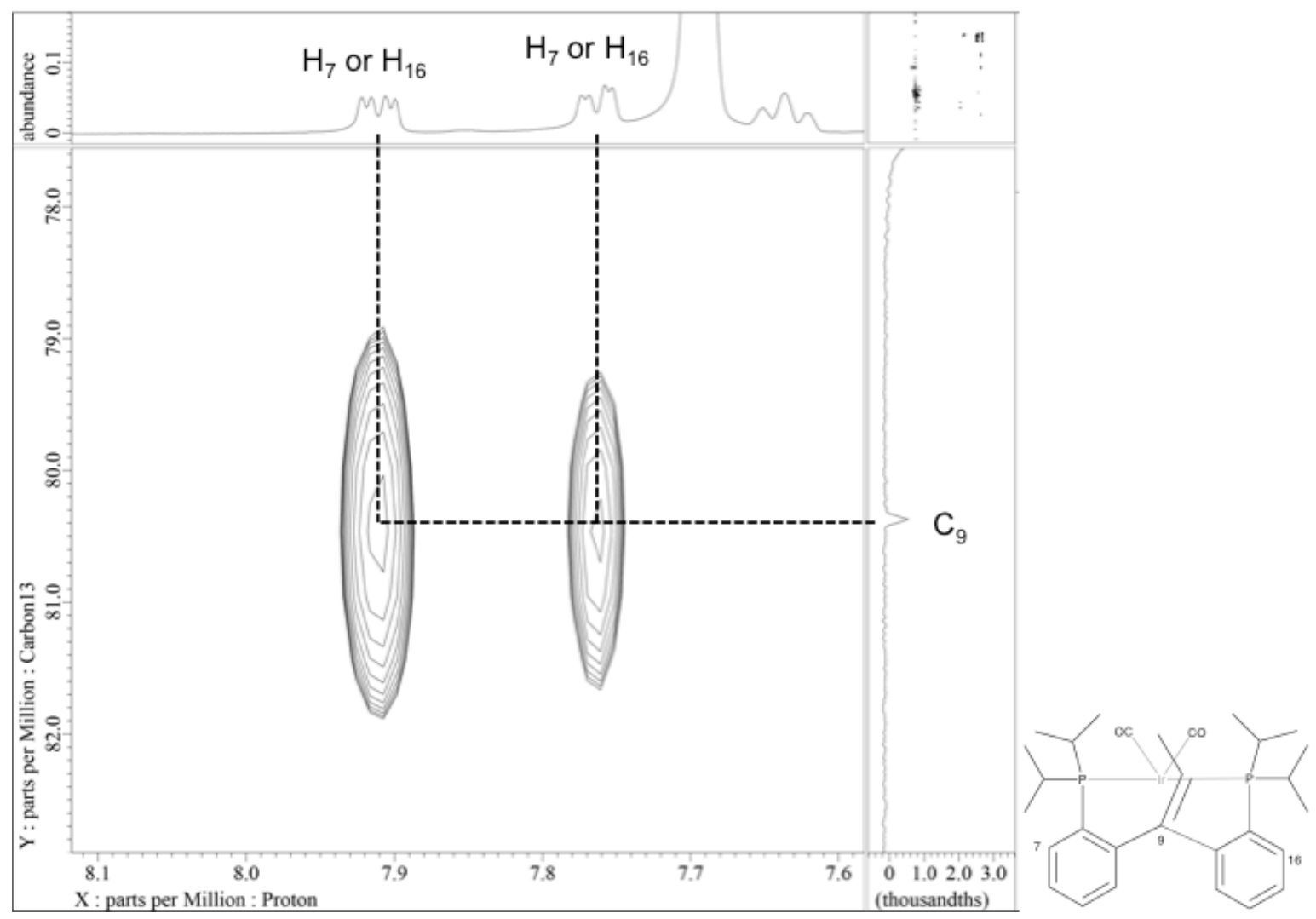

Figure S62. Selected region-3 of HMBC spectrum of Ir'-C $\mathbf{2}_{2}$ alkene 


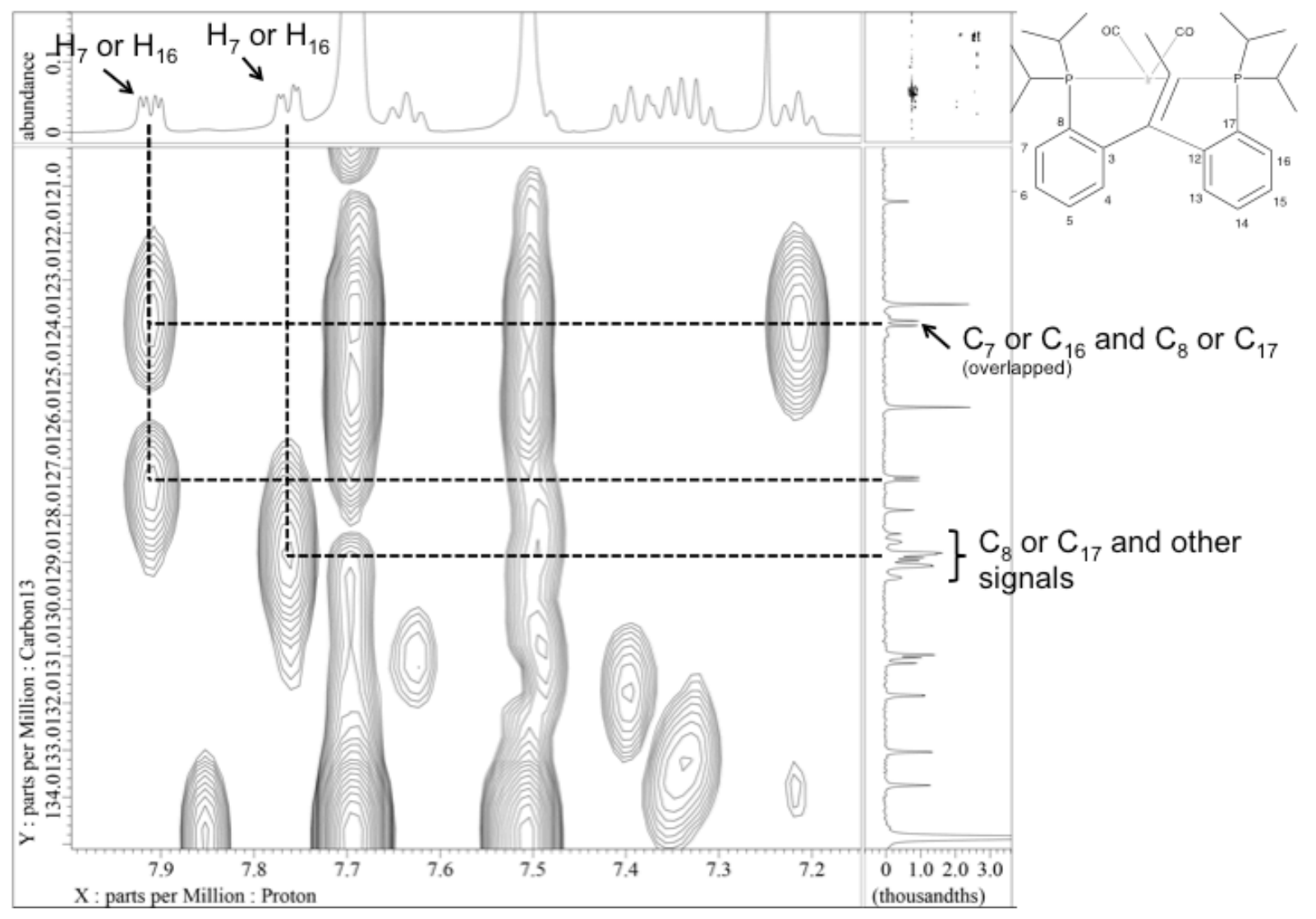

Figure S63. Selected region-4 of HMBC spectrum of Ir'-C 2_alkene

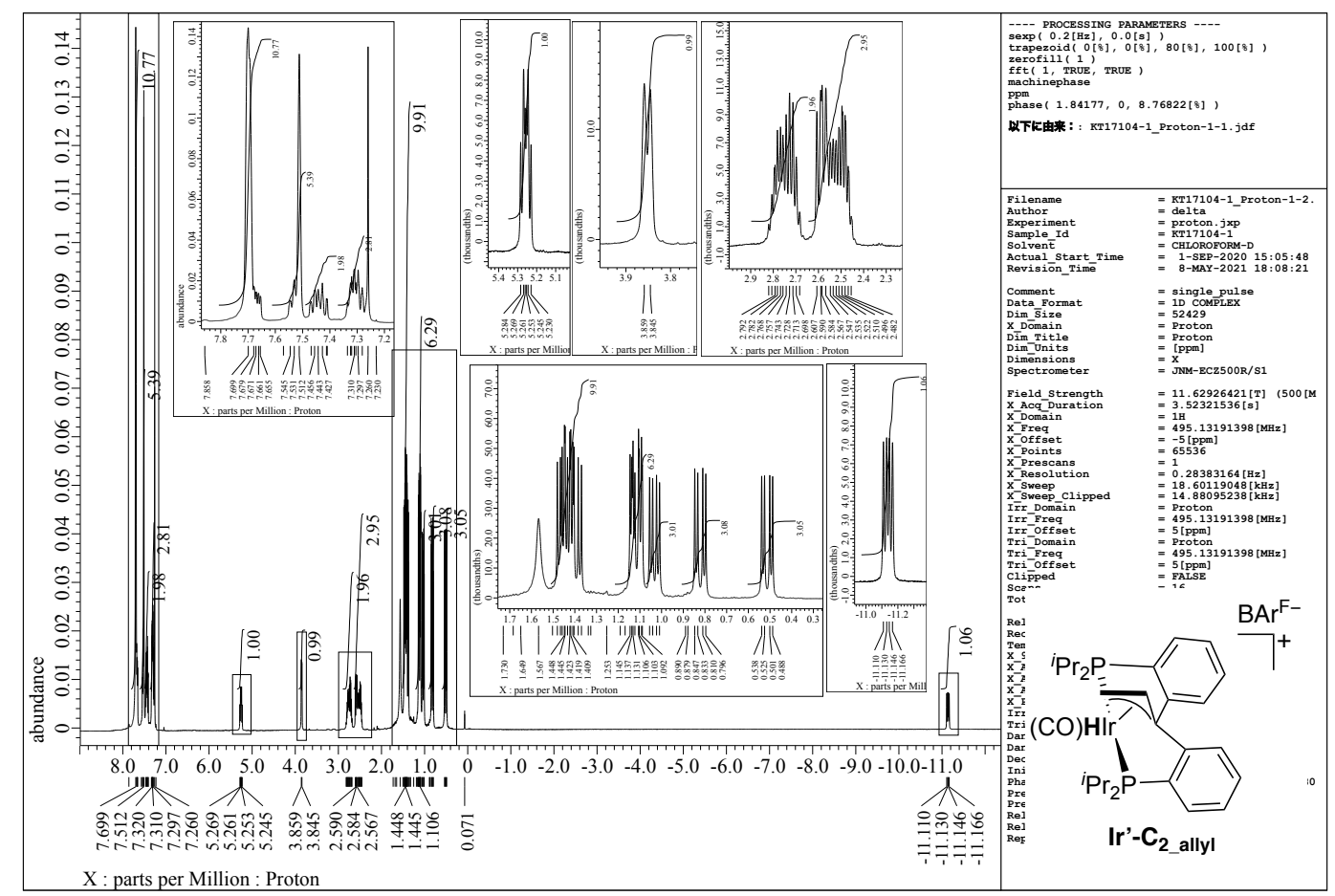

Figure S64. ${ }^{1} \mathrm{H}$ NMR spectrum of Ir'-C 2_allyl 


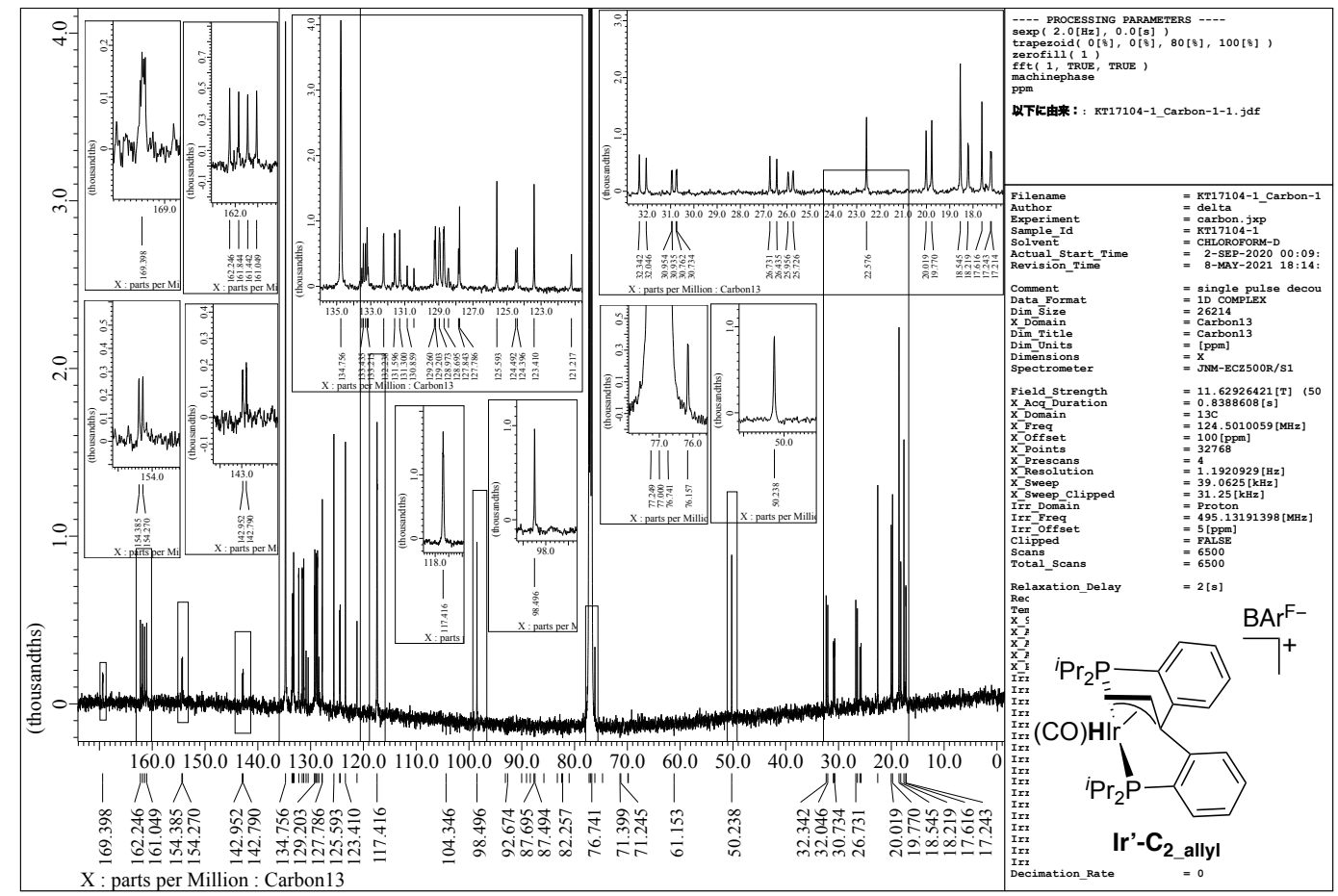

Figure S65. ${ }^{13} \mathrm{C}$ NMR spectrum of Ir'-C $\mathbf{2}_{2}$ allyl

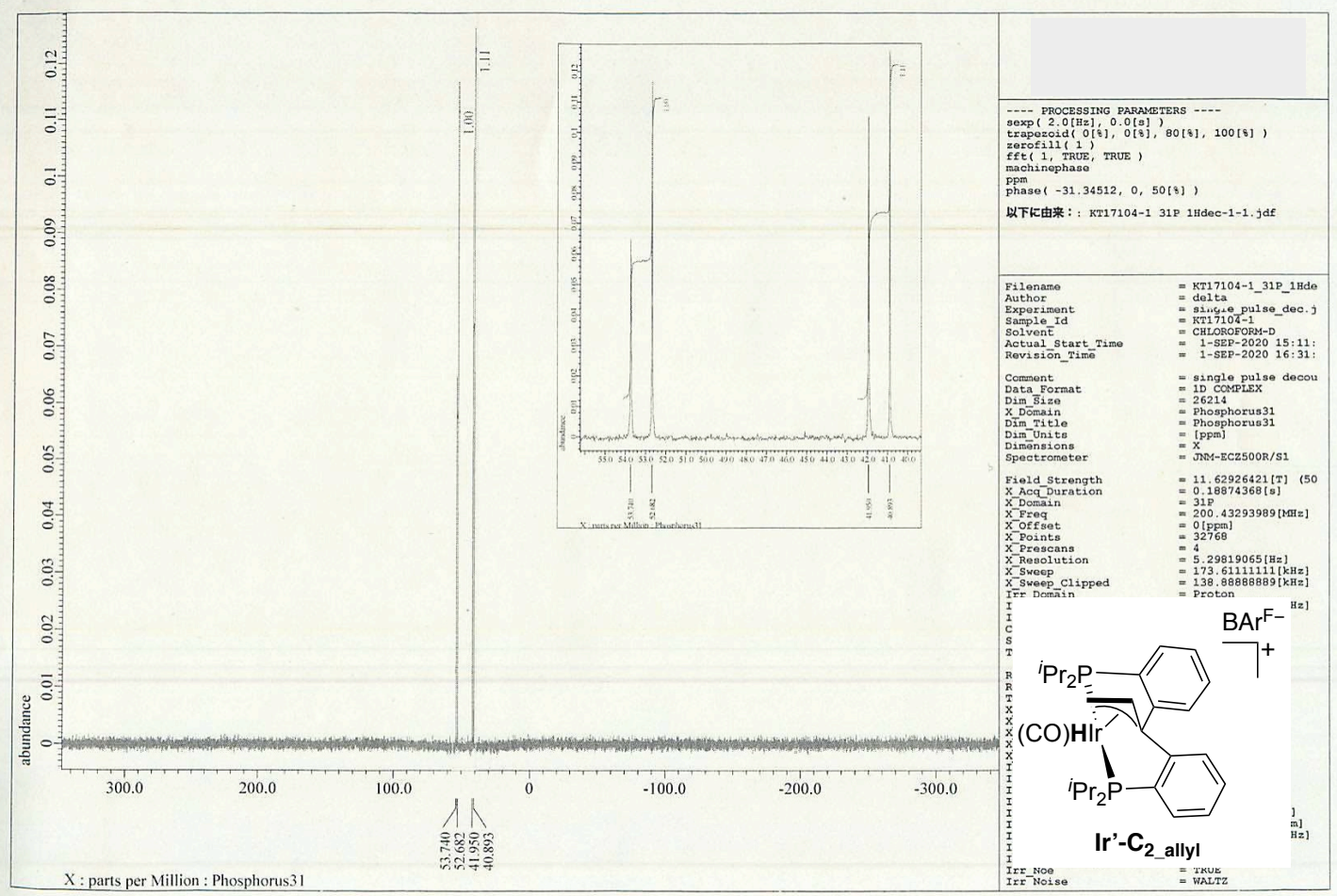

Figure S66. ${ }^{31} \mathrm{P}$ NMR spectrum of Ir'-C $\mathbf{C}_{\mathbf{2}}$ allyl 


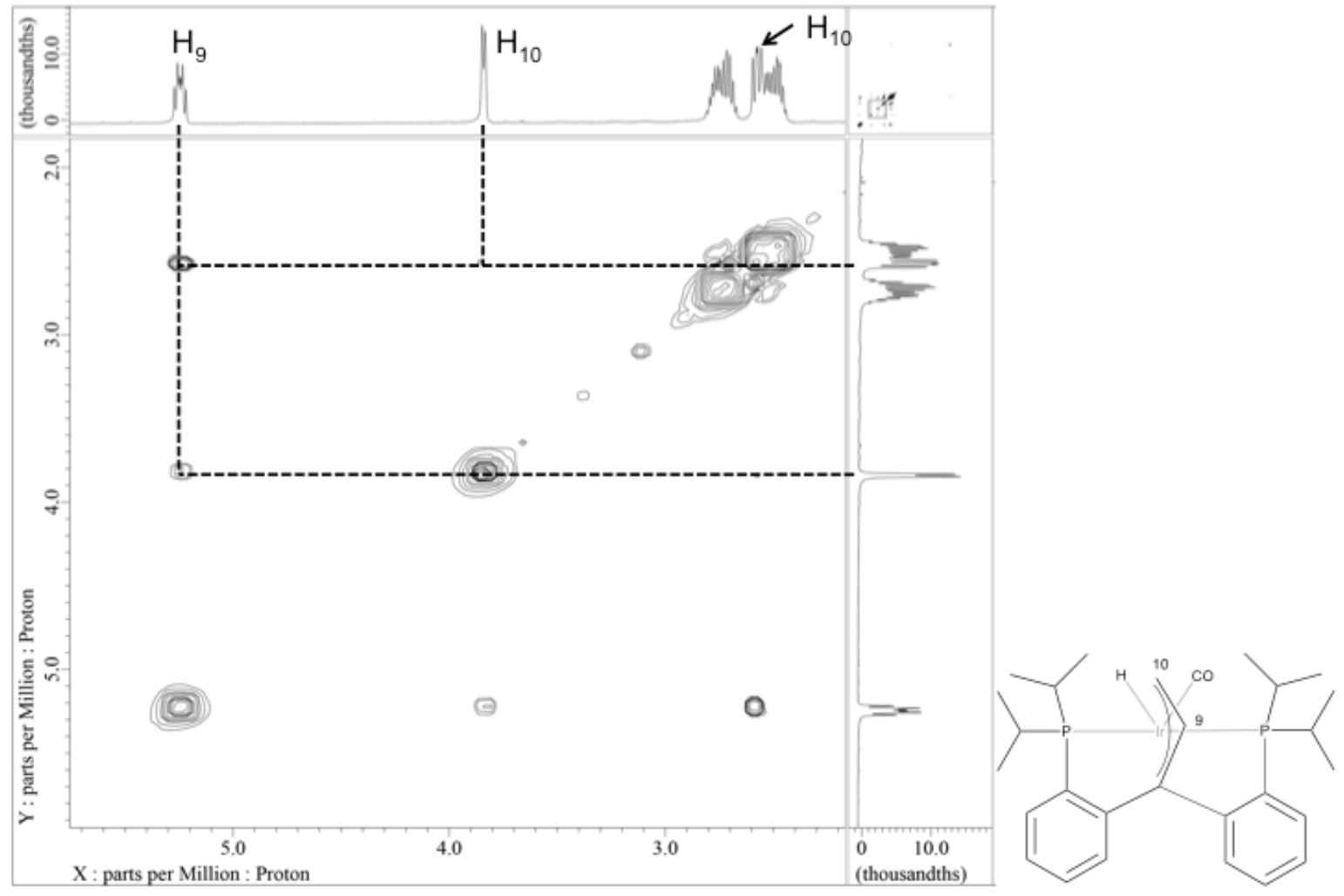

Figure S67. Selected region-1 of HHCOSY spectrum of Ir'-C $\mathbf{2}_{2}$ allyl

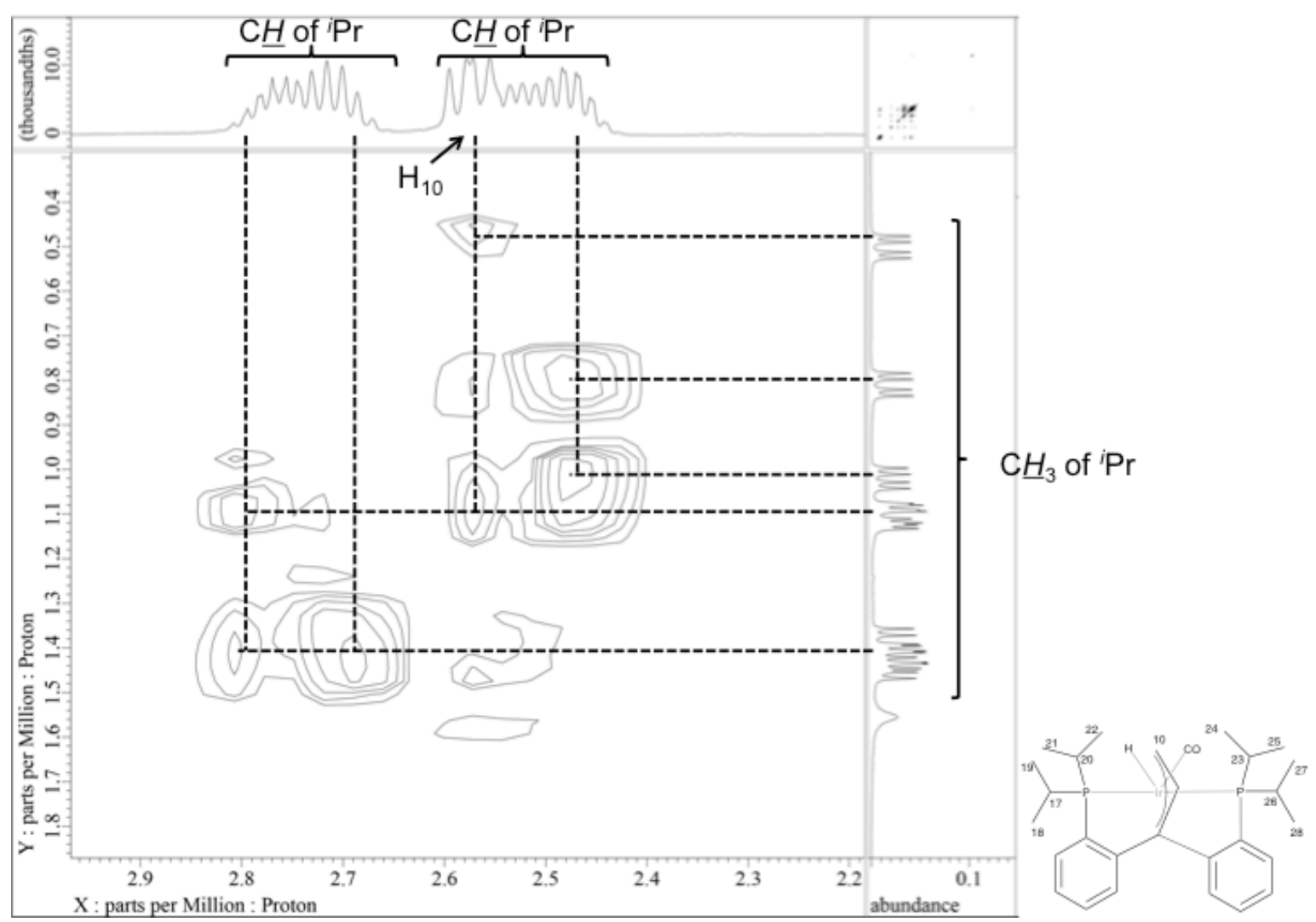

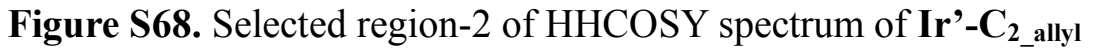




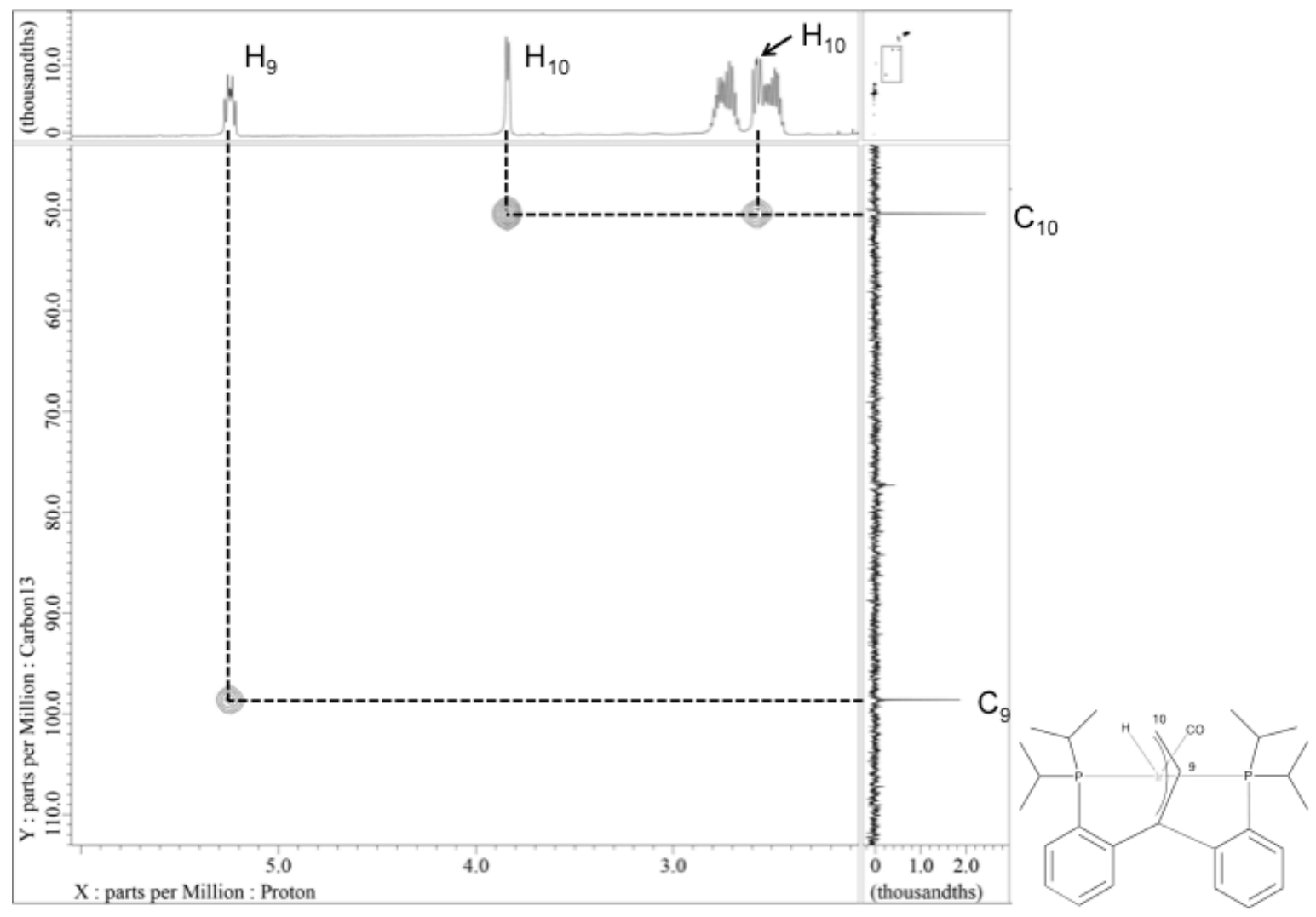

Figure S69. Selected region of HMQC spectrum of Ir'-- $\mathbf{C}_{2}$ allyl

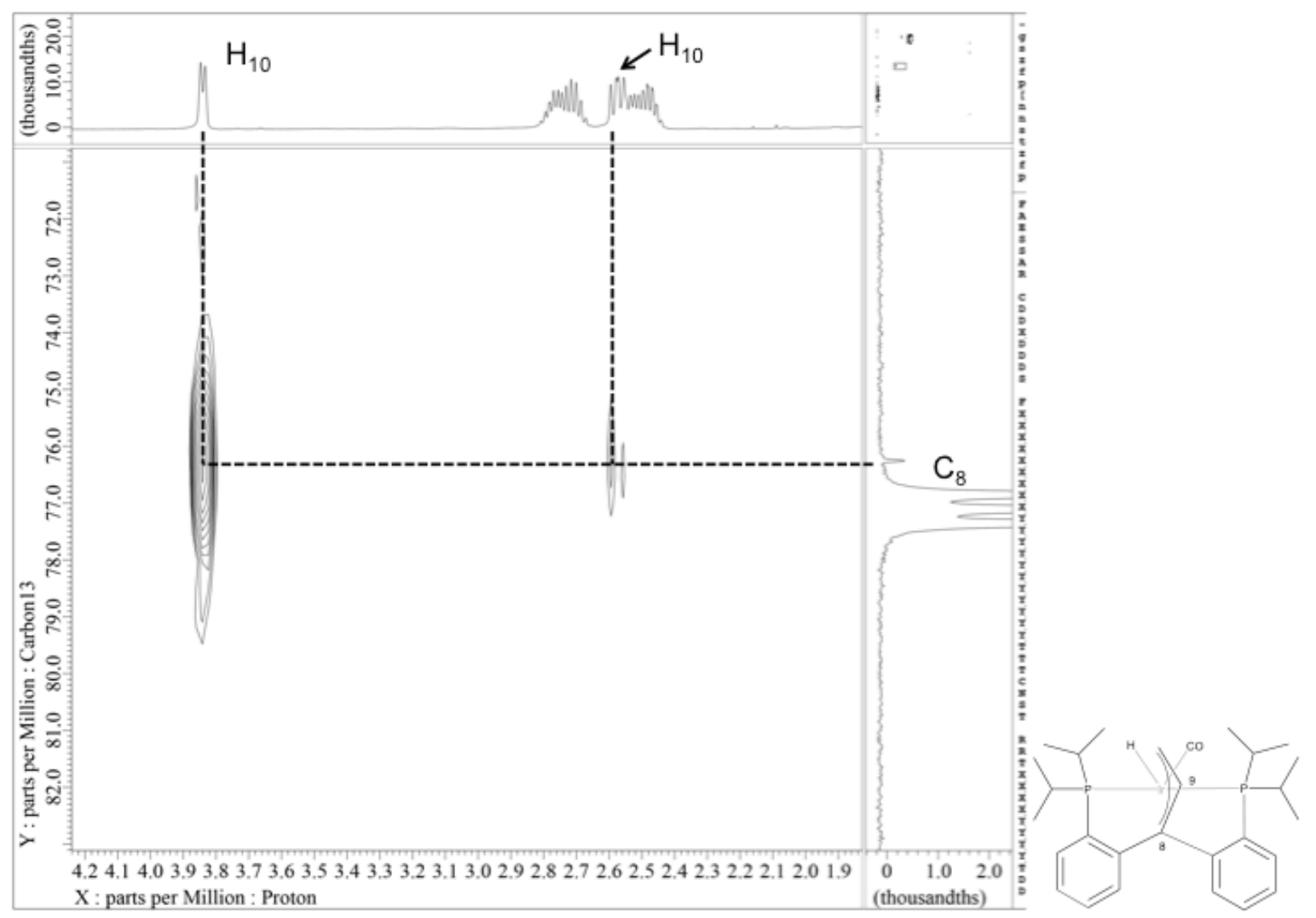

Figure S70. Selected region-2 of HMBC spectrum of Ir'-C $\mathbf{2}_{2}$ allyl 


\section{References}

1) Generation of Alkoxyl Radicals by Photoredox Catalysis Enables Selective C( $\left(\mathrm{sp}^{3}\right)-\mathrm{H}$ Functionalization under Mild Reaction Conditions. Zang, J.; Li, Y.; Zhang, F.; Hu, C.; Chen, Y. Angew. Chem. Int. Ed. 2016, 55, 1872-1875. 2) Phosphine- and Amine-Borane Dehydrocoupling Using a Three Coordinate Iron(II) $\beta$-Diketiminate Precatalyst. Coles, N. T.; Mahon, M. F.; Webster, R. L. Organometallics 2017, 36, 2262-2268.

3) Frisch, M. J.; Trucks, G. W.; Schlegel, H. B.; Scuseria, G. E.; Robb, M. A.; Cheeseman, J. R.; Scalmani, G.; Barone, V.; Petersson, G. A.; Nakatsuji, H.; Li, X.; Caricato, M.; Marenich, A. V.; Bloino, J.; Janesko, B. G.; Gomperts, R.; Mennucci, B.; Hratchian, H. P.; Ortiz, J. V.; Izmaylov, A. F.; Sonnenberg, J. L.; Williams-Young, D.; Ding, F.; Lipparini, F.; Egidi, F.; Goings, J.; Peng, B.; Petrone, A.; Henderson, T.; Ranasinghe, D.; Zakrzewski, V. G.; Gao, J.; Rega, N.; Zheng, G.; Liang, W.; Hada, M.; Ehara, M.; Toyota, K.; Fukuda, R.; Hasegawa, J.; Ishida, M.; Nakajima, T.; Honda, Y.; Kitao, O.; Nakai, H.; Vreven, T.; Throssell, K.; Montgomery, J. A., Jr.; Peralta, J. E.; Ogliaro, F.; Bearpark, M. J.; Heyd, J. J.; Brothers, E. N.; Kudin, K. N.; Staroverov, V. N.; Keith, T. A.; Kobayashi, R.; Normand, J.; Raghavachari, K.; Rendell, A. P.; Burant, J. C.; Iyengar, S. S.; Tomasi, J.; Cossi, M.; Millam, J. M.; Klene, M.; Adamo, C.; Cammi, R.; Ochterski, J. W.; Martin, R. L.; Morokuma, K.; Farkas, O.; Foresman, J. B.; Fox, D. J. Gaussian, Inc., Wallingford CT, 2016.

4) a) Density-functional thermochemistry. III. The role of exact exchange. Becke, A. D. J. Chem. Phys. 1993, 98 , 5648-5652. b) Development of the Colle-Salvetti correlation-energy formula into a functional of the electron density. Lee, C.; Yang, W.; Parr, R. G. Phys. Rev. B 1988, 37, 785-789.

5) Ab initio effective core potentials for molecular calculations. Potentials for $\mathrm{K}$ to Au including the outermost core orbitals. Hau, P. J.; Wadt, W. R. J. J. Chem. Phys. 1985, 82, 299-310.

6) Self-Consistent Molecular-Orbital Methods. IX. An Extended Gaussian-Type Basis for Molecular-Orbital Studies of Organic Molecules. J. Chem. Phys. 1971, 54, 724-728.

7) Cationic mono and dicarbonyl pincer complexes of rhodium and iridium to assess the donor properties of PC $_{\text {carbene }}$ P ligands. Smith, J. D.; Logan, J. R.; Doyle, L. E.; Burford, R. J.; Sugawara, S.; Ohnita, C.; Yamamoto, Y.; Piers, W. E.; Spasyuk, D. M.; Borau-Garcia, J. Dalton Trans. 2016, 45, 12669-12679. 